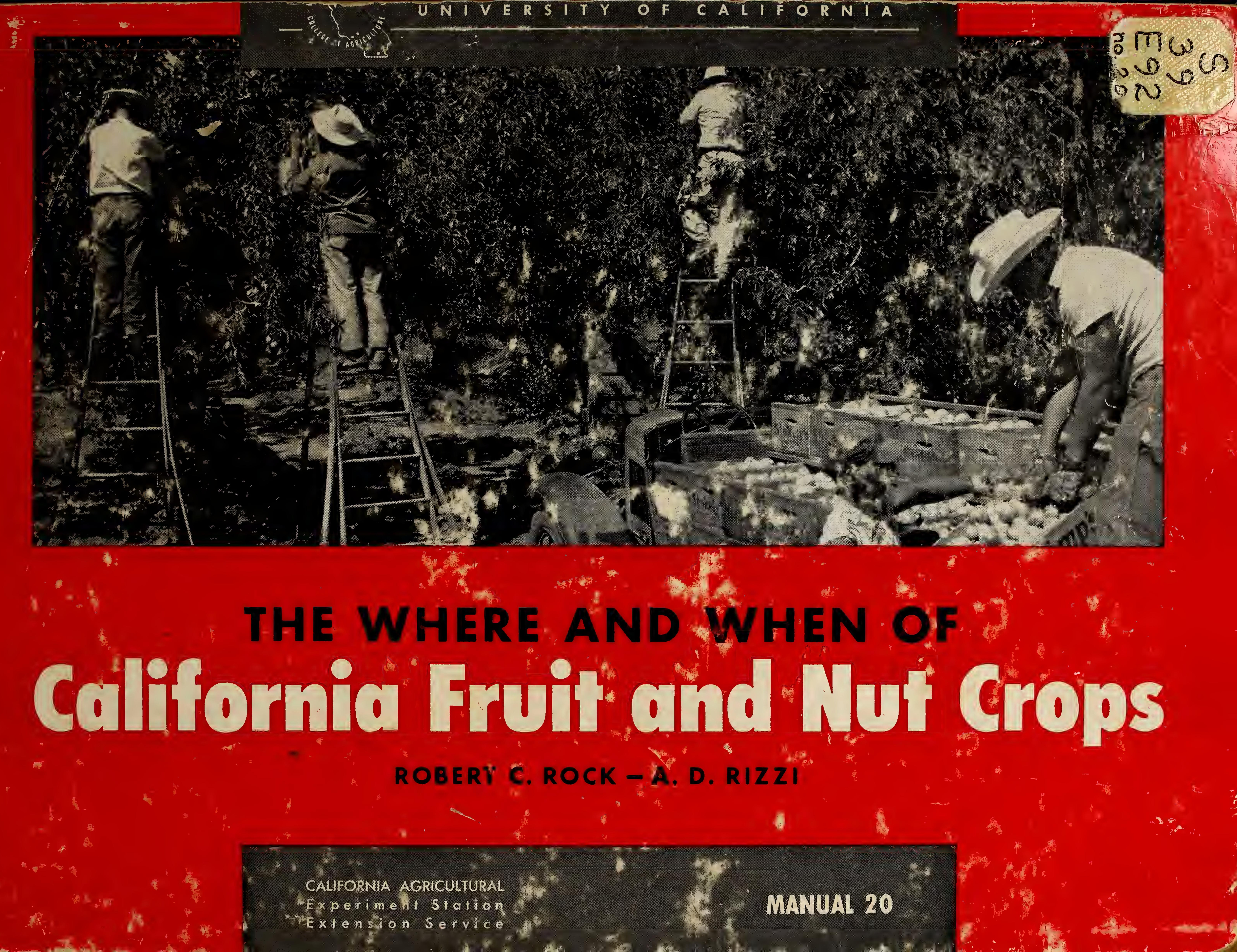




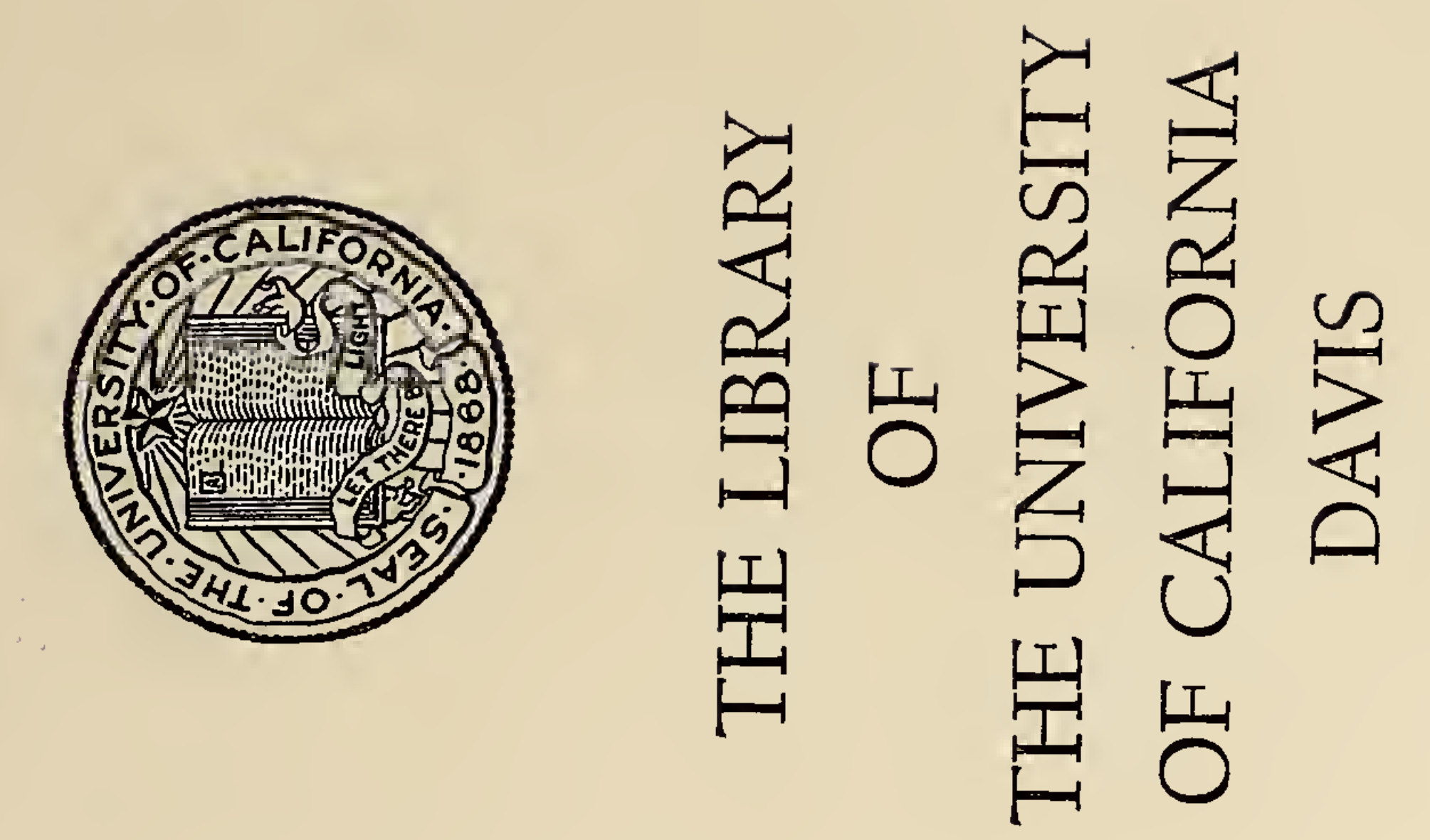




\section{THE WHERE AND WHEN OF California Fruit and Nut Crops}

ROBERT C. ROCK • A. D. RIZZI

\section{O}

\begin{tabular}{|c|c|c|c|}
\hline \multicolumn{3}{|c|}{ MAP } & MAP \\
\hline All fruits and nuts & 1 & Lemons .. & .. 11 \\
\hline Almonds ....... & 2 & Nectarines & 12 \\
\hline Apples .. & 3 & Olives ... & 13 \\
\hline Apricots . & 4 & Oranges $\ldots \ldots \ldots$ & 14 \\
\hline Avocados & 5 & Peaches (Clingstone) & 15 \\
\hline Cherries & 6 & Peaches (Freestone) & 16 \\
\hline Dates .. & 7 & Pears $\ldots \ldots \ldots \ldots$ & 17 \\
\hline Figs... . & 8 & Plums & 18 \\
\hline Grapefruit & 9 & Prunes. & 19 \\
\hline Grapes .. & 10 & Walnuts & 20 \\
\hline
\end{tabular}

A series of maps and calendars showing the prodvation areas of important fruit and nut crops grown in Colltornia and the usual harvest seasons for each crop.
THIS MANUAL is one of a series published by the University of California College of Agriculture and sold for a charge which is based upon returning only a portion of the production cost. By this means it is possible to make available publications which, due to relatively high cost of production, or limited audience, would otherwise be beyond the scope of the College publishing program.

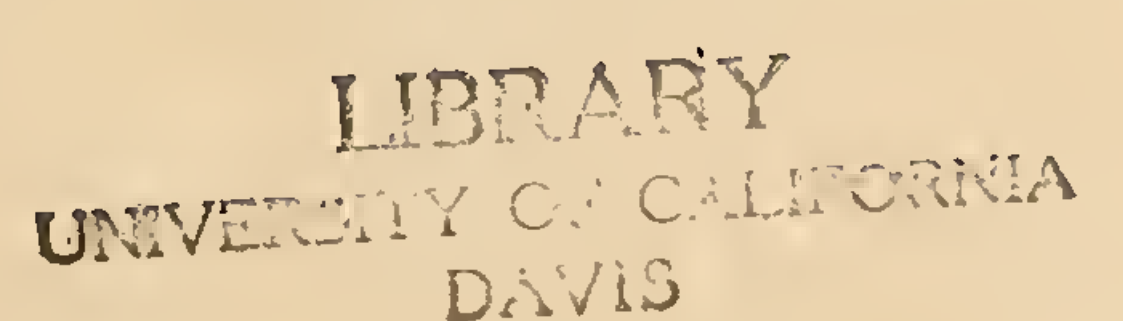




\section{WHERE}

Important production areas are outlined in color on a separate map for each crop. Areas are outlined on the basis of differences in location, varietal composition, and harvest seasons.

Major concentrations of plantings are shown in solid red. Minor concentrations and scattered plantings are indieated by small red dots. While acreage is an indicator of production for a particular area, the relative production between areas may vary because of differences in yield per acre.

Statistical information on varietal composition of each area, and data on state acreage are given on the reverse of each map.

\section{WHEN}

Harvesting periods are indicated on separate calendars for each crop. Each bar on the calendar shows total period of harvest (length of bar) and peak period of harvest (solid section of bar).

All periods shown are usual harvest times. Actual harvest periods may vary considerably from year to year as a result of: 1) changing weather conditions; 2) market and economic conditions; 3) availability of harvest labor; 4) perishability of the crop; 5 ) development of new mechanical harvesting equipment and techniques; and 6) cultural practices and conditions, such as irrigation, fertilizer programs, age of trees, and the like.

\section{THE AUTHORS:}

Robert C. Rock is Associate Agriculturist (Marketing) and Associate on the Giannini Foundation, Riverside.

A. D. Rizzi is Agriculturist (Pomology), Davis.

\section{ACKNOWLEDGMENTS}

The authors wish to express their appreciation to the following individuals and organizations who have helped in the preparation of this manual: Mr. George A. Scott and Mr. R. E. Blair of the California Crop and Livestock Reporting Service, Sacramento; members of the departments of Pomology and Viticulture at Davis; University of California Farm Advisors and Specialists in the Extension Service; County Agricultural Commissioners; and many individual growers and industry associations. 


\section{PRODUCTION AREAS}

AREA 1: 9 per cent of the store's totol ocreoge, Counties: Sulter, Butte, AREA 2: 11 per cent ol the stote's totol ocreoge. Counties: Son Jooquin, Yolo, Coluso, Teliomo, Glenn, Yubo, ond Shosio (Socromento Valley). Coniro Costo, Solono, and Sacromento (Delto ond odjocent oreo). Prin. Principol lruit ond nut cropsiolmonds, olives, cipal fruil ond nut crops: olmonds, opricols, cherrles, gropes, peaches, peors, prunes, ond wolnus.

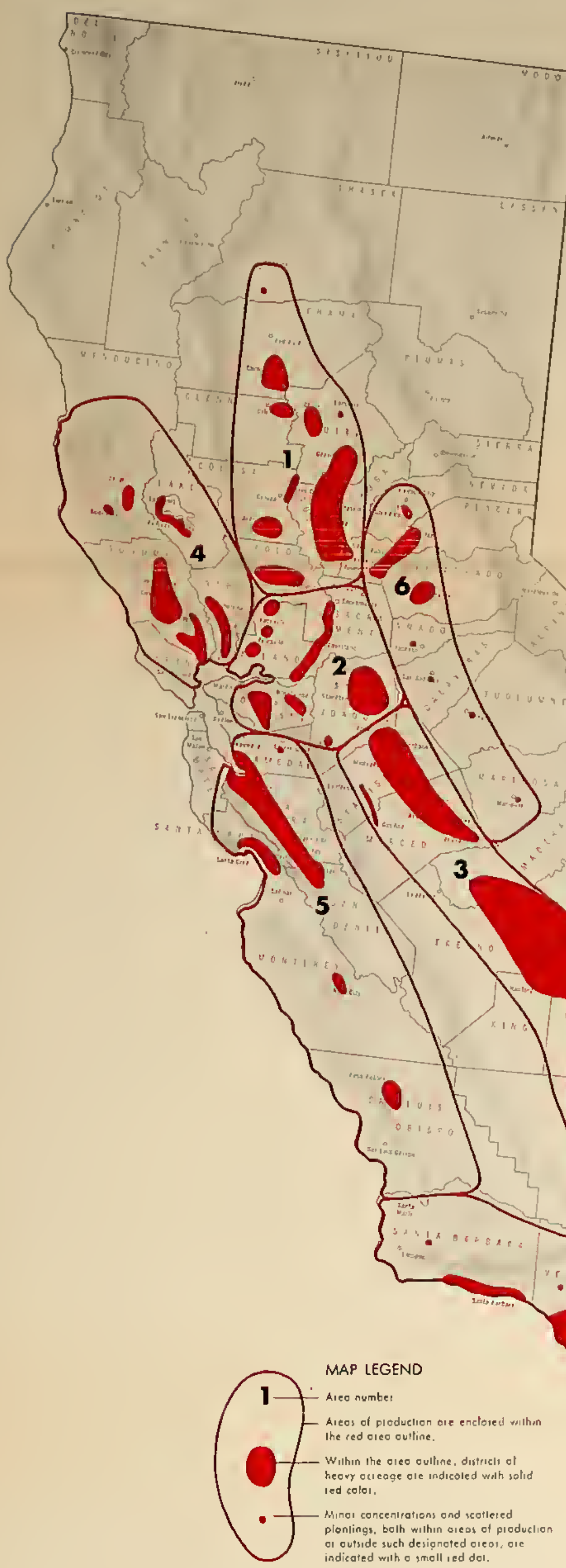

AREA 3: 36 per cent ol the store's totol ocreage. Counties: Fresno, Tulore, cipol lruit and nut crops, olmonds, figs, aropes, lemons, nectorines, dives, oronges, peoches, plums, ond wolnuts.

AREA 4: 6 per cent of the stote's totol ocreoge. Countles: Sonomo, Napo, loke, Mendocino, ond Marin (North Cooss). Principol lruit ond nut crops: opples, cherries, gropes, peors, prunes, ond wainuts.

AREA 5: 10 per cent of the stote's totol ocreage. Counties: Sonto Clora, Sonto CCuz, Son Bentio, Son luis Obispo, Alomedo, Monterey, and san

AREA 6: 2 per cent of the state's totol ocreoge. Counties: Plocer, El Dorodo, Nevodo, Coloveros, Amodor, Tuolumne, and Moriposo (Sierro Nevodo foothillst. Principol truit ond nut crops: opples, peors, ond plums.

AREA 7: 24 per cent of the stote's totol ocreoge. Counties: San Bernor. dino, Venturo, Oronge, Los Angeles, Riverside Iwestern), Son Diego (west. opples, opricors, ovocodos, gropetruit, gropes, lemans, oronges, peoches, opples, opricars,
ond wolnuts.

AREA 8: 2 per cent of the stote's lotol ocreoge. Counties: Riverside leostern', Impariat, and Son Diego leastorn).
ond nut crops: doles, gropelfuit, ond gropes.

\section{USUAL HARVEST SEASONS}

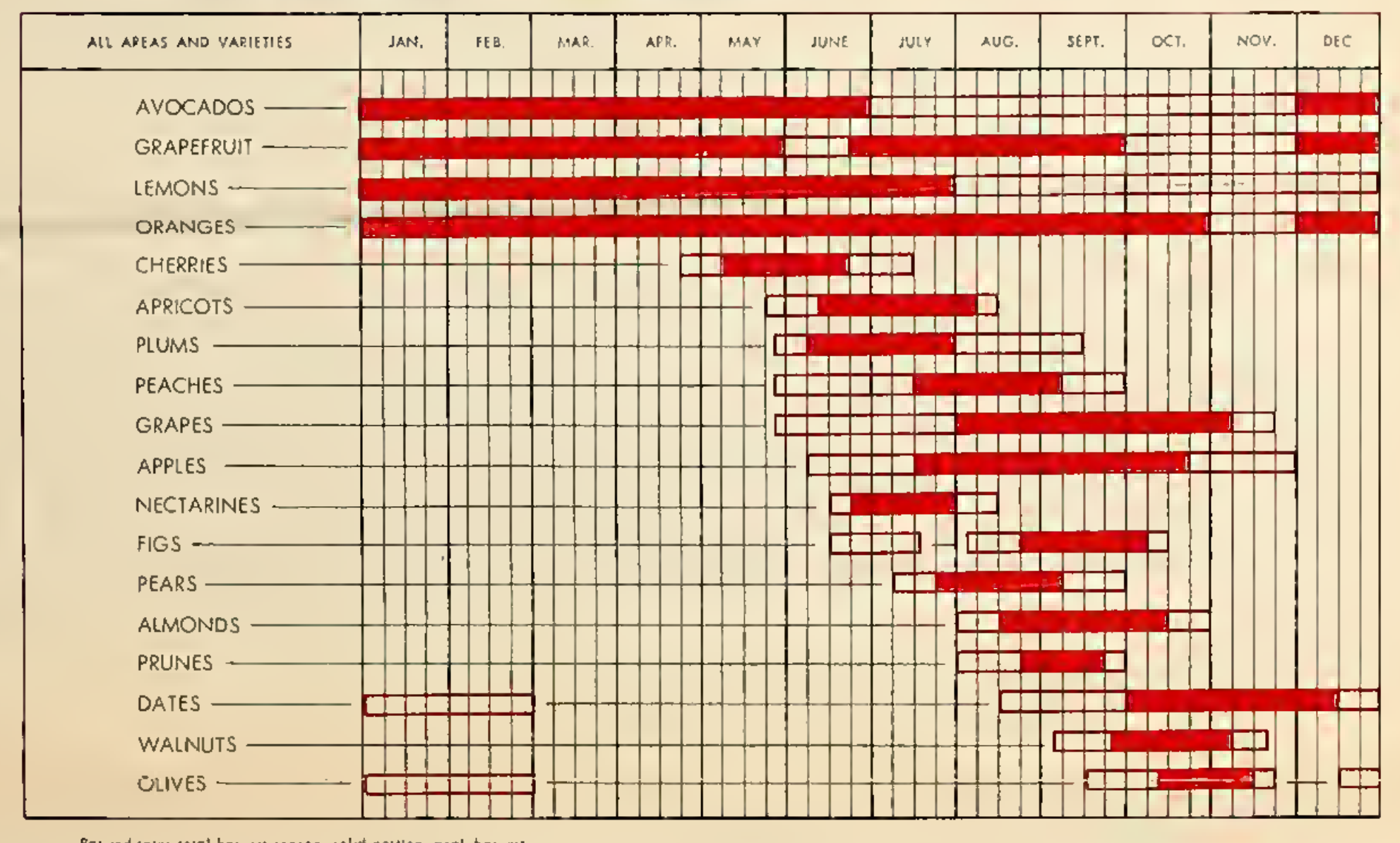

FRUIT AND NUT CROPS_Mop 1 of Monual 20.
"The Where ond When of Colifornio Frvit and Nut 


\begin{tabular}{|c|c|c|c|}
\hline \multicolumn{4}{|c|}{$\begin{array}{l}\text { Tablo } 1 \text { (Map I)-All Californla Fruir and Nut Cropsi* Total Acroago } \\
\text { (Boaring and Nonbearing) by Areas and Countles, } 1953\end{array}$} \\
\hline Ares and eounisy & $\begin{array}{l}\text { Number of } \\
\text { actes } \\
\text { ats }\end{array}$ & Ares and coonly & $\begin{array}{l}\text { Numbere ol } \\
\text { sures } \\
\text { ses }\end{array}$ \\
\hline $\begin{array}{l}\text { Area 1: } \\
\text { Butte.. }\end{array}$ & & $\begin{array}{l}\text { Area 5: } \\
\text { Alammedn. }\end{array}$ & \\
\hline colusa. & 16,111 & $\begin{array}{l}\text { Aameda... } \\
\text { Monterey... }\end{array}$ & $\begin{array}{l}7,729 \\
6,252\end{array}$ \\
\hline Glenn... & 8,817 & San Benito........ & 12,011 \\
\hline $\begin{array}{l}\text { Shasta.. } \\
\text { Suttor }\end{array}$ & $\begin{array}{r}1,228 \\
{ }_{3418}\end{array}$ & San Lutis Obispo. & 10,996 \\
\hline Sutter... & $\begin{array}{l}34,418 \\
11247\end{array}$ & $\begin{array}{l}\text { San Mateo.. } \\
\text { Sants Clasa }\end{array}$ & $\begin{array}{r}2999 \\
84.936\end{array}$ \\
\hline $\begin{array}{l}\text { Tebama. } \\
\text { Yolo.... }\end{array}$ & $\begin{array}{l}11,1247 \\
15,505\end{array}$ & $\begin{array}{l}\text { Santa CCara } \\
\text { Santa Cruz.. }\end{array}$ & $\begin{array}{l}\begin{array}{l}84,936 \\
13,271\end{array} \\
\end{array}$ \\
\hline Yaba.. & & Area total. & \multirow{2}{*}{134,694} \\
\hline Area total. & 129,141 & $A-c_{0}$ & \\
\hline Area 2: & & Amador... & 1,138 \\
\hline Contra Costa. & $\begin{array}{l}33,888 \\
11733\end{array}$ & $\begin{array}{l}\text { Calaveras. } \\
\text { El Dorado. }\end{array}$ & 1,256 \\
\hline $\begin{array}{l}\text { Sacramento... } \\
\text { Sen Josonin . }\end{array}$ & $\begin{array}{l}11,733 \\
97,069\end{array}$ & $\begin{array}{l}\text { Midorado. } \\
\text { Mariposa }\end{array}$ & $\begin{aligned} 4,903 \\
432\end{aligned}$ \\
\hline \multirow[t]{2}{*}{$\begin{array}{l}\text { San Joaquin. } \\
\text { Solang....... }\end{array}$} & $\begin{array}{l}98,821 \\
18,269\end{array}$ & Nevada. . . & \multirow{2}{*}{$\begin{array}{r}1,329 \\
18,393 \\
679\end{array}$} \\
\hline & 161,551 & $\begin{array}{l}\text { Pacel..... } \\
\text { Tuolumne. }\end{array}$ & \\
\hline Area 3: & & Area total. & \multirow{2}{*}{28,130} \\
\hline Fresno.. & 202,739 & & \\
\hline & $\begin{array}{r}30,660 \\
9,205\end{array}$ & \multirow{5}{*}{ 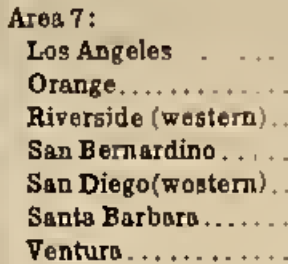 } & \\
\hline Madera.. & 23,053 & & \\
\hline Merced... & $\begin{array}{l}43,101 \\
58361\end{array}$ & & $\begin{array}{l}62,010 \\
37,027\end{array}$ \\
\hline $\begin{array}{l}\text { Stassislaus... } \\
\text { Tulare ..... }\end{array}$ & $\begin{array}{r}54,361 \\
140,021\end{array}$ & & $\begin{array}{l}37,027 \\
74,814\end{array}$ \\
\hline Area total. . & $\overline{507,140}$ & & $\begin{array}{l}30,371 \\
14400 \\
63,868\end{array}$ \\
\hline \multirow{2}{*}{$\begin{array}{l}\text { Area 4: } \\
\text { A takese }\end{array}$} & & Area total.... & \multirow{2}{*}{$\begin{array}{l}337,355 \\
\end{array}$} \\
\hline & 11,573 & & \\
\hline \multirow{3}{*}{$\begin{array}{l}\text { Marin....... } \\
\text { Mendocino.. } \\
\text { Napa....... } \\
\text { Sonoma.... }\end{array}$} & $\begin{array}{r}434 \\
11,459\end{array}$ & \multirow{4}{*}{$\begin{array}{l}\text { Area 8: } \\
\text { Imperial ........ } \\
\text { Rivergide (easterm) } \\
\text { San Diego (eastern) } \\
\text { Area total ....... }\end{array}$} & \multirow{2}{*}{$\begin{array}{r}3,601 \\
17,152\end{array}$} \\
\hline & 22,599 & & \\
\hline & 42,679 & & 1,610 \\
\hline \multirow[t]{3}{*}{ Area total. } & 88,644 & & 22,263 \\
\hline & & Additional counties .. & 618 \\
\hline & & State total .... & $1,409,536$ \\
\hline
\end{tabular}

\begin{tabular}{|c|c|c|c|}
\hline \multicolumn{4}{|c|}{$\begin{array}{l}\text { Table } 2 \text { (Map I)-All California fruits and Nuts: Total Acreage } \\
\text { (Bearlng and Nonbearing) by Commodlflos, } 1953\end{array}$} \\
\hline Commodity & 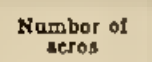 & Commodits & $\begin{array}{c}\text { Nambor of } \\
\text { actres }\end{array}$ \\
\hline Almonds. & 102,470 & Nectarines.. & 4,310 \\
\hline Apples... & 26,220 & Olipes...... & 30,622 \\
\hline Apricots... & 44,012 & Oranges. & 197,261 \\
\hline Arocados. & 22,106 & Peacbes. & 95,651 \\
\hline Cherries... & 11,284 & Pears... & 42,973 \\
\hline $\begin{array}{l}\text { Chestnuts. } \\
\text { Dates...... }\end{array}$ & $\begin{array}{r}257 \\
5586 \\
5\end{array}$ & $\begin{array}{l}\text { Pecans....... } \\
\text { Pergimmons. }\end{array}$ & $\begin{array}{l}635 \\
698\end{array}$ \\
\hline $\begin{array}{l}\text { Daigs....... } \\
\text { Figs.... }\end{array}$ & $\begin{array}{l}0,0,666 \\
26,665\end{array}$ & $\begin{array}{l}\text { Perrimimmons... } \\
\text { Plums....... }\end{array}$ & $\begin{array}{r}698 \\
25,120\end{array}$ \\
\hline Filherts.... & ${ }_{39}$ & Pomegranates. & $\begin{array}{l}20,120 \\
546\end{array}$ \\
\hline Grapefruit. & $\begin{array}{r}9,718 \\
\end{array}$ & Prunes......... & 101,005 \\
\hline \multirow{3}{*}{$\begin{array}{l}\text { Grapes.. } \\
\text { Lemons.. } \\
\text { Limes.... }\end{array}$} & 464,031 & Quinces. & \\
\hline & 60,745 & Walnuts. & 137,614 \\
\hline & & State total. & $1,409,536$ \\
\hline
\end{tabular}

\begin{tabular}{|c|c|c|c|c|c|c|c|}
\hline \multicolumn{8}{|c|}{ Table 3 (Map 1)-Climatalogical Information far Selected Stations in Caijfornia } \\
\hline \multirow{2}{*}{ Areas and ulation } & \multirow{2}{*}{ sloration } & \multirow{2}{*}{ 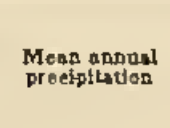 } & \multicolumn{5}{|c|}{ Temperature eammary } \\
\hline & & & Meten & $\begin{array}{c}\text { Mean } \\
\text { dally max. }\end{array}$ & 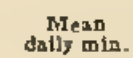 & Higkest & Lover: \\
\hline Area 1 -Sacramento Valley: & ieet & Inches & degreess & defrees & degrees & degreos & derseo. \\
\hline Colusa .................... & 60 & 15.95 & 60.9 & 74.1 & 47.8 & & \\
\hline Marysville. & 65 & 19.77 & 62.6 & 75.0 & 48.1 & 118 & 16 \\
\hline Orovilie... & 272 & 27.34 & 61.9 & 75.7 & 47.5 & 119 & 13 \\
\hline Red Bluffi & & & & 73.8 & 50.8 & & \\
\hline & & & & 74.2 & & & 17 \\
\hline \multicolumn{8}{|l|}{$\begin{array}{l}\text { Area } 2-\text { Delta and adjacent are as: } \\
\text { Sacramento }\end{array}$} \\
\hline Sacramento.................. & 25 & 18.05 & & 71.7 & & 114 & 17 \\
\hline $\begin{array}{l}\text { Sttockton. } \\
\text { Winters. }\end{array}$ & 15 & 14.11 & 60.1 & 72.1 & 47.9 & 110 & 18 \\
\hline & & & & & & & 18 \\
\hline \multicolumn{8}{|l|}{$\begin{array}{l}\text { Area 3-San Joaquin Valley: } \\
\text { A }\end{array}$} \\
\hline $\begin{array}{l}\text { Bakersfield ............... } \\
\text { Fresno }\end{array}$ & 489 & 5.98 & 64.7 & $\begin{aligned} 79.0 \\
79,2\end{aligned}$ & & 118 & 13 \\
\hline $\begin{array}{l}\text { Fresno... } \\
\text { Merced. }\end{array}$ & 331 & $\begin{array}{r}9.41 \\
11.26\end{array}$ & $\begin{array}{l}63.3 \\
61.8\end{array}$ & $\begin{array}{l}76.3 \\
75.7\end{array}$ & 50.2 & 115 & 17 \\
\hline $\begin{array}{l}\text { Mercedd... } \\
\text { Modesto. }\end{array}$ & $\begin{array}{c}173 \\
91\end{array}$ & $\begin{array}{l}11.26 \\
10.99\end{array}$ & $\begin{array}{l}61.8 \\
60.1\end{array}$ & $\begin{array}{l}75.7 \\
73.9\end{array}$ & $\begin{array}{l}46.7 \\
46.4\end{array}$ & $\begin{array}{l}116 \\
111\end{array}$ & $\begin{array}{l}16 \\
15\end{array}$ \\
\hline Porterville. & 393 & $\begin{array}{l}10.99 \\
10.21\end{array}$ & $\begin{array}{l}60.1 \\
63.7\end{array}$ & $\begin{array}{l}73.9 \\
78.3\end{array}$ & $\begin{array}{l}46.9 \\
48.1\end{array}$ & ${ }_{114}^{111}$ & $\begin{array}{l}15 \\
18\end{array}$ \\
\hline \multicolumn{8}{|l|}{$\begin{array}{l}\text { Ares 4-North Coast: } \\
\text { Lakepor. }\end{array}$} \\
\hline Lakepor. ............. & 1,343 & 28.15 & 56.9 & 72.7 & 41.0 & 110 & 14 \\
\hline $\begin{array}{l}\text { Napa ......... } \\
\text { Sonta Ros. }\end{array}$ & $\begin{array}{r}60 \\
167\end{array}$ & $\begin{array}{l}23.44 \\
29.57\end{array}$ & $\begin{array}{c}57.8 \\
57.1\end{array}$ & $\begin{array}{l}70.4 \\
71.5\end{array}$ & 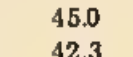 & 110 & 17 \\
\hline $\begin{array}{l}\text { Santa Rosa. } \\
\text { Wkiah...... }\end{array}$ & 650 & $\begin{array}{l}29.67 \\
35.35\end{array}$ & $\begin{array}{l}57.2 \\
57.8\end{array}$ & 73.8 & $\begin{array}{l}42.2 \\
42.2\end{array}$ & 114 & ${ }_{12}$ \\
\hline \multicolumn{8}{|l|}{ Area 5-Central Coast: } \\
\hline 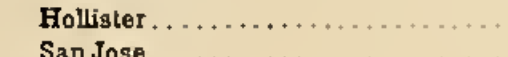 & 284 & 12.85 & 58.2 & 71.2 & 44.3 & 112 & 15 \\
\hline $\begin{array}{l}\text { San Jose............... } \\
\text { Paso Robles ....... }\end{array}$ & $\begin{array}{c}95 \\
740\end{array}$ & $\begin{array}{l}14.47 \\
15.67\end{array}$ & 58.3 & $\begin{array}{l}70.0 \\
76.1\end{array}$ & $\begin{array}{l}46.6 \\
40.7\end{array}$ & $\begin{array}{l}108 \\
117\end{array}$ & $\begin{array}{r}18 \\
0\end{array}$ \\
\hline \multicolumn{8}{|l|}{ Area 6-Sierra Nevada foothills: } \\
\hline 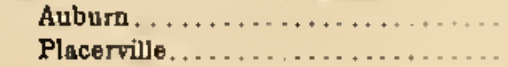 & $\begin{array}{l}1,234 \\
1,295\end{array}$ & $\begin{array}{l}33.47 \\
41.23\end{array}$ & $\begin{array}{l}60.3 \\
55.2\end{array}$ & $\begin{array}{l}73.2 \\
70.0\end{array}$ & $\begin{array}{l}{ }_{47.5}^{40.0} \\
40.0-30\end{array}$ & $\begin{array}{l}1122 \\
114\end{array}$ & $\begin{array}{r}12 \\
8\end{array}$ \\
\hline \multicolumn{8}{|c|}{ Area 7-Southern California (excluding desert area) : } \\
\hline & 660 & 16.76 & 61.3 & 76.0 & 46.3 & 113 & 13 \\
\hline $\begin{array}{l}\text { Pomona........ } \\
\text { Riverside ..... }\end{array}$ & $\begin{array}{l}855 \\
820\end{array}$ & $\begin{array}{l}18.40 \\
11.19\end{array}$ & $\begin{array}{l}61.1 \\
62.9\end{array}$ & $\begin{array}{l}77.0 \\
79.2\end{array}$ & $\begin{array}{l}45.1 \\
46.7\end{array}$ & $\begin{array}{l}117 \\
118\end{array}$ & ${ }_{19}^{22}$ \\
\hline San Fernando. & 950 & 16.09 & 62.9 & 77.7 & $\begin{array}{l}46.7 \\
48.2\end{array}$ & $\begin{array}{l}118 \\
113\end{array}$ & ${ }_{23}^{19}$ \\
\hline Sants Ana ... & ${ }^{133}$ & 13.85 & 62.1 & 75.7 & & 112 & 22 \\
\hline Santa Barbara. & 120 & $\begin{array}{ll}17.95 \\
15.6\end{array}$ & 59.9 & $\begin{array}{l}71.0 \\
74.6\end{array}$ & $\begin{array}{l}48.8 \\
45.5\end{array}$ & 115 & 20 \\
\hline Santa Paula ... & & & & & & & \\
\hline $\begin{array}{l}\text { Area 8-Desert: } \\
\text { Indio. }\end{array}$ & 20 & 3.20 & 73.3 & 88.5 & 57.3 & 125 & 13 \\
\hline
\end{tabular}


MaP 2 - Production areas And harvest seasons for california

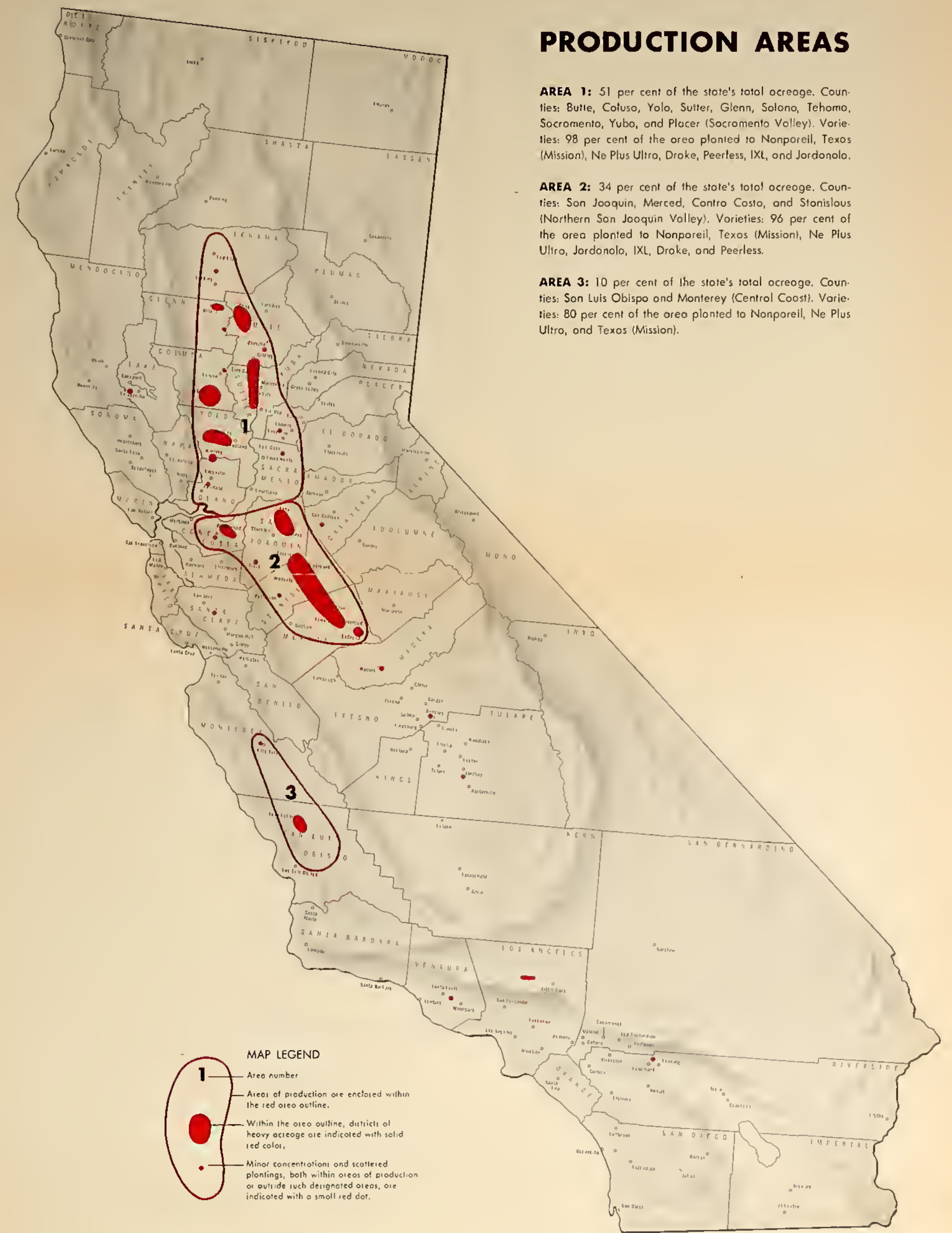

USUAL HARVEST SEASONS

\begin{tabular}{|c|c|c|c|c|c|c|c|c|c|c|c|c|}
\hline AREA AND VARIETY & JAN. & $f \in \mathrm{B}$ & mar. & APR, & MAY & JUNE & juוY & AUG. & sepr. & ocr. & Nov. & $\mathrm{DEC}$ \\
\hline ALL AREAS - & & & & & & & & & & & & \\
\hline AREA $1-$ & & & & & & & & & & & & \\
\hline Nonporeil - & & & & & & & & & & & & \\
\hline Ne Pius Utitro & & & & & & & & & & & & \\
\hline Texas (Missio & & & & & & & 1 & & & & & \\
\hline AREA 2 & & & & & & & & & & & & \\
\hline & & & & & & & & & & & & \\
\hline
\end{tabular}




\begin{tabular}{|c|c|c|c|}
\hline \multicolumn{4}{|c|}{$\begin{array}{l}\text { Table I (Map 2)-Califarnia Almands: Total Aereage (Bearing and } \\
\text { Nanbearing) by Areas and Countles, } 1953\end{array}$} \\
\hline Area and county & $\begin{array}{c}\text { Number of } \\
\text { acreas }\end{array}$ & Area and county & $\begin{array}{c}\text { Namber of } \\
\text { trces }\end{array}$ \\
\hline $\begin{array}{l}\text { Area 1-Sacramento } \\
\text { Valloy: }\end{array}$ & & $\begin{array}{c}\text { Ares 3-Central Cosst: } \\
\text { Monterey. }\end{array}$ & \\
\hline Butto................ & 16,203 & San Luis Obispo.... & 8,376 \\
\hline Coluss. & 9,695 & & \\
\hline Olenn.. & 4,771 & Ares total. & 9,873 \\
\hline Placer...... & 301 & & \\
\hline $\begin{array}{l}\text { Sacramento } \\
\text { Solanno }\end{array}$ & 977 & Additionsl counties : & \\
\hline $\begin{array}{l}\text { Solano ...... } \\
\text { Sutter }\end{array}$ & 2,278 & Los Angeles....... & 2,237 \\
\hline $\begin{array}{l}\text { Sutter... } \\
\text { Telams }\end{array}$ & 6,039 & Fresno.. & $\begin{array}{l}1,375 \\
489\end{array}$ \\
\hline $\begin{array}{l}\text { Teramb. } \\
\text { Yolo.... }\end{array}$ & 1,922 & Makera. & $\begin{array}{l}489 \\
302\end{array}$ \\
\hline \multirow{3}{*}{ Area total. } & $\begin{array}{l}9,402 \\
678\end{array}$ & $\begin{array}{l}\text { Lake... } \\
\text { Tulare. }\end{array}$ & $\begin{array}{l}302 \\
274\end{array}$ \\
\hline & \multirow{2}{*}{52,266} & $\begin{array}{l}\text { Riverside... } \\
\text { Ventura... }\end{array}$ & $\begin{array}{l}269 \\
205\end{array}$ \\
\hline & & Sants Clara. & 186 \\
\hline \multirow{2}{*}{\multicolumn{2}{|c|}{$\begin{array}{l}\text { Area 2-Northern San } \\
\text { Joaquin Valley: }\end{array}$}} & Calaverss. . & 153 \\
\hline & & & 392 \\
\hline Contra Costa........ & 8,306 & Additional counties & \\
\hline Merced..... & $\begin{array}{r}8,706 \\
0006\end{array}$ & total $\ldots \ldots \ldots \ldots$ & 5,882 \\
\hline $\begin{array}{l}\text { San Joaquin. } \\
\text { Stanislaus... }\end{array}$ & $\begin{array}{r}10,260 \\
7,177\end{array}$ & Stato total.... & 102,470 \\
\hline Dismistas.. & & & \\
\hline Ares total.. & 34,449 & & \\
\hline
\end{tabular}

\begin{tabular}{|c|c|c|c|c|}
\hline Varietios & 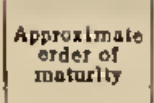 & $\begin{array}{l}\text { Number } \\
\text { of } \\
\text { acren }\end{array}$ & 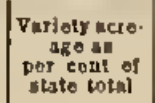 & 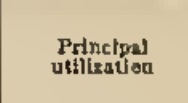 \\
\hline Nonpareil. & 1 & 42,918 & 41.9 & \\
\hline XxL,........ & 2 & $\begin{array}{l}6,304 \\
4065\end{array}$ & 6.1 & All varieties \\
\hline $\begin{array}{l}\text { Jordaluolo.. } \\
\text { Peerless... }\end{array}$ & 4 & $\begin{array}{l}7,000 \\
6,812\end{array}$ & 5.7 & is in shell no \\
\hline Ne Plus UTtrs & 5 & 12,392 & 12.1 & \\
\hline Drake........... & 6 & 8,621 & 8.4 & \\
\hline Texas (Mission). & 7 & 18,262 & 17.8 & \\
\hline Others.......... & $1-7$ & 4,096 & 4.0 & \\
\hline Total. . & & 102,470 & 100.0 & \\
\hline
\end{tabular}

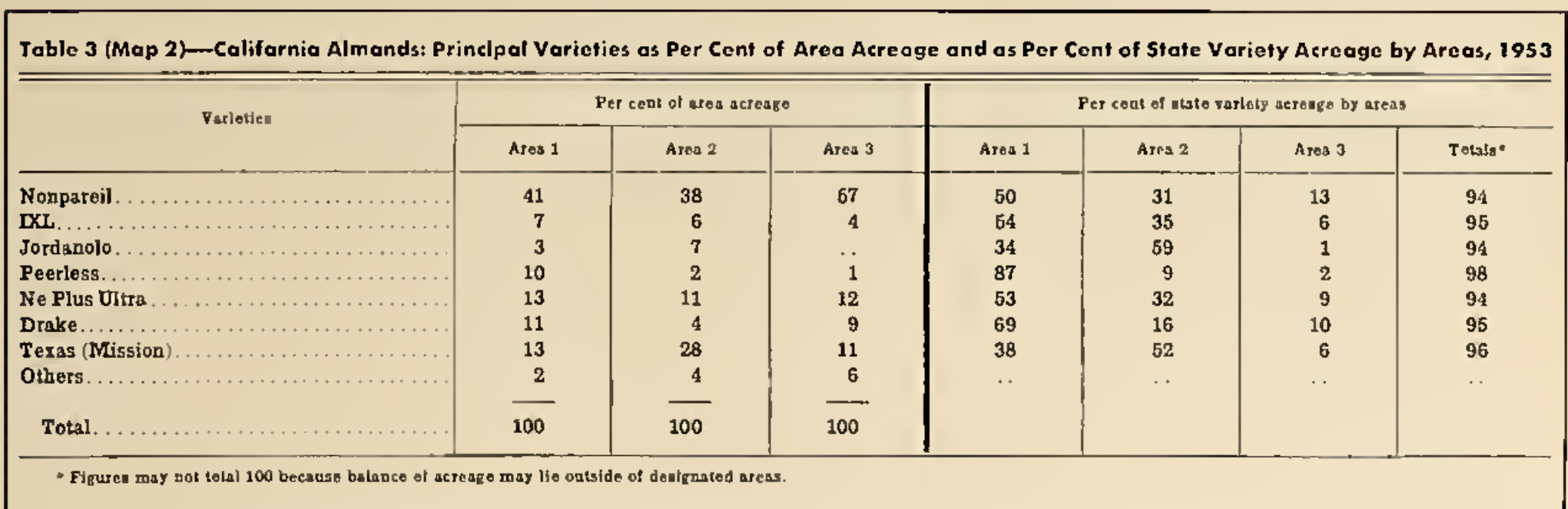

Sources of data: Acreage estimotes in Tables 1 and 2 are based on 1953 total acreage estimates thearing and nonbear. ing) made by the Californio Cro
lished data of the some agency. 
MAP 3 - PROOUCTION AREAS AND haRVESt SEASONS fOR CALIFORNIA A

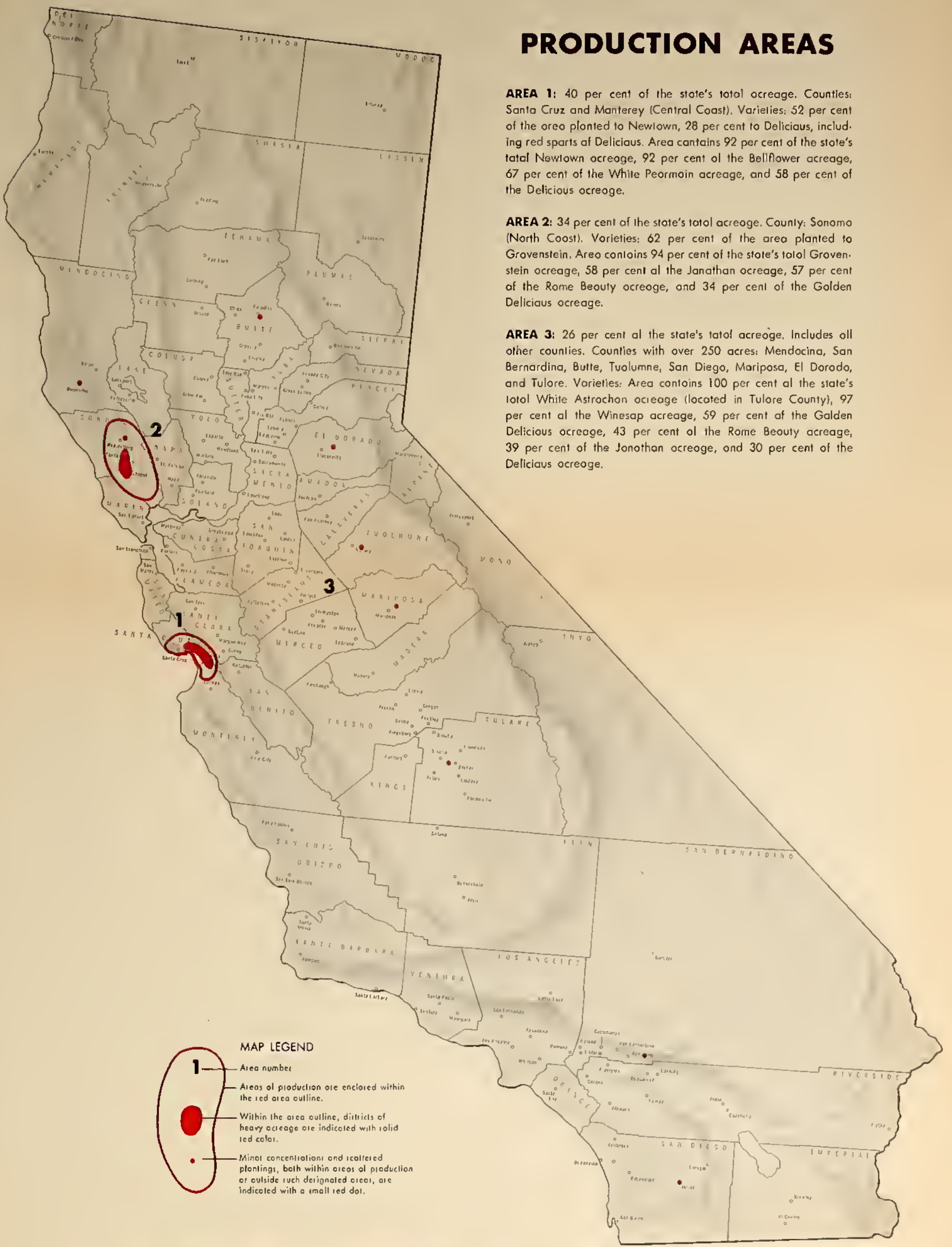

\section{USUAL HARVEST SEASONS}

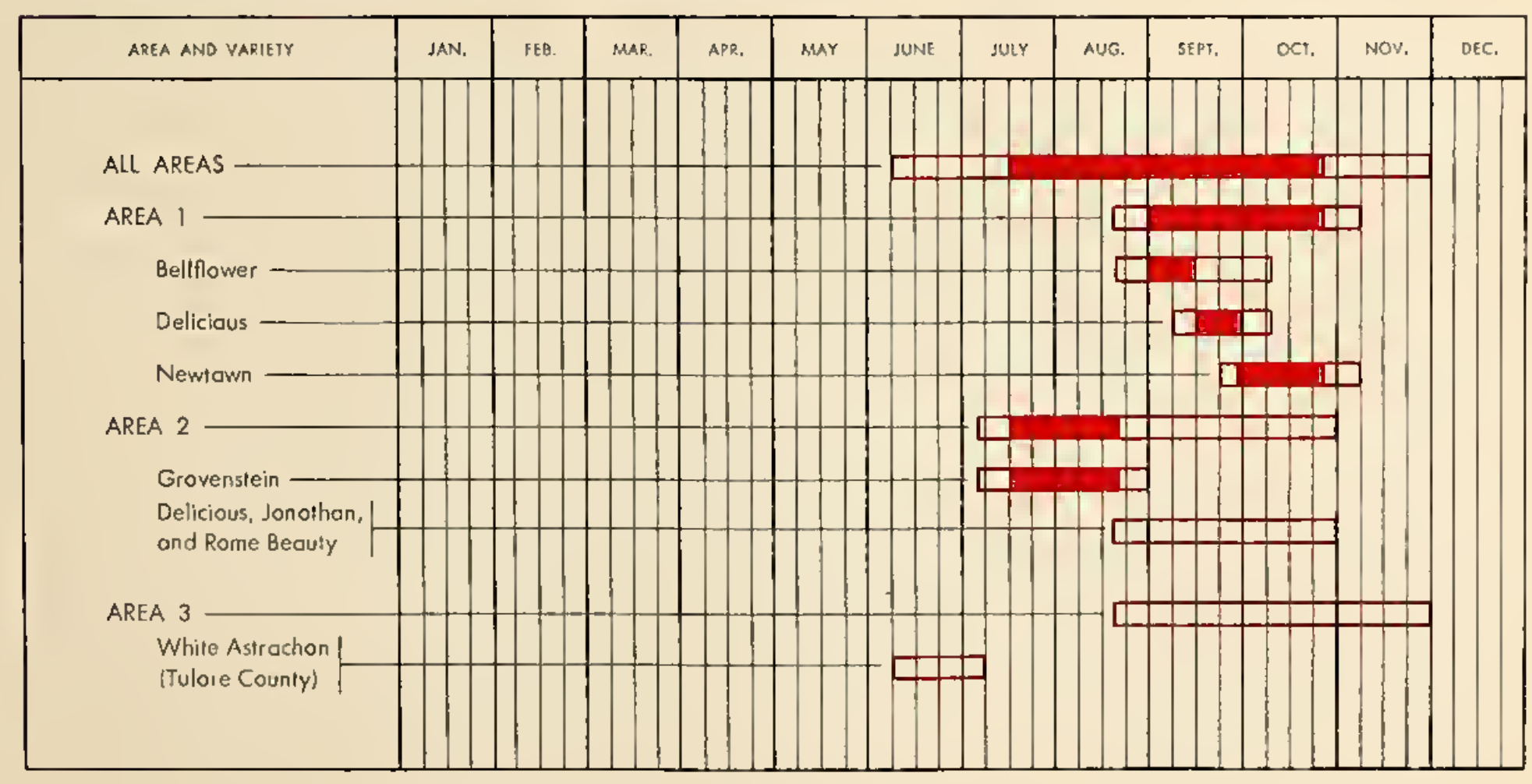




\begin{tabular}{|c|c|c|c|c|c|c|}
\hline \multicolumn{2}{|c|}{$\begin{array}{l}\text { Table I (Map 3)-Californic Apples: Tatal Acreage } \\
\text { (Bearlng and Nanbearing) by Areas and } \\
\text { Counties, 1953 }\end{array}$} & & & & & \\
\hline Ares asd county & $\underset{\substack{\text { Number of } \\
\text { acres }}}{\text { ons }}$ & & & & & \\
\hline $\begin{aligned} \text { Area } 1-\text { Central Coast } \\
\text { Santa Cruz........... } \\
\text { Monterey ............... }\end{aligned}$ & $\begin{array}{r}9,726 \\
768\end{array}$ & & & & & \\
\hline Area total... & 10,494 & & & & & \\
\hline $\begin{array}{l}\text { Ares 2-North Cosst: } \\
\text { Sonons............ }\end{array}$ & 8,910 & \multicolumn{5}{|c|}{$\begin{array}{l}\text { Table } 2 \text { (Map 3)-Califarnia Applest Princlpal Variefles, Maturity, Acreage, } \\
\text { and Utillzatian, } 1953\end{array}$} \\
\hline $\begin{array}{r}\text { Area total...... } \\
\text { Additional counties : }\end{array}$ & 8,910 & Vusletes & 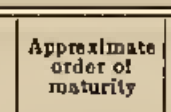 & Number el acros & $\begin{array}{l}\text { Varlety acroage } \\
\text { os per cente of } \\
\text { attate total }\end{array}$ & Prlaclpasl atllizaston \\
\hline $\begin{array}{l}\text { Mendocino........... } \\
\text { San Bemandino }\end{array}$ & 931 & White Astracbsn. & 1 & 222 & 0.8 & \\
\hline Butte........... & 604 & Gravenstein ..... & 2 & 5,807 & 22.2 & Dessert and general purpose \\
\hline Tuolurnne ..... & 575 & Bellfower... & 3 & $\begin{array}{r}980 \\
5097-20\end{array}$ & 3.7 & General purpose \\
\hline San Diego & 472 & Delicious......... & 4 & 5,097 & 19.4 & Dessert \\
\hline Marlposs. & 419 & Gonath Douclous. & 4 & 814 & 3.1 & DesBert and cooking \\
\hline El Dorado. & 308 & Rome Besute..... & 2 & $\begin{array}{l}1,220 \\
1517\end{array}$ & 4.7 & Dessert and general purpose \\
\hline Tulare.... & 263 & Home seauty.... & 4 & $\begin{array}{r}1,647 \\
312\end{array}$ & 5.9 & Baking \\
\hline others ${ }^{*}$. & 2,672 & Newtomn........ & 5 & $\begin{array}{r}312 \\
5.889\end{array}$ & 1.2 & Dessert \\
\hline Additional counties total. & 6,816 & Winesap ........ & 5 & 716 & 2.7 & Dessert and cooking \\
\hline State total .......... & 26,220 & & & & & \\
\hline & & Total.. & & 26,220 & 100.0 & \\
\hline
\end{tabular}

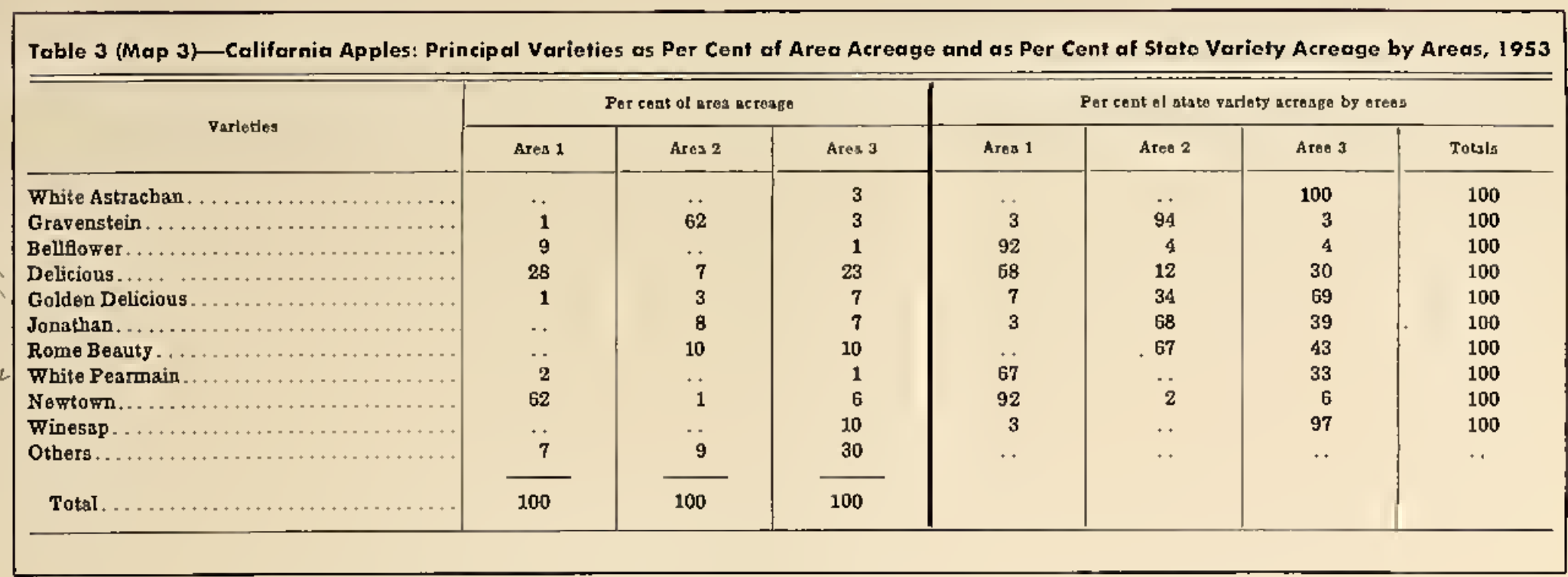

Sources of dalo: Acreoge estimates in Tables 1 and 2 ore based on 1953 Iolal ocreoge eslimales (beoring and nonbeor. ing) made by the Colifiornio Crop
lished data of the same ogency. 
MAP 4 - PRODUCTION AREAS AND HARVEST SEASONS FOR CALIFORNIA A

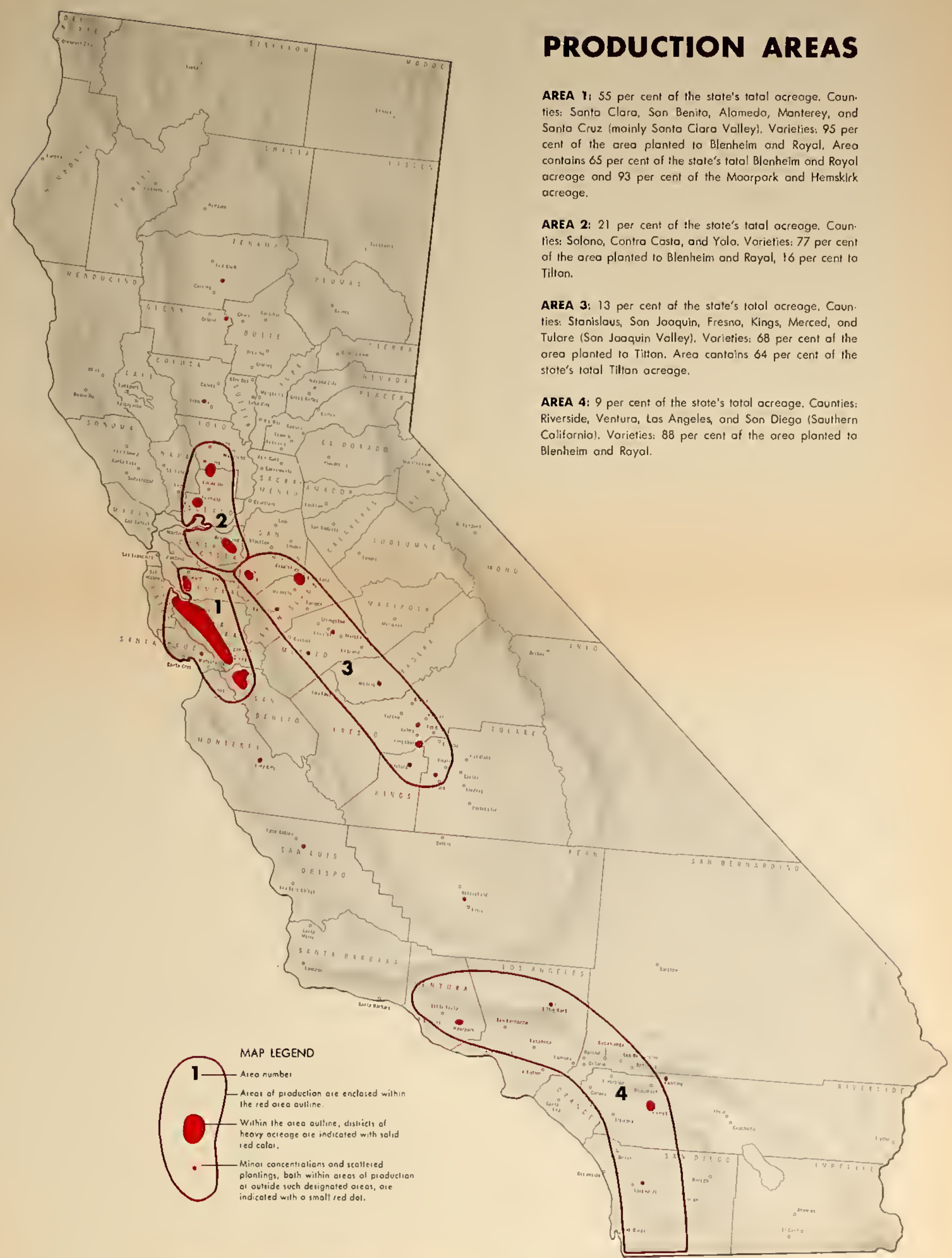

\section{USUAL HARVEST SEASONS}

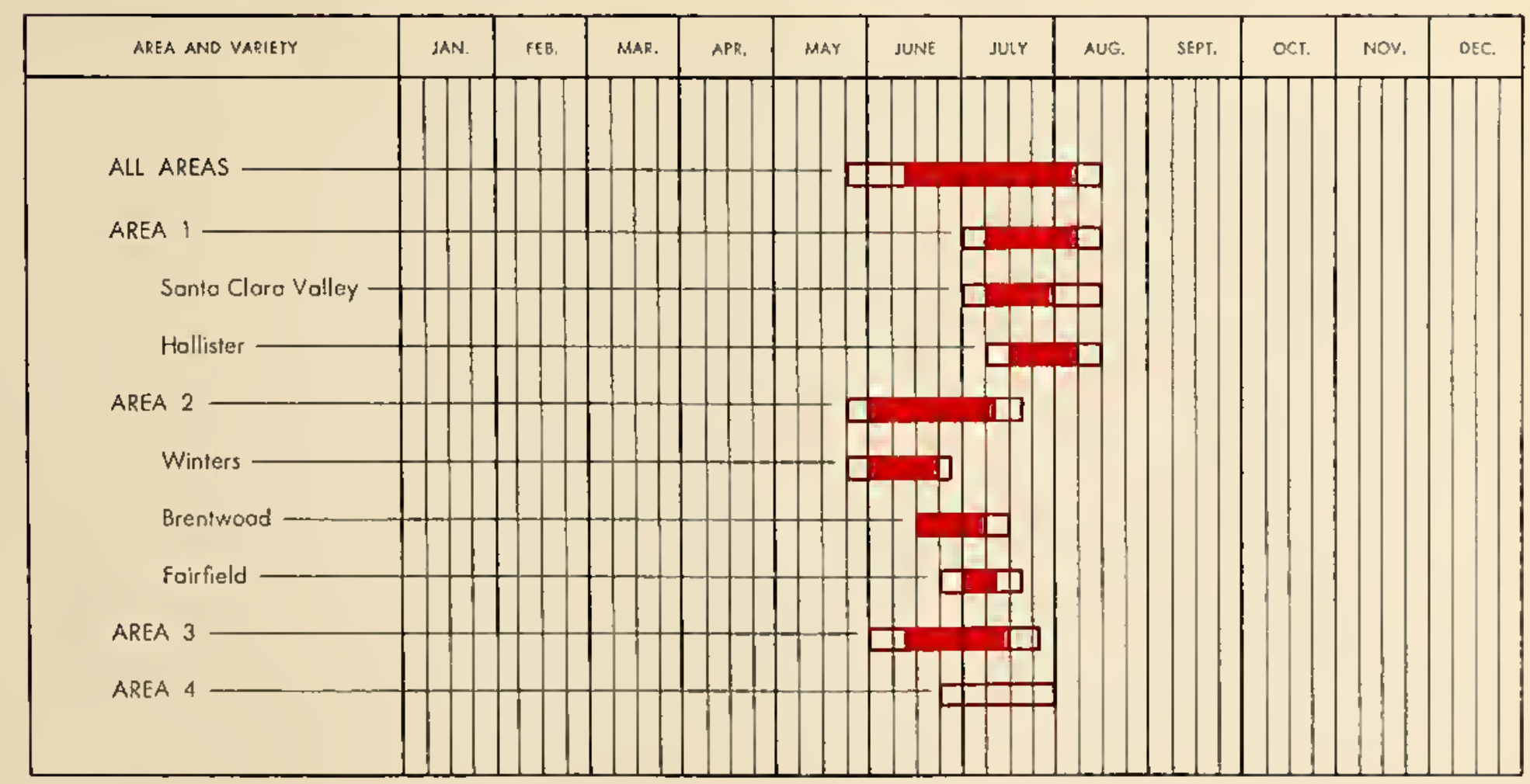

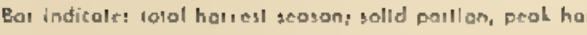




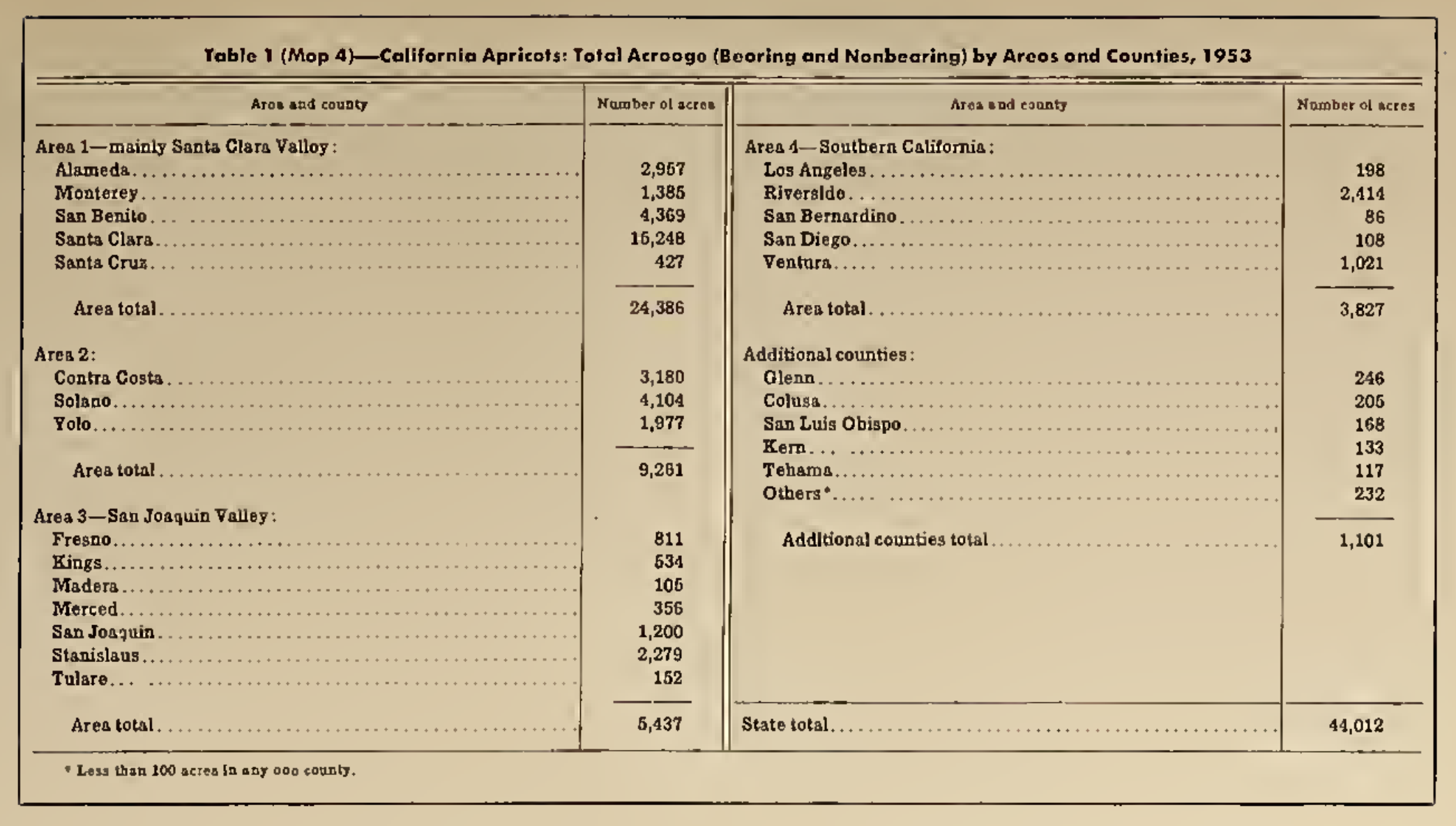

\begin{tabular}{|c|c|c|c|c|}
\hline \multicolumn{5}{|c|}{ Table 2 (Mop 4)—Collfornio Apricots: Principol Vorletles, Maturity, Acreogo, and Utillzotion, 1953} \\
\hline Varlotetics & 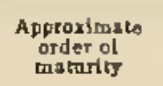 & Number el ecros & 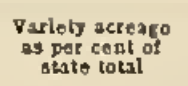 & Prlaclpsl thilibation \\
\hline 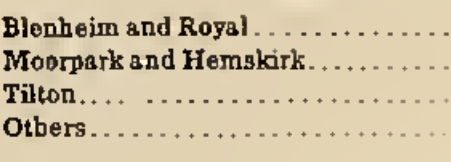 & $\begin{array}{r}1 \\
2 \\
3 \\
1-3\end{array}$ & $\begin{array}{r}36,066 \\
897 \\
5,916 \\
1,133\end{array}$ & $\begin{array}{r}81.9 \\
2.0 \\
13.6 \\
2.6\end{array}$ & $\begin{array}{l}\text { Canned, fresh market, dried, and frozen } \\
\text { Dried, } \\
\text { Canned, dried, fresh market, and frozen } \\
\text { Fresh matket }\end{array}$ \\
\hline Total. . & & 44,012 & 100.0 & \\
\hline
\end{tabular}

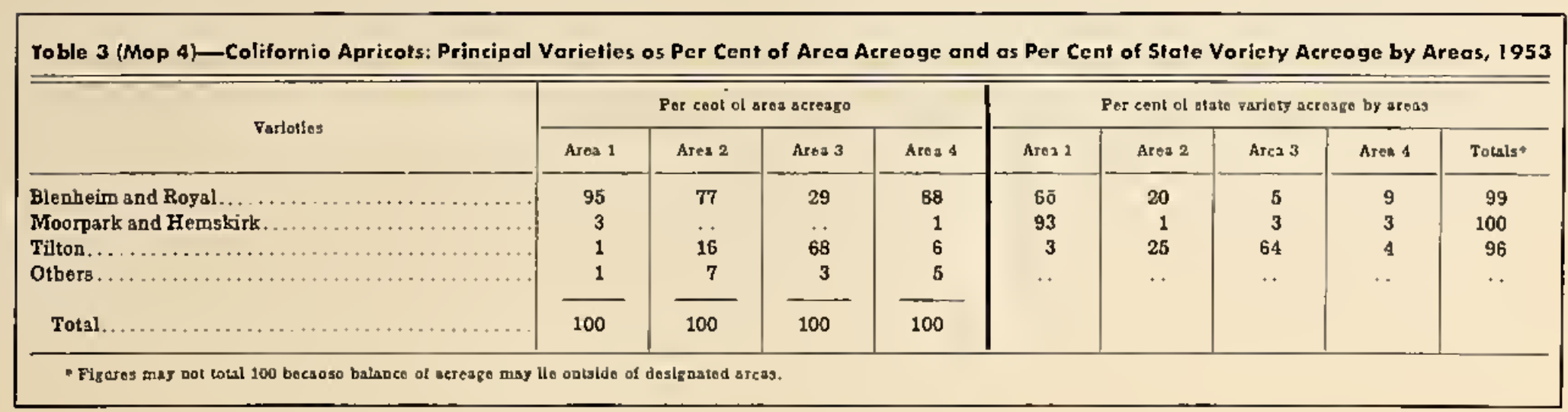

Sources of doto: Acreoge estimates in Tobles 1 and 2 ore bosed on 1953 totol ocreoge eslimoles sbeating ond nonbeoring) mode by the Colfifornio Crop and Livestock Reporting Service. Sociamento. Toble 3 was compiled from unpub-
lished dola of the same agency. 
MAP 5 - PRODUCTION AREAS AND HARVEST SEASONS FOR CALIFORNIA

\section{AVOCADOS}

\section{PRODUCTION AREAS}

AREA 1: 100 per cent of the stote's total acreage. Caunties: San Diega, Los Angeles, Orange, Venturo, Santa Barbara, Riverside, and San Bernordino. Varie.

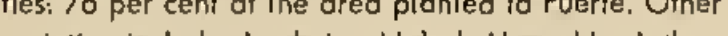

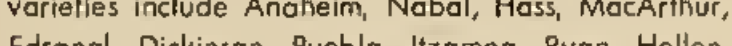
Edranal, Dickinson, Puebla, Itzamna, Ryon, Helien,
Lyon, ond aver 150 others shipped in limiled quanLyon,
tities.
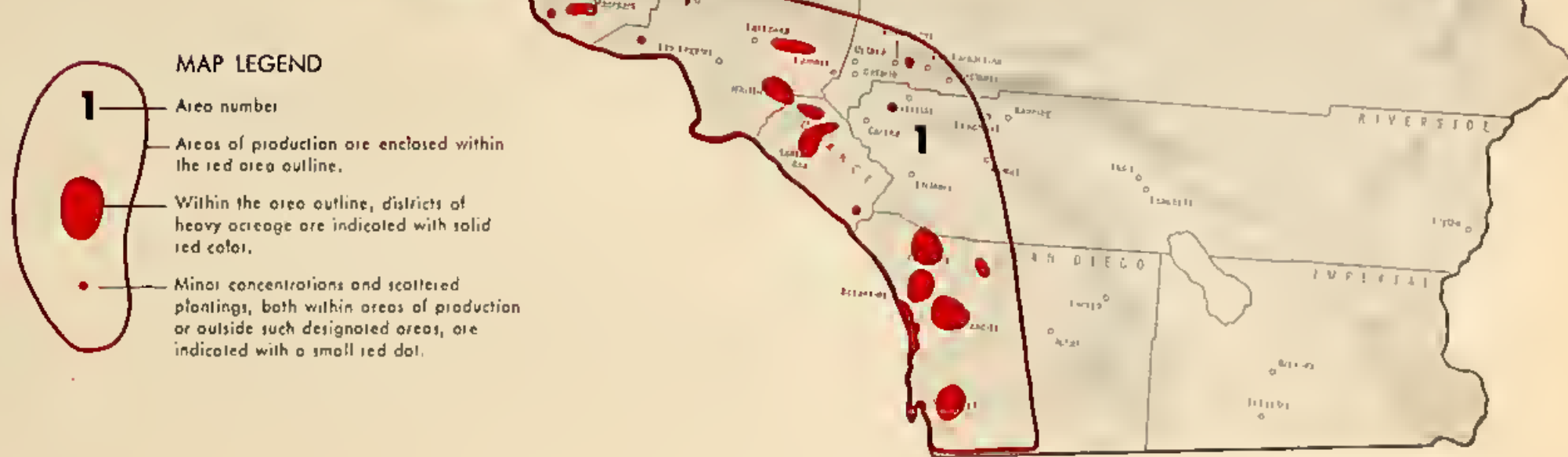

\section{USUAL HARVEST SEASONS}

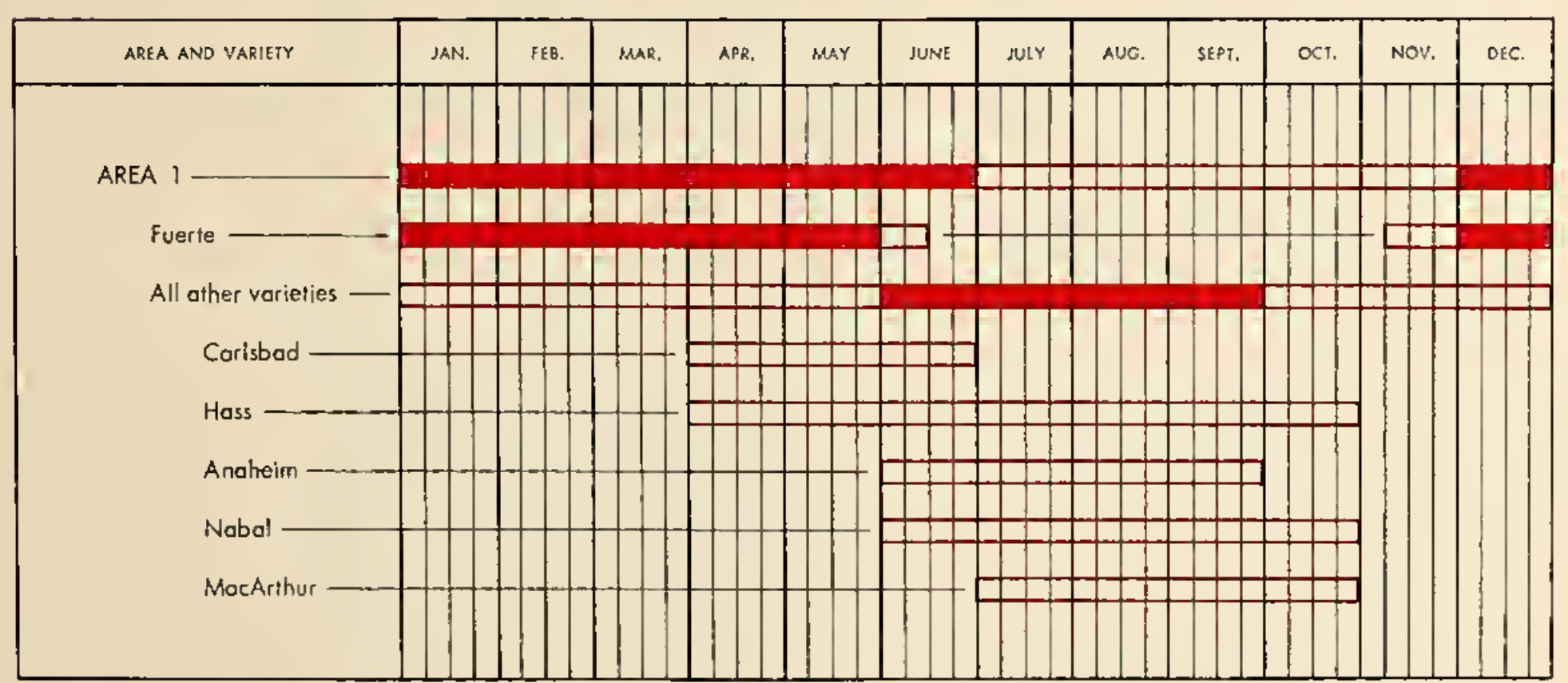




\begin{tabular}{|c|c|}
\hline \multicolumn{2}{|c|}{$\begin{array}{l}\text { Toble I (Map 5)-Califarnia Avatados: Tatal Acreage (Bearing and Nanbearing) } \\
\text { by Areas and Cauntios, } 1953\end{array}$} \\
\hline Area and county & Nambor of seres \\
\hline Area 1-Southem Calitornis: & \\
\hline Los Angeles................. & 2,808 \\
\hline Orange... ... & $\begin{array}{l}2,103 \\
285\end{array}$ \\
\hline Rlversido........ & $\begin{array}{l}280 \\
72\end{array}$ \\
\hline San Bernardino & 13,365 \\
\hline San Diego...... & $\begin{array}{l}13,360 \\
1,245\end{array}$ \\
\hline $\begin{array}{l}\text { Santa Barbara... } \\
\text { Ventura............ }\end{array}$ & $\begin{array}{l}1,245 \\
1,610\end{array}$ \\
\hline Area total........ & 22,088 \\
\hline Additional counties total. . & 18 \\
\hline State total. & 22,106 \\
\hline
\end{tabular}

\begin{tabular}{|c|c|c|c|c|}
\hline Parieties & Seasonst prouds & Number of scres & 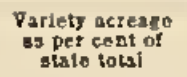 & $\begin{array}{l}\text { Prinicipal } \\
\text { vutilistion }\end{array}$ \\
\hline $\begin{array}{l}\text { Fuerte ..... } \\
\text { Anaheim.... } \\
\text { Nababl...... } \\
\text { Puebla.... } \\
\text { Dickinson. } \\
\text { Others..... } \\
\text { Total.... }\end{array}$ & $\begin{array}{l}\text { Winter and spring } \\
\text { Summer } \\
\text { Summer } \\
\text { Winter and spring } \\
\text { Summer }\end{array}$ & $\begin{array}{r}16,784 \\
390 \\
233 \\
107 \\
60 \\
4,532 \\
22,106\end{array}$ & $\begin{array}{r}75.9 \\
1.8 \\
1.0 \\
0.5 \\
0.3 \\
20.5 \\
100.0\end{array}$ & $\begin{array}{l}\text { All varieties } \\
\text { are marketed } \\
\text { fresh }\end{array}$ \\
\hline
\end{tabular}

Saurces of doto: Acreoge estimates in Tobles 1 and 2 ore bosed on 1953 lolal acreoge estimotes (beoring and nanbeor ing) modo by the Colifornia Crop ond Livestack Reporting Service, Sacromenta. 


\section{PRODUCTION AREAS}

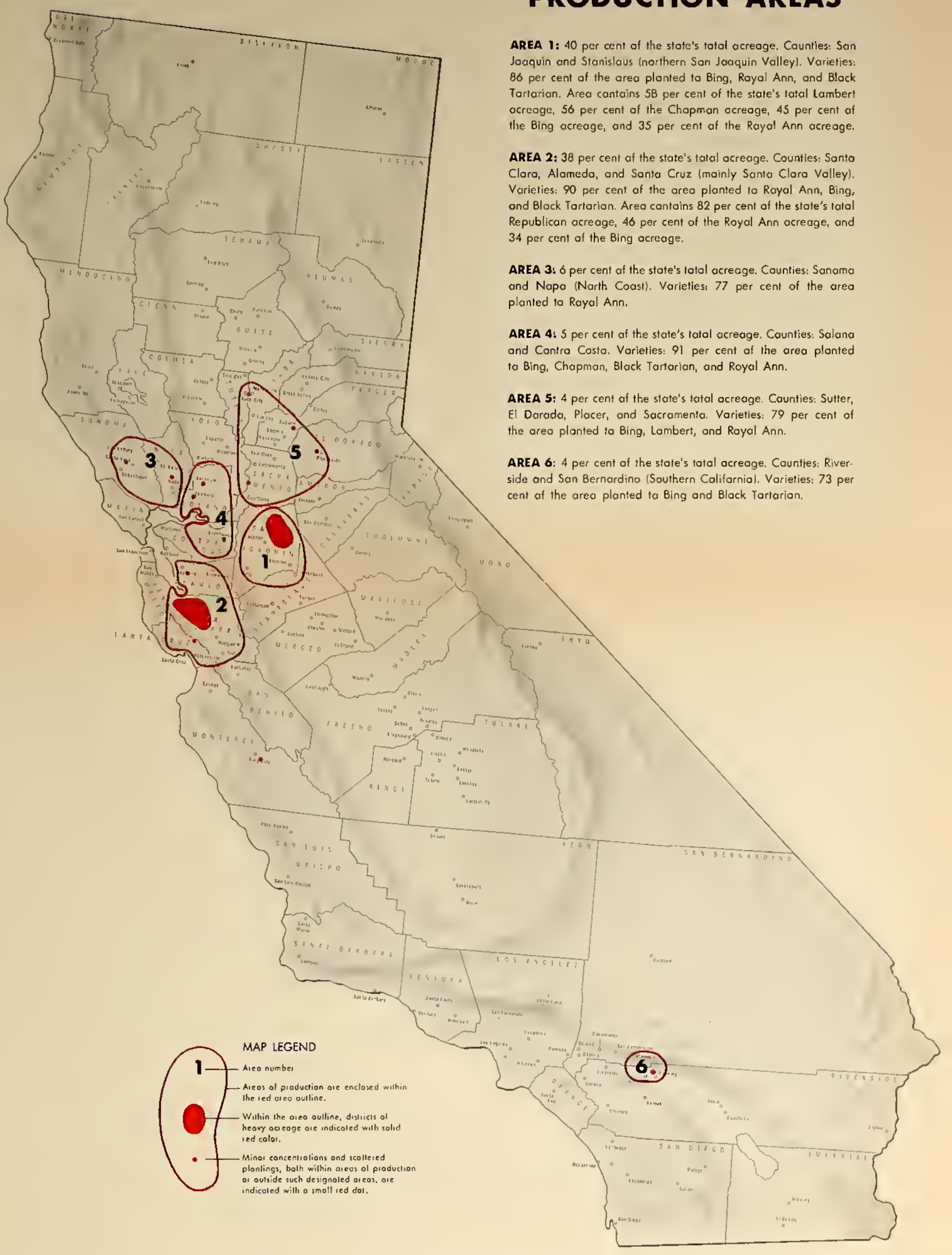

USUAL HARVEST SEASONS

\begin{tabular}{|c|c|c|c|c|c|c|c|c|c|c|c|c|}
\hline AREA AND VARIETY & JAN. & res. & mar. & APR. & mar & JUNE & juLY & AUG. & SEPT. & ост. & Nov. & DEC. \\
\hline ALL AREAS - & & & & & & & & & & & & \\
\hline AREA I - & & & & & & & & & & & & \\
\hline AREA $2-$ & & & & & & & & & & & & \\
\hline AREA $3-$ & & & & & & & & & & & & \\
\hline AREA $4-$ & & & & & & & & & & & & \\
\hline AREA 5 & & & & & & & & & & & & \\
\hline AREA 6 & & & & & & & & & & & & \\
\hline & & & & & & & & & & & & \\
\hline
\end{tabular}




\begin{tabular}{|c|c|c|c|}
\hline Aras and county & Nursiber ot acros & Ares and coanty & Namber at acros \\
\hline Area 1-mainly San Josquin County : & & Area 5: & \\
\hline 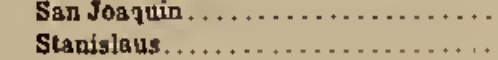 & $\begin{array}{r}4,444 \\
71\end{array}$ & $\begin{array}{l}\text { El Dorado... } \\
\text { Placer...... }\end{array}$ & $\begin{array}{r}123 \\
91\end{array}$ \\
\hline Area total. . . & 4,515 & $\begin{array}{l}\text { Sacramento. } \\
\text { Sutter....... }\end{array}$ & $\begin{array}{r}68 \\
158\end{array}$ \\
\hline Ares 2-majnly Santa Clara Valleg: & & Area total... & 440 \\
\hline Alameda ........................ & 429 & & \\
\hline Santa Clara.. & 3,668 & Area 6: & \\
\hline Santa Cruz.. & 283 & $\begin{array}{l}\text { Riverside......... } \\
\text { San Bernardino. }\end{array}$ & $\begin{array}{r}385 \\
38\end{array}$ \\
\hline Ares total. . & 1,280 & & 4 \\
\hline Area 3: & & & \\
\hline Sonoma.. & 579 & Additional counties: & \\
\hline Napz.... & 93 & $\begin{array}{l}\text { Monterey ... ........... } \\
\text { Otbers }{ }^{\circ} . \ldots \ldots \ldots \ldots \ldots\end{array}$ & $\begin{array}{l}132 \\
228\end{array}$ \\
\hline Area total.... & 672 & Additional counties total. . & 360 \\
\hline $\begin{array}{l}\text { Contra Costa } \\
\text { Solano.......... }\end{array}$ & $\begin{array}{l}265 \\
329\end{array}$ & State total $\ldots \ldots \ldots \ldots \ldots \ldots \ldots \ldots \ldots \ldots \ldots \ldots \ldots \ldots \ldots$ & 11,284 \\
\hline Area total. . & 694 & & \\
\hline
\end{tabular}

\begin{tabular}{|c|c|c|c|c|}
\hline \multicolumn{5}{|c|}{ Table 2 (Map 6)—California Cherries: Principal Varieties, Maturity, Acreage, and Utllizarlon, 1953} \\
\hline Variotics & 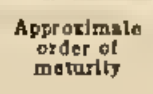 & Nartbor of ecres & 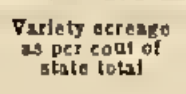 & Princlpal ailiestion \\
\hline $\begin{array}{l}\text { Chapman......... } \\
\text { Blsck Tartarian... } \\
\text { Bing .......... } \\
\text { Royal Ann....... } \\
\text { Republican ....... } \\
\text { Lambert......... } \\
\text { Otbers......... }\end{array}$ & $\begin{array}{r}1 \\
2 \\
3 \\
4 \\
5 \\
6 \\
6 \\
1-7\end{array}$ & $\begin{array}{r}300 \\
1,609 \\
4,330 \\
3,688 \\
232 \\
496 \\
629\end{array}$ & $\begin{array}{r}2.6 \\
14.2 \\
38.4 \\
32.7 \\
2.1 \\
4.4 \\
5.6\end{array}$ & $\begin{array}{l}\text { Fresh market } \\
\text { Fresb market } \\
\text { Fresh market, canned, and brined } \\
\text { Brined, canned, and fresb market } \\
\text { Brined } \\
\text { Fresh market } \\
\text { Fresh market }\end{array}$ \\
\hline Total... & & 11,284 & 100.0 & \\
\hline
\end{tabular}

\begin{tabular}{|c|c|c|c|c|c|c|c|c|c|c|c|c|c|}
\hline \multirow{2}{*}{ Vartetsics } & \multicolumn{6}{|c|}{ Per cout of areas acteasto } & \multicolumn{7}{|c|}{ Por cont of state parloyly acreago by erees } \\
\hline & Aros 1 & Areo 2 & Aroa 3 & Area 4 & Asea is & Areo 6 & Area 1 & Ares 2 & Aroo 3 & Aroo 4 & Aroos 5 & Areo 6 & Totalls* \\
\hline Chapmas ...... & 4 & & & 19 & 4 & & 56 & 1 & & 37 & 6 & & 100 \\
\hline Black Tartarian. & 14 & 16 & 4 & 13 & 7 & 32 & 39 & 41 & 2 & 5 & 2 & 8 & 97 \\
\hline Bing........... & 43 & 35 & 7 & 49 & 47 & 41 & 45 & 34 & 1 & 7 & 5 & 4 & 96 \\
\hline Royal Ann. & 29 & 39 & 77 & 10 & 15 & 7 & 35 & 46 & 14 & 2 & 2 & 1 & 100 \\
\hline $\begin{array}{l}\text { Republican. } \\
\text { Rent }\end{array}$ & 1 & 4 & .. & 20 & 10 & 1 & 12 & 82 & 19 &. & a & 1 & 96 \\
\hline Lambert.... & 6 & 1 & . & & 17 & 11 & 58 & 8 & .. & .. & 15 & 9 & 90 \\
\hline Others... & 3 & 5 & 12 & 9 & 10 & B & .. & .. & .. & .. & & & \\
\hline Total... & 100 & 100 & 100 & 100 & 100 & 100. & & & & & & & \\
\hline
\end{tabular}

Saurces of dota: Acreoga estimoles in Tobles 1 ond 2 ore bo sed on 1953 latol ocreoge eslimoles (beoring and nanbeor. ing) made by the Colifornio Crop and Livestack Reporling Service, Socromento. Toblo 3 was compiled from unpub-
lished dalo of the some ogency. 
MAP 7 - PRODUCTION AREAS AND HARVEST SEASONS FOR CALIFORNIA

\section{DATES}

\section{PRODUCTION}

AREAS

AREA 1: 99 per cent of the stole's lotal ocreoge. Counties: Riverside (eostern), Imperial and Son Diego (eosiern) (moinly Coochello Volley). Vorieties: 83 per cent of the oreo plonted 10 Deglel Noor, 4 per cent 10 Zohidi, and 3 per cent to Khadrowy.
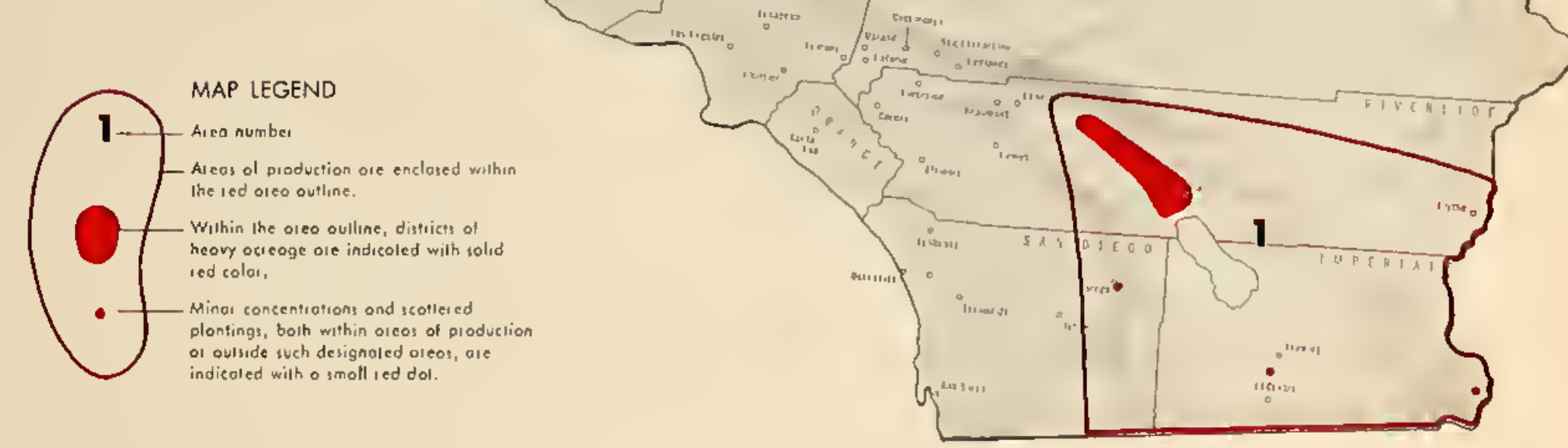

USUAL HARVEST SEASONS

\begin{tabular}{|c|c|c|c|c|c|c|c|c|c|c|c|c|}
\hline AREA AND VARIEZY & JAN. & fEE. & MAR. & APR & MAY & JUNE & jutr & AUG & SEPT & oct. & Nov & OEC. \\
\hline AREA $1=$ & & & & & & & & & & & & \\
\hline Khodrowy - & & & & & & & & & 些 & +1 & & \\
\hline Zohidi - & & & & & & & & & & & & \\
\hline Deoler Noar & & & & & & & & & & & & \\
\hline & & & & & & & & & & & & \\
\hline
\end{tabular}




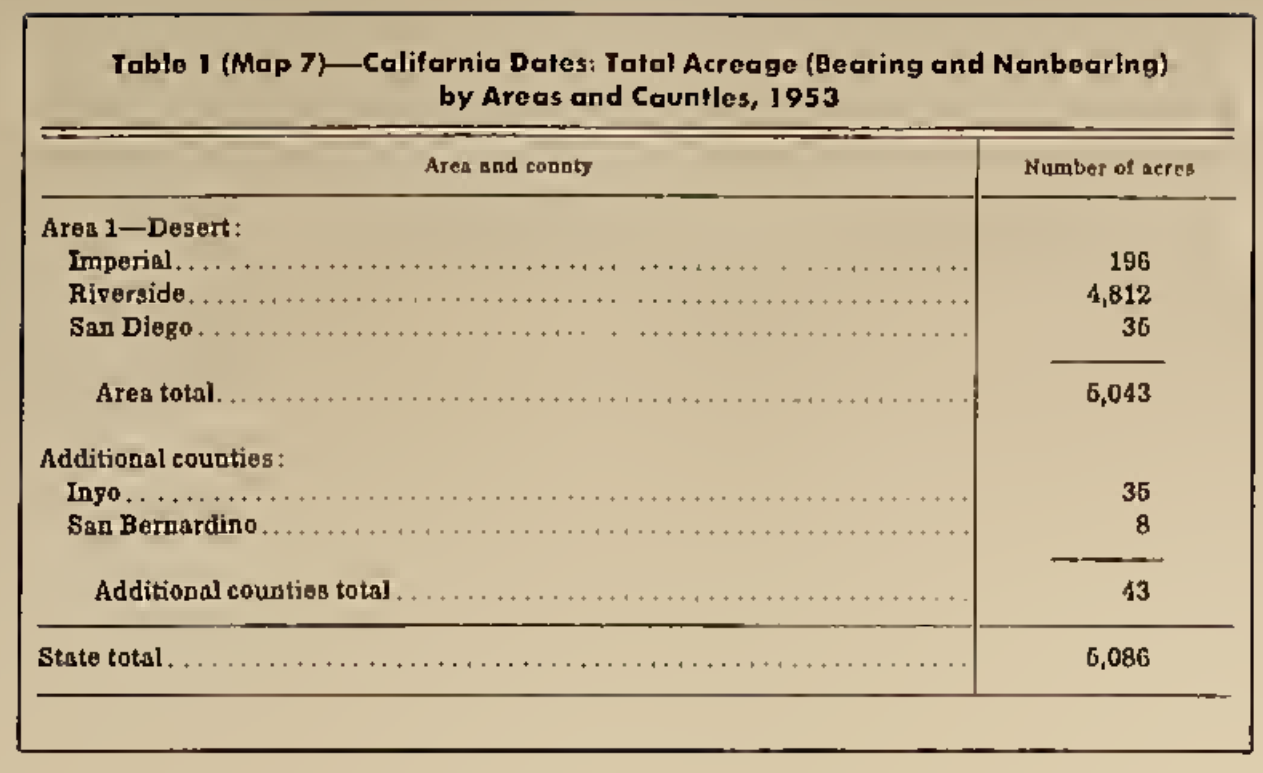

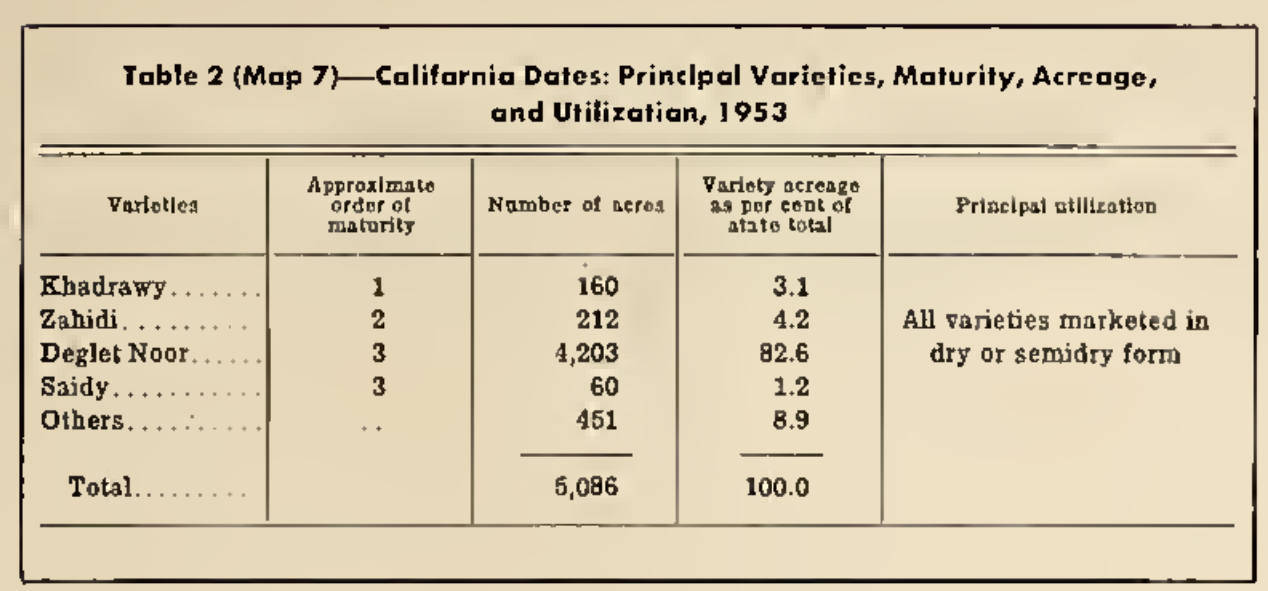

Sources of dola: Acreage estimates in Tobles 1 and 2 ore bosed on 19531010 ocreage eslimoles (bearing ond nonbear 
MAP 8 - PRODUCTION AREAS AND HARVEST SEASONS FOR CALIFORNIA

\section{PRODUCTION AREAS}

AREA 1: 64 per cent of the stote's totol acreage. Coun. lies: Fresna, Madero, ond Tulare. Vorieties: 51 per cent of the oreo plonled to Colimyrno, 34 per cent to White Adriatic. Areo contoins 84 per cent of the state's totol Collmyrno acreoge and 75 per cent of the White Adriotic acreoge.

AREA 2; 29 per cent of tho stote's fotol ocreoge. County: Merced. Vorieties: 43 per cent of the areo planted to Kodolo. Areo contoins 63 per cent of the stole's totol Kodoto ocreoge,

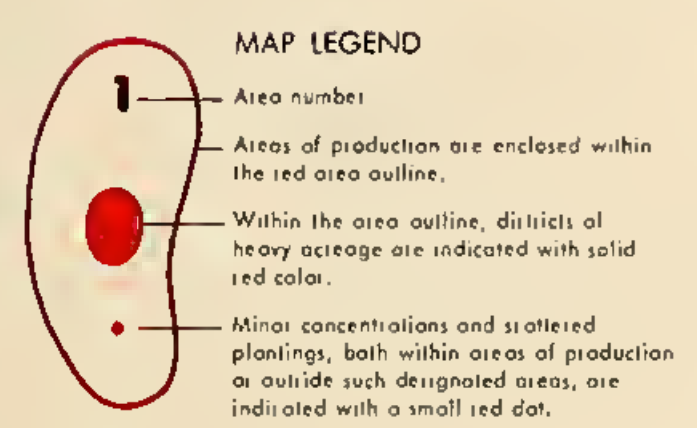




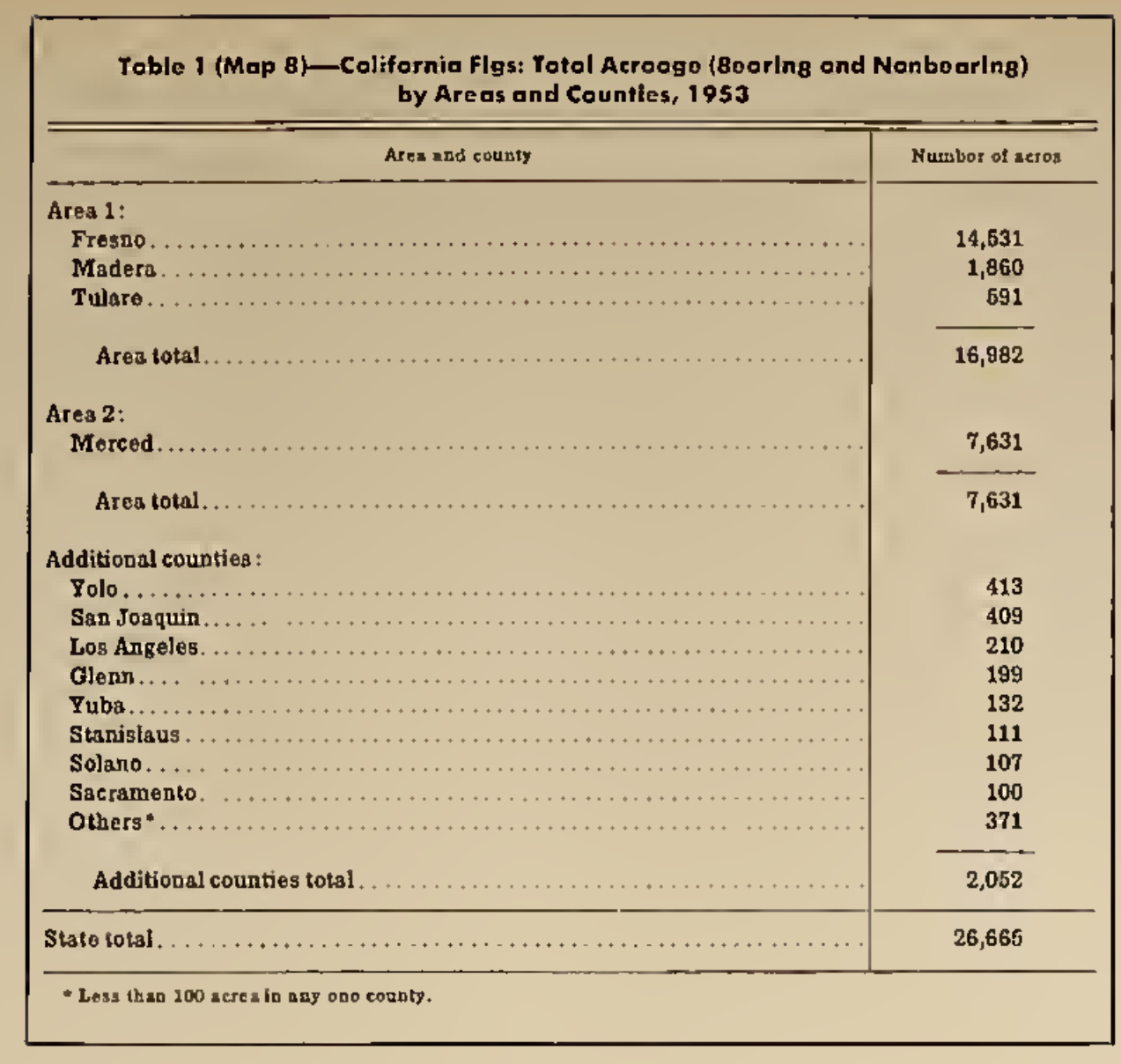

\begin{tabular}{|c|r|r|r|r|}
\hline \multicolumn{5}{|c|}{ Table 2 (Mop 8)-Colifornio Figs: Principal Varieties, Maturity, Acreage, } \\
ond Utilization, 1953
\end{tabular}

Sources of doto: Acreage estimotes in Toblës 1 and 2 ore based on 1953 torol ocreoge estimotes theoring and nonbeor. ing) mode by the Calilornia Crop and Livestock Reparting Service, Socromento. 


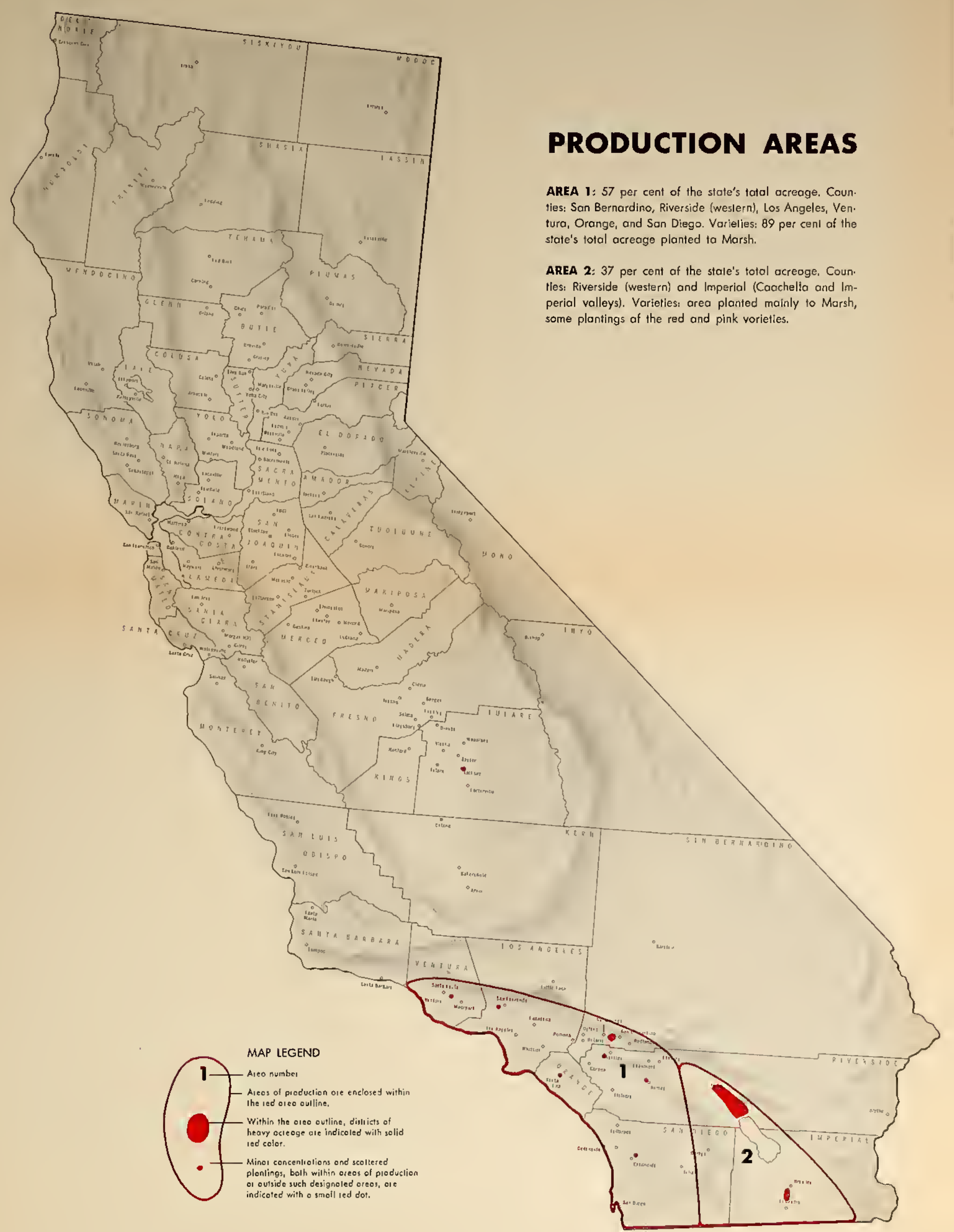

USUAL HARVEST SEASONS

\begin{tabular}{|c|c|c|c|c|c|c|c|c|c|c|c|c|}
\hline AREA AND VARIETY & JAN. & res. & MAR. & APR. & mar & JUNE & surr & AUG. & sept. & ост. & Nov. & DEC. \\
\hline All AREAS & & & & & & & & & & & & \\
\hline AREA $1-$ & & & & & & & D & D & ل & & & \\
\hline AREA 2 & & & & & & & & & & & & \\
\hline & & & & & & & & & & & & \\
\hline
\end{tabular}

GRAPEFRUIT- - Map 9 of Manuol 20

"The Where and When of Colifornia Fruit and Nut Crops" by Robert C. Rock and A. D. Rizzi. 


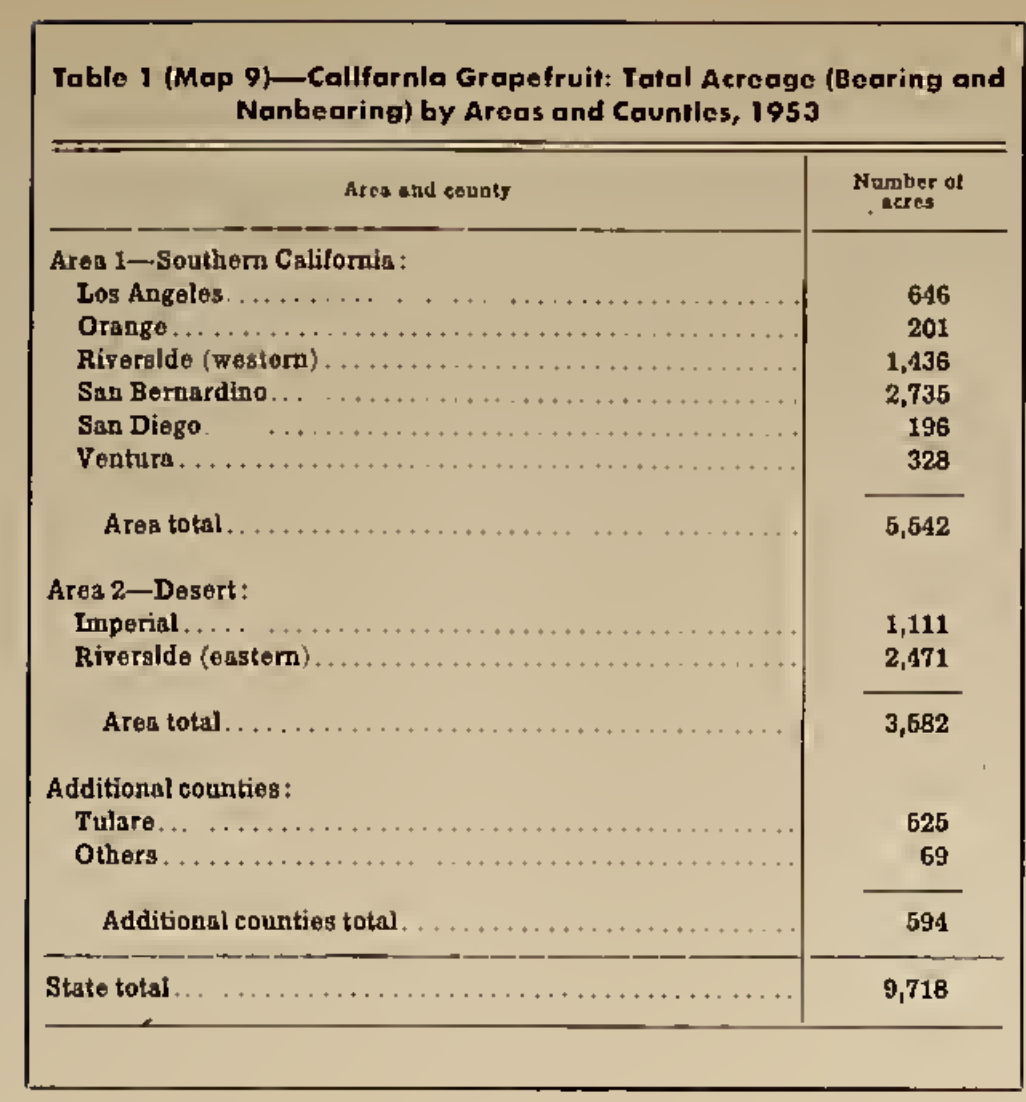

\begin{tabular}{|c|c|c|c|}
\hline \multicolumn{5}{|c|}{ Table 2 (Map 9) Cackilifarnla Grapefruit: Principal Varieties, } \\
Acreagc, and Utllizallan, 1953
\end{tabular}

Sources of dato: Acreage estimates in Tobles 1 ond 2 ore bosed on 1953 total acreage estimoles (bearing and nonbeor (ing' mode by the Colifornia Crop ond Livestock Reporting Service. Sacromento. 


\section{GRAPES}

\section{PRODUCTION AREAS}

AREAS 1-6: 80 per cent of the stote's totol ocreoge ISon Jooquin Vol. AREA 2: 12 per cent of the stote's totol ocreage. County: Son Jooquin.

AREA 1: I per cent of the stote's lotof ocreoge. Counties: Sacramento.

AREA 2: 12 per cent of the slote's totol ocreoge. Caunly: Son Jooquin.
Vorieties: 41 per cent of the oreo plonted to Takay, 56 per cent to wine varieties. Area cantoins 92 per cent of the stote's lotal Tokoy ocreage. AREA 3: 7 per cent of the stote's totat ocreoge. Counties: Stanislous AREA 3: 7 per cent of the stote's totat ocreoge. Counties: Stanislous
and Merced. Varieties: 49 per cent of the orea planted to Thomosan Seedless and 42 per cent to wine vorieties.

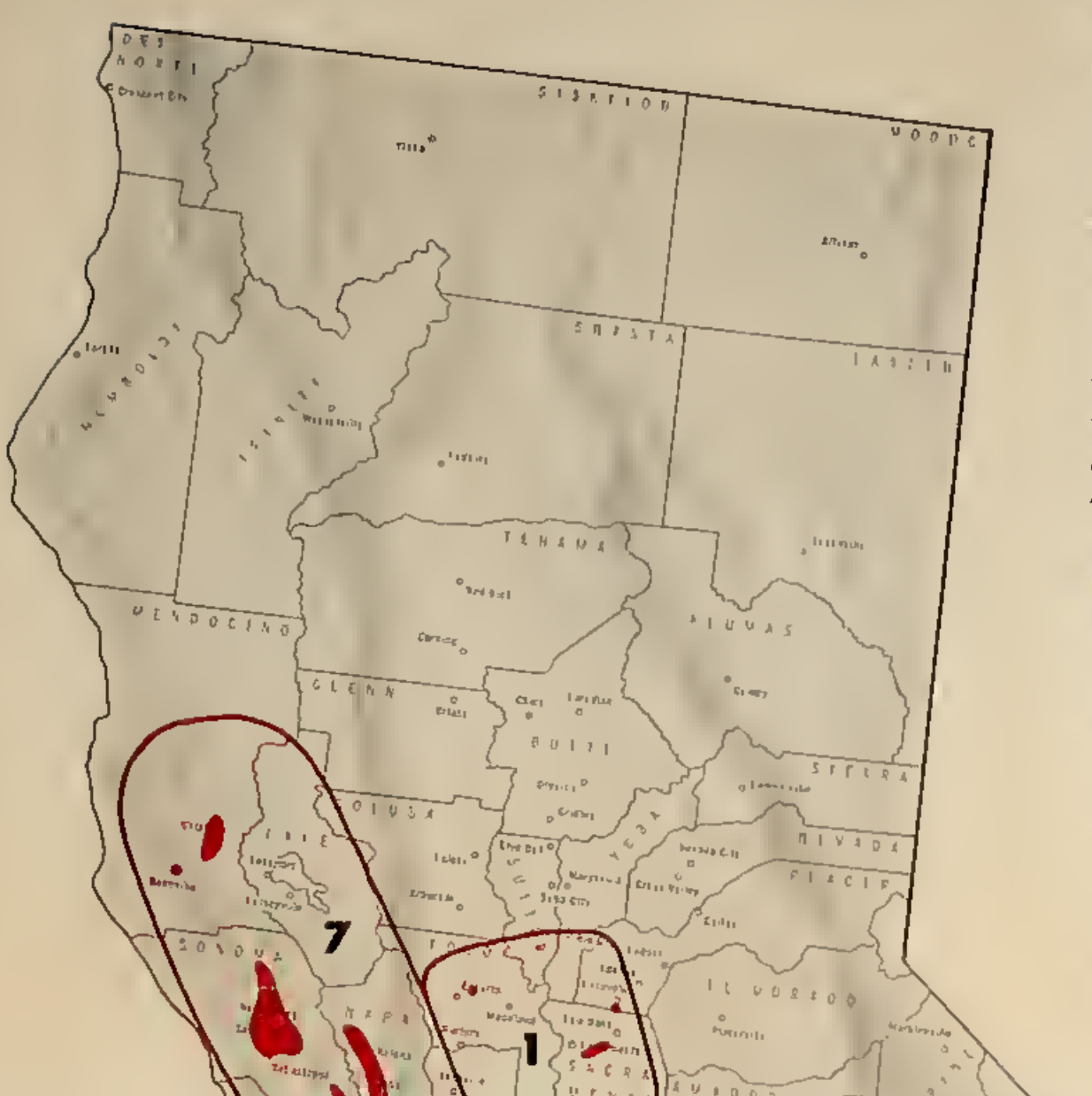

AREA 4: 39 per cent of the state's total ocreoge. Counties: Fresna and A per ceet of the Muscol orreoge, 65 per cent of the Thompson 5 sed 67 acreage, 62 per cent of the Sultono ocreage 55 per cent of the While Mologo ocreage, and 25 per cent of the Cordinol ocreage.

AREA S: 15 per cent of the state's totof acreage. Caunties: Tulare and Kings. Vorieties: 40 per cent of the oreo plonted to Thompson Seedless ond 28 per cent to Emperar. Tulore Caunty contains 65 per cent of the stote's total Emperar acreage and 36 per cent of the Ribier ocreage.

AREA 6: 6 per cent of the stote's totol ocreoge. County: Kern. Vorieties 44 per cent of the oree planted to Thompson Seedless. Area contatns 44 per cent of the stote's fotol Red Mologa ocreage ond 33 per cent of the Ribier ocreage.

AREAS 7\& 8: 10 per cent of the stote's totol ocreoge (Norit Caost ond Central Coosti.

AREA 7:7 per cent af the state's total ocreoge. Counties. Sonomo, Nopo, Mendacina, Solona, Marin, ond Lake. Vorieties: oreo plonted exclusively to

AREA 8: 3 per cent of the stote's to:ol acreoge. Countiesi Santo Claro. Cantro Casto, Alamedo, San Benito, San Luis Obispo, Sonto Cruz, ond A Varieties: oreo planted exclusively to wine vorieties.

AREA 9: 8 per cent of the stote's torol ocreage. Caunties: Son Bernordino, Riverside (western), Son Diega (western) ond Los Angefes. Vorieties: 82 per cent af area planted to wine varieties.

AREA 10: 2 per cent of the stote's totol acreoge. Counties: Riverside (eastern), Son Diego (eostern), and Imperiol 86 per cent ol oreo planted to Thomp. son Seedless.

Areo contoins 27 per cent of the stote's lotol Cordinol acreage ond plonting

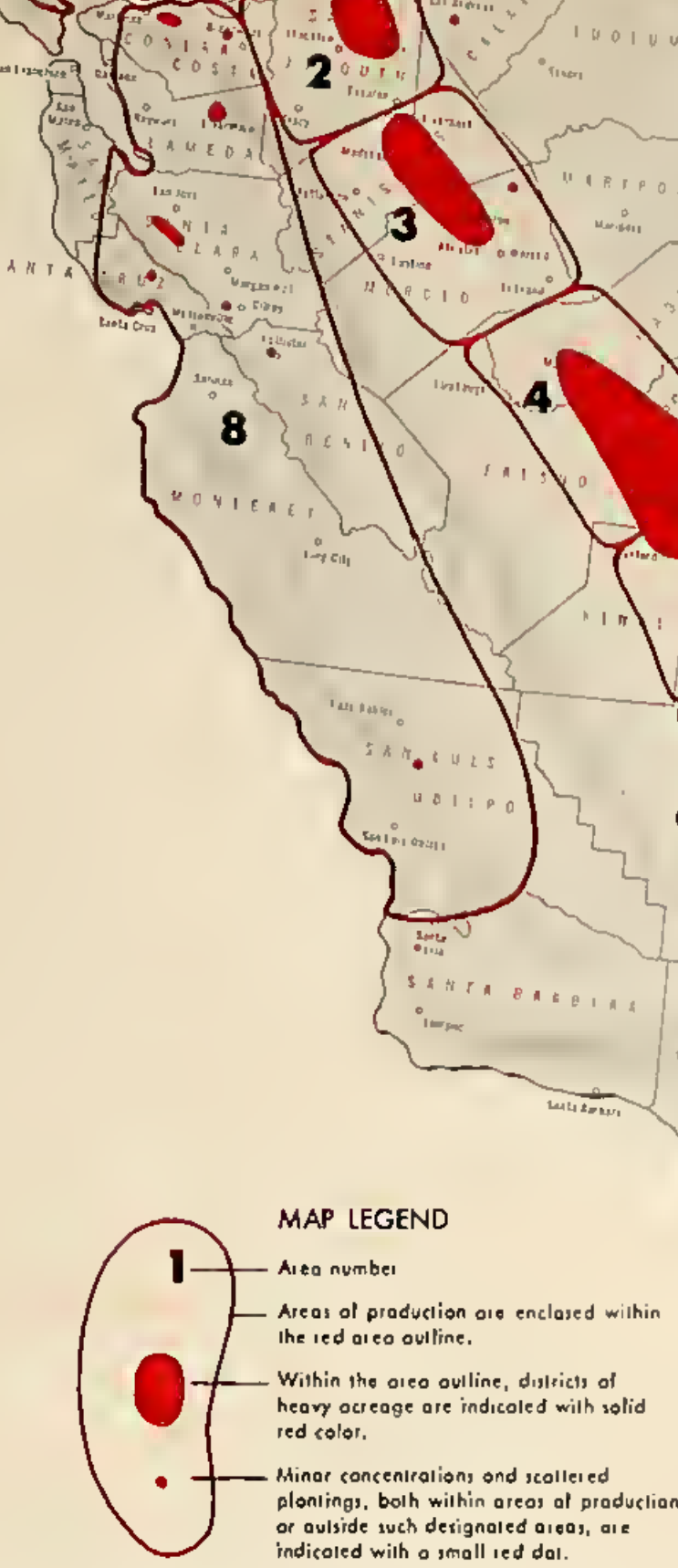

USUAL HARVEST SEASONS

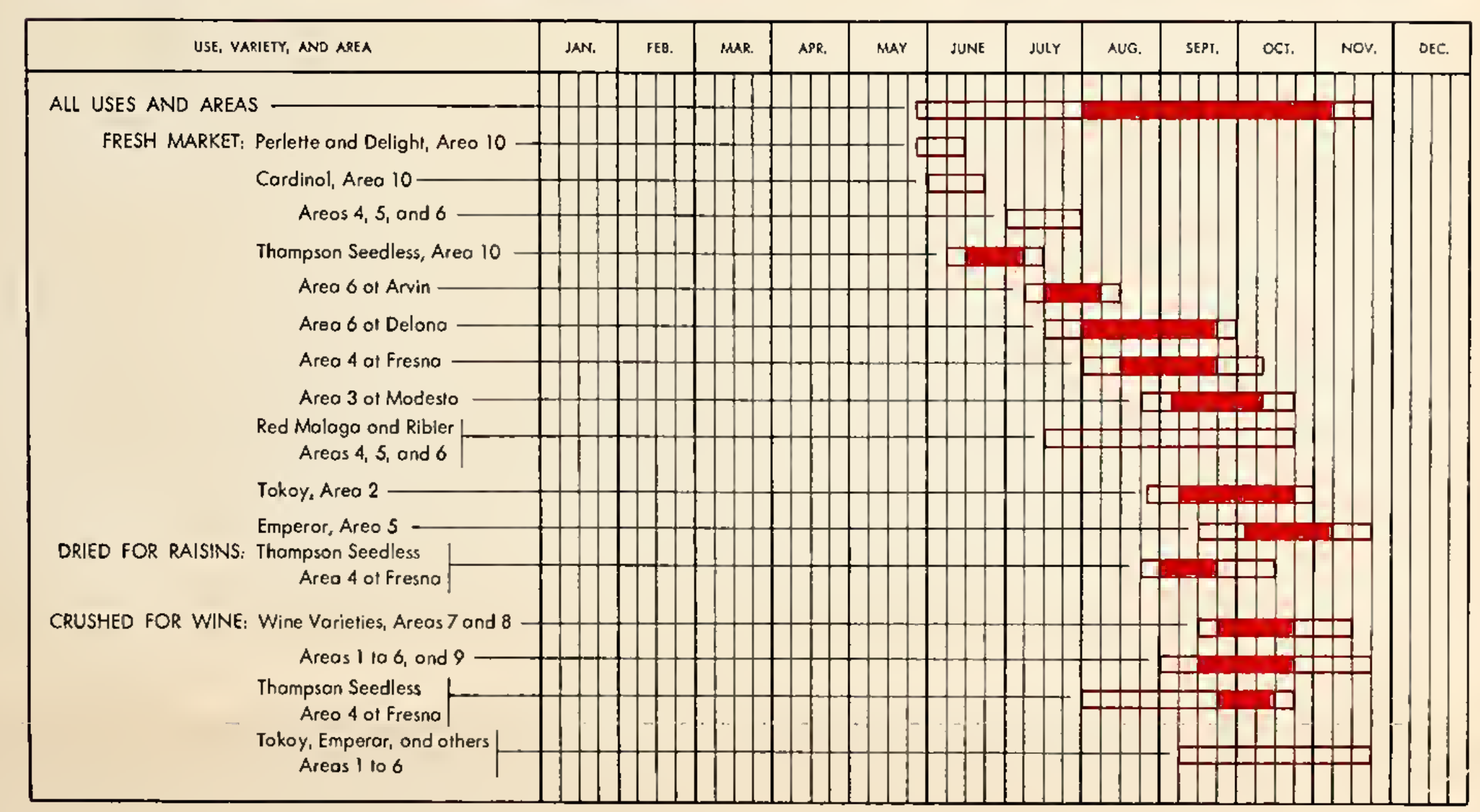




\begin{tabular}{|c|c|c|c|}
\hline Aresend county & Number of acroes & Ares and coninty & Number ol actes \\
\hline Area 1: & & Area 7-Nortb Coast: & \\
\hline Placer ..... & & Lake.............. & 169 \\
\hline Sacramento & 2,037 & Marin........... & \\
\hline Yolo........ & & $\begin{array}{l}\text { Mendacino..... } \\
\text { Napa........... }\end{array}$ & $\begin{array}{r}6,722 \\
10,626\end{array}$ \\
\hline Ares total.. & 3,469 & $\begin{array}{l}\text { Solano.............. } \\
\text { Sonom. }\end{array}$ & 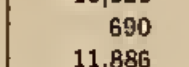 \\
\hline Area 2: & & Sonoma.... & \\
\hline San Josquin... & 56,284 & Area total... & 30,240 \\
\hline Area total... & 56,284 & $\begin{array}{l}\text { Area } 8 \text {-Central Coast: } \\
\text { Alameda }\end{array}$ & \\
\hline Area 3: & & Contra Costa............. & 3,513 \\
\hline $\begin{array}{l}\text { Mereced.......... } \\
\text { Stanisluus...... }\end{array}$ & $\begin{array}{l}16,274 \\
16,273\end{array}$ & $\begin{array}{l}\text { Monterer......... } \\
\text { San Benito..... }\end{array}$ & $\begin{array}{r}119 \\
1,186\end{array}$ \\
\hline \multirow[t]{3}{*}{ Area total. . } & 31,547 & $\begin{array}{l}\text { San Luis Ohispo... } \\
\text { Santa clars ..... } \\
\text { Santa Cruz ........... }\end{array}$ & $\begin{array}{r}707 \\
6,150 \\
572\end{array}$ \\
\hline & & Area total.... & 14,274 \\
\hline & & Areas 7 and 8 total - North and Central Coast ...... & .44,514 \\
\hline Ares 4: & & Area 9-Southem California: & \\
\hline $\begin{array}{l}\text { Fresno ....... } \\
\text { Madera..... }\end{array}$ & $\begin{aligned} 162,787 \\
1809\end{aligned}$ & $\begin{array}{l}\text { Los Angeles............... } \\
\text { Riverside (western) }\end{array}$ & $\begin{array}{l}1,307 \\
3,86\end{array}$ \\
\hline Aros total. . & 180,796 & $\begin{array}{l}\text { San Bernardino............ } \\
\text { San Diego (western).... }\end{array}$ & 29,653 \\
\hline Area 6: & & Area total.... & 36,892 \\
\hline $\begin{array}{l}\text { Ringa ..... } \\
\text { Tulare .... }\end{array}$ & $\begin{array}{r}5,230 \\
66,002\end{array}$ & & \\
\hline & & Imperial........... & 1,220 \\
\hline Areas total... & 71,232 & $\begin{array}{l}\text { Riverside (eastem) } \\
\text { San Diego (eastern). }\end{array}$ & $\begin{array}{l}8,884 \\
1,610\end{array}$ \\
\hline $\begin{array}{l}\text { Area 6: } \\
\text { Bern. }\end{array}$ & 26,647 & Ares total......... & 11,714 \\
\hline Area total. . & 26,647 & Additional coundies total ... & 1,946 \\
\hline Areas 1-6 total (San Joaquin Valley) & 368,966 & State total $\ldots \ldots \ldots \ldots \ldots \ldots \ldots$ & 464,031 \\
\hline
\end{tabular}

\begin{tabular}{|c|c|c|c|c|}
\hline \multicolumn{5}{|c|}{ Toble 2 (Mop 10)-Californlo Gropes: Principol Vorietics, Acreagc, ond Utilizotion, 1953} \\
\hline varitetes & Number ol acres & 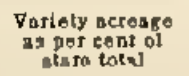 & 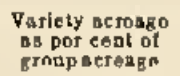 & Prinecigal atuilization \\
\hline Raisin varieties: & & & & \\
\hline Thompson Seedless. & 189,305 & 40.8 & 82.6 & Raisins, fresh, wine, and hrandy \\
\hline Muscat............... & & $\begin{array}{l}7.1 \\
0.7\end{array}$ & & $\begin{array}{l}\text { Raisins, wine, and fresh } \\
\text { Wine }\end{array}$ \\
\hline Zante Currant. & $\begin{array}{l}3,262 \\
2,262\end{array}$ & $\begin{array}{l}0.7 \\
0.5\end{array}$ & 1.0 & \\
\hline & $\begin{array}{l}2,530 \\
1,530\end{array}$ & 0.3 & 0.6 & Fresh and wine \\
\hline All rajisin varieties. & 229,228 & 49.4 & 100.0 & \\
\hline Table varieties: & & & & \\
\hline Emperor....... & 30,708 & 6.6 & & Fresh and brandy \\
\hline Tokay,......... & 24,772 & 6.4 & 28.7 & 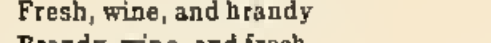 \\
\hline $\begin{array}{l}\text { Malag..................... } \\
\text { Red Milaga (Molinera). }\end{array}$ & $\begin{array}{l}8,3,30 \\
6,575\end{array}$ & $\begin{array}{l}1.8 \\
1.4\end{array}$ & $\begin{array}{l}9.7 \\
7.6\end{array}$ & $\begin{array}{l}\text { Brandy, wine, and fresh } \\
\text { Fresh and wine }\end{array}$ \\
\hline Ribier.................. & 6076 & 1.3 & 7.1 & Fresh and wine \\
\hline Cardinal. & 3,335 & 0.7 & 3.9 & \\
\hline & 466 & 0.1 & 0.6 & Fresh and juice \\
\hline others................ & 5,969 & 1.3 & 6.9 & Fresh and wine \\
\hline All table varieties. & 86,270 & 18.6 & 100.0 & \\
\hline Wine varieties: & & & & \\
\hline Zinfandel.... & 35,757 & 7.7 & 24.1 & All varieties used exclusively for wine \\
\hline Carignane ......... & 29,847 & 6.4 & 20.1 & and brandy \\
\hline Alicante Bouschet. . & 15,193 & 3.3 & 10.2 & \\
\hline $\begin{array}{l}\text { Mission...... Giden Chasselas. } \\
\text { Palomino and Golden }\end{array}$ & $\begin{array}{l}10,445 \\
10.217\end{array}$ & 2.2 & 7.0 & \\
\hline 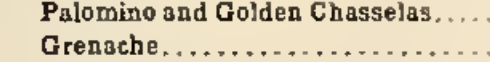 & $\begin{array}{c}10,217 \\
9,056\end{array}$ & $\begin{array}{l}2.2 \\
2.0\end{array}$ & 6.9 & \\
\hline 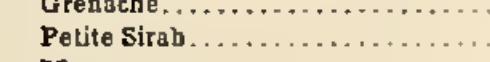 & $\begin{array}{l}9,036 \\
5,038\end{array}$ & ${ }_{1.1}^{2.0}$ & $\begin{array}{l}0.1 \\
3.4\end{array}$ & \\
\hline Mataro...... & 4,387 & 0.9 & 3.0 & \\
\hline $\begin{array}{l}\text { Burger............. } \\
\text { Saurignon Blanc. }\end{array}$ & $\begin{array}{l}3,456 \\
1739\end{array}$ & 0.7 & $\begin{array}{l}2.3 \\
2.3\end{array}$ & \\
\hline $\begin{array}{l}\text { Saunigion Blanc... } \\
\text { Colombar........ }\end{array}$ & $\begin{array}{l}1,139 \\
1,196\end{array}$ & $\begin{array}{l}0.4 \\
0.3\end{array}$ & $\begin{array}{l}1.2 \\
0.8\end{array}$ & \\
\hline Franken Riesling ..... & 966 & 0.2 & 0.6 & \\
\hline $\begin{array}{l}\text { Other white vanieties.. } \\
\text { Other red vasizties.... }\end{array}$ & 8,677 & 1.9 & 5.8 & \\
\hline $\begin{array}{l}\text { Other red dzantibes... } \\
\text { All wine varieties.. }\end{array}$ & $\begin{array}{r}14,2699 \\
148,533\end{array}$ & $\begin{array}{r}2.7 \\
32.0\end{array}$ & & \\
\hline Total _. & 464,031 & 1000.0 & 100.0 & \\
\hline
\end{tabular}

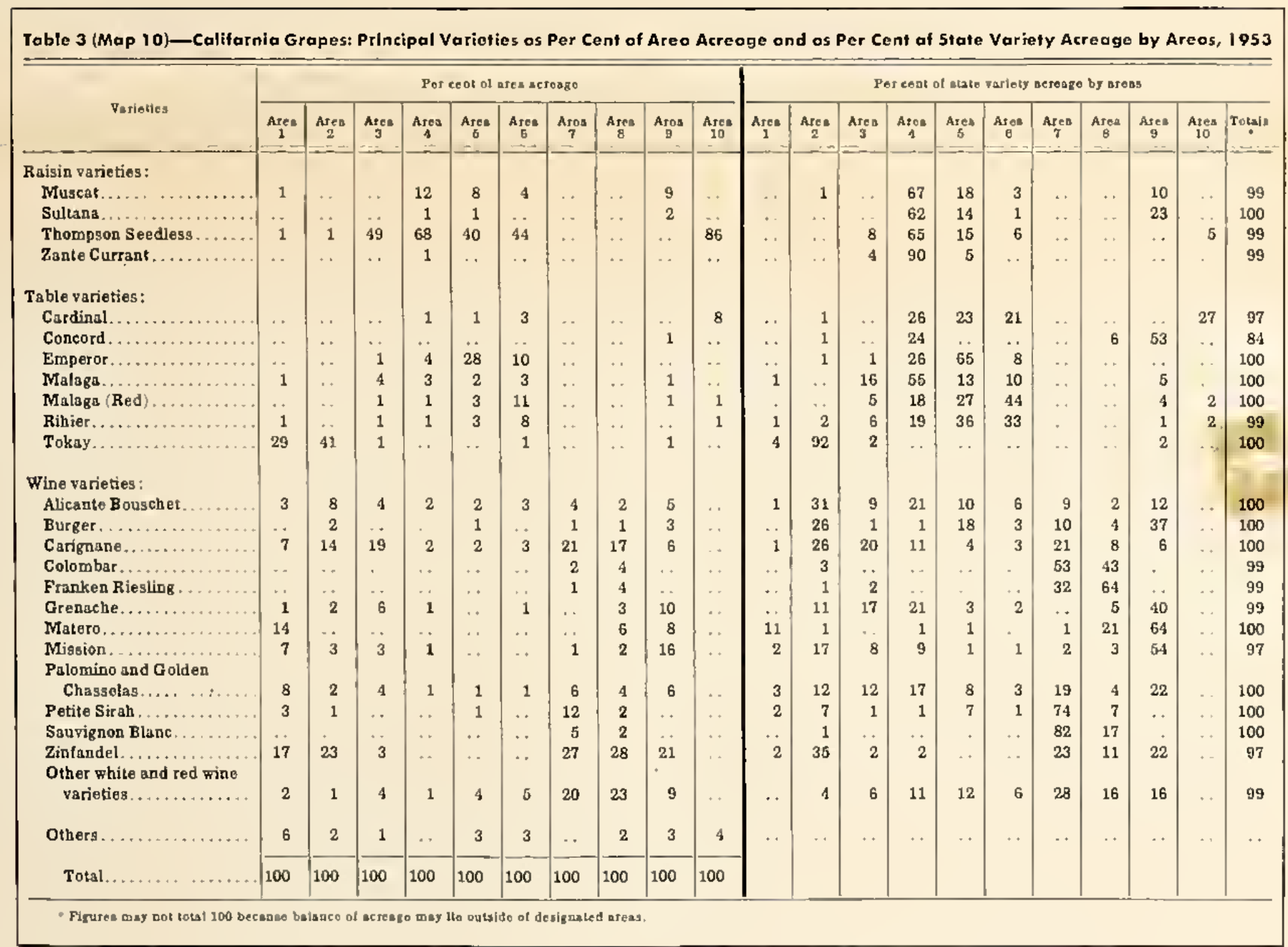

Sources of dato: Acceoge estimotes in Tables 1 ond 2 ore bosed on 1953 fotot ocreoge estimotes (beoring and nonbeor-
ingl mode by the Colifornio Crop ond tivestock Reporting Service, Socromento. Toble 3 wos compiled from unpub. 


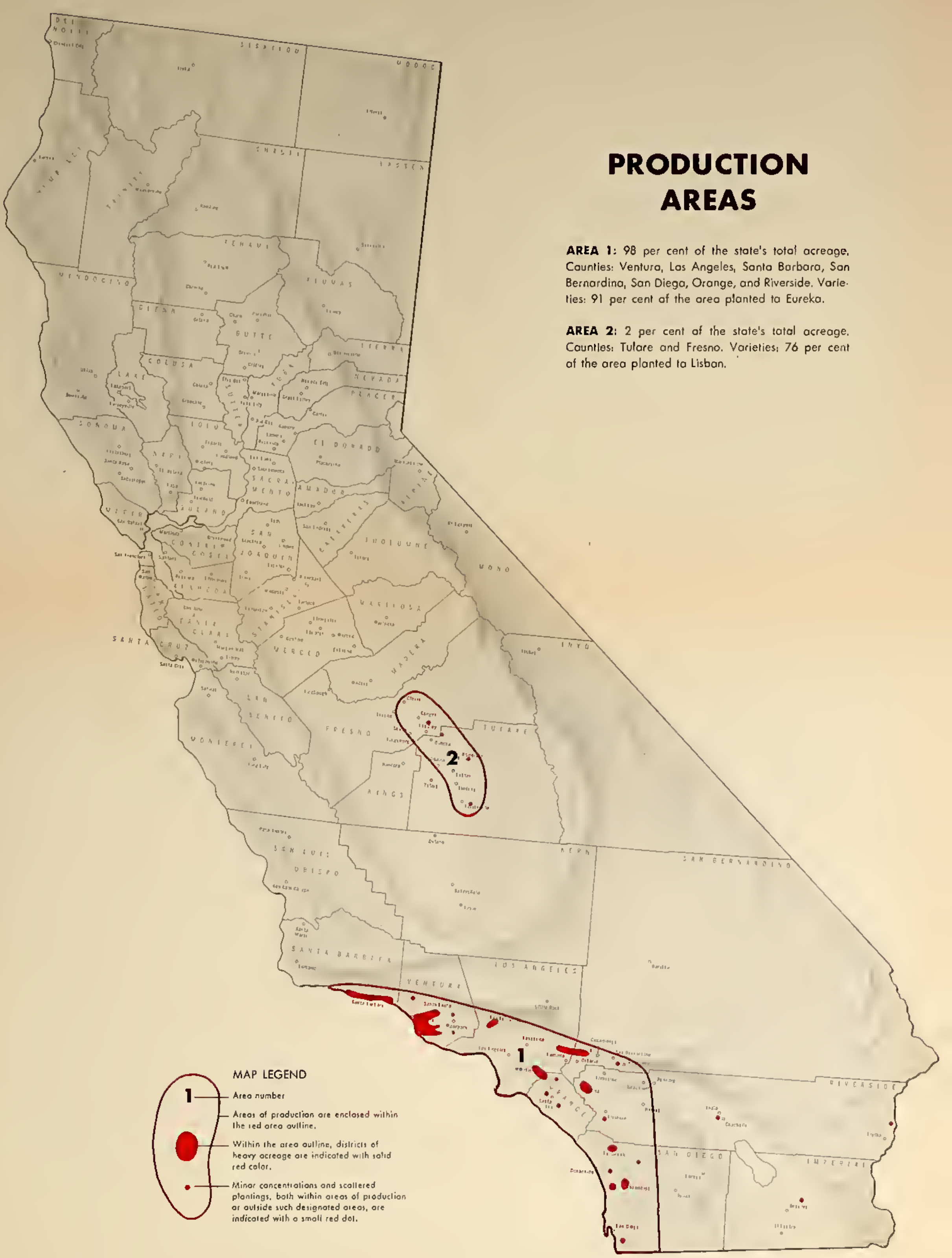

USUAL HARVEST SEASONS

\begin{tabular}{|c|c|c|c|c|c|c|c|c|c|c|c|c|}
\hline AREA AND VARIETY & JAN. & ғєв. & mer. & $A P R$. & sur & JUNE & juir & AUG. & sepr. & oct. & NOV. & DEC. \\
\hline ALL AREAS & & & & & & & & & & & & \\
\hline \\
\hline \multicolumn{13}{|c|}{$\begin{array}{l}\text { Ventura ond } \\
\text { Santa Barbara caunties }\end{array}$} \\
\hline \multicolumn{13}{|c|}{$\begin{array}{l}\text { Las Angeles and } \\
\text { San Bernordina counties }\end{array}$} \\
\hline & & & & & & & & & & & & \\
\hline & & & & & & & & & & & & \\
\hline
\end{tabular}

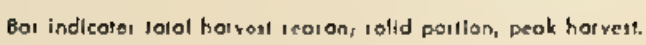




\begin{tabular}{|c|c|}
\hline \multicolumn{2}{|c|}{$\begin{array}{l}\text { Table I (Mop 11)-Califarnio Lemons: Tołol Acreaga (8earing and } \\
\text { Nonbearing) by Areos ond Cauntles, } 1953\end{array}$} \\
\hline Ares and eonnty & Number of actes \\
\hline Area 1-Southem Californis: & \\
\hline Los Angoles................. & $\begin{array}{l}9,561 \\
4,771\end{array}$ \\
\hline $\begin{array}{l}\text { Orange.......... } \\
\text { Riverside...... }\end{array}$ & $\begin{array}{l}4,771 \\
3,275\end{array}$ \\
\hline San Bernardino. & 5,632 \\
\hline San Diego......... & 4,840 \\
\hline $\begin{array}{l}\text { Sants Berbara. } \\
\text { Ventura....... }\end{array}$ & 9,239 \\
\hline Ventura....... & 21,992 \\
\hline Ares total... & . 59,310 \\
\hline Area 2-San Josquín Valley: & \\
\hline $\begin{array}{l}\text { Fresno } \ldots \ldots \ldots \\
\text { Tulare......... }\end{array}$ & $\begin{array}{r}196 \\
1,107\end{array}$ \\
\hline Area total. . & 1,303 \\
\hline Additional counties total. . & 132 \\
\hline State total. . & 60,745 \\
\hline
\end{tabular}

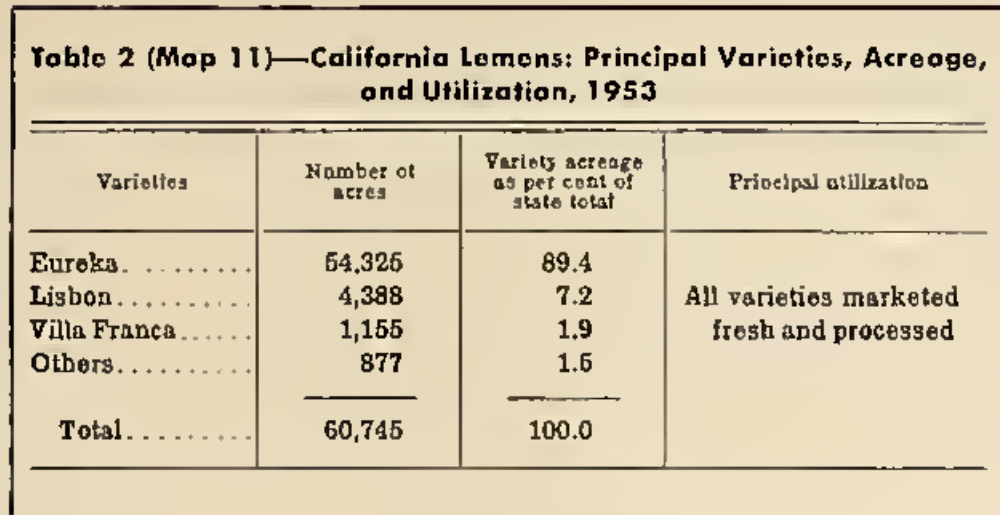

Sources of doto: Acreage estimotes in Tables 1 and 2 ore bosed on 1953 totol acreage estimates (beoring and nonbeor ing) mode by the Colifornio Crop and Livestock Reporting Service, Socromento. 


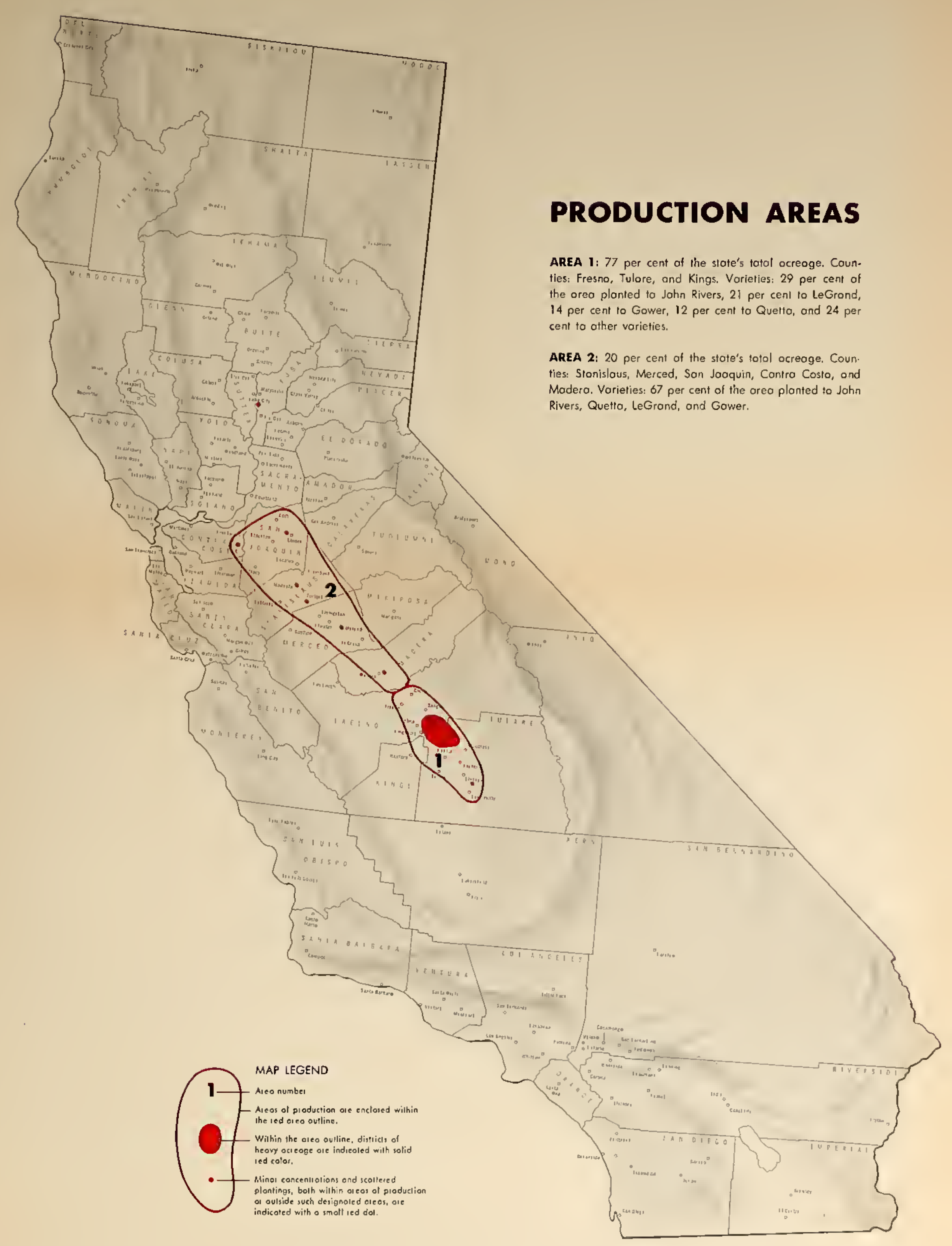

USUAL HARVEST SEASONS

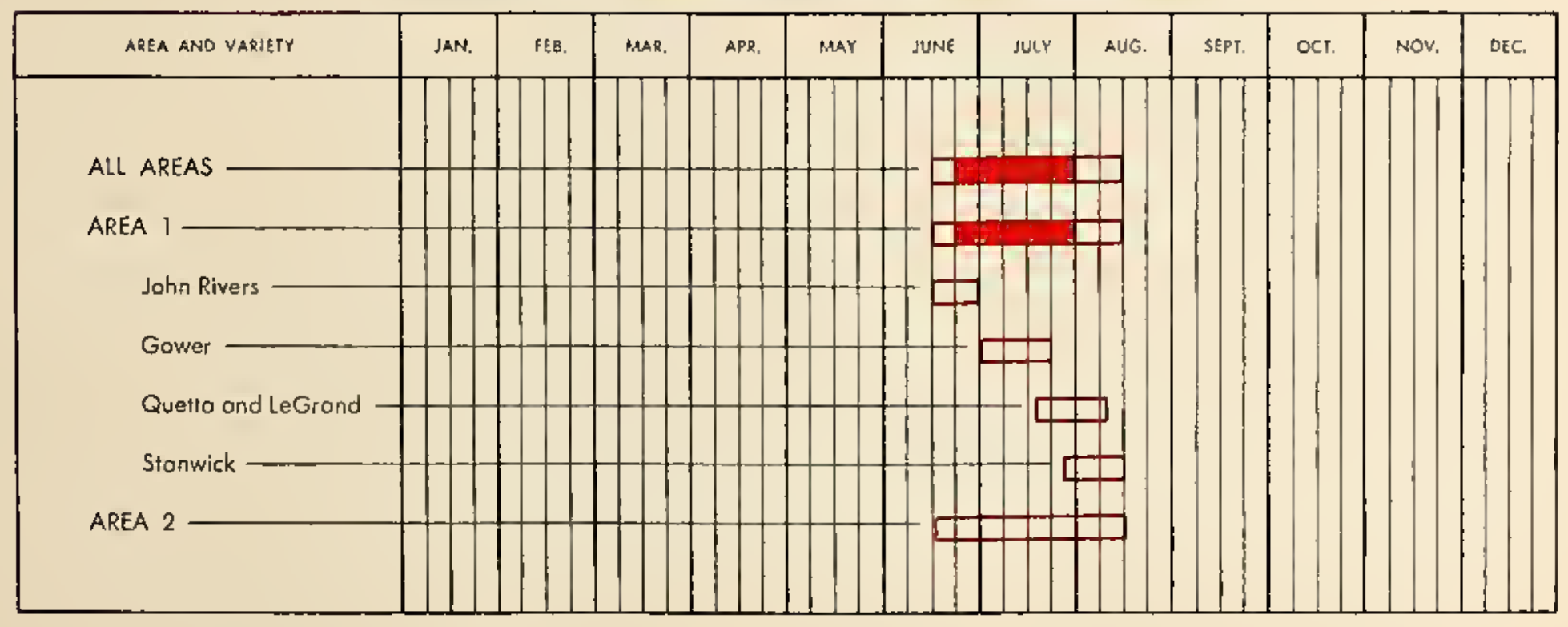

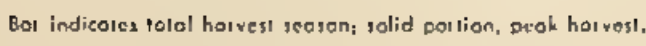




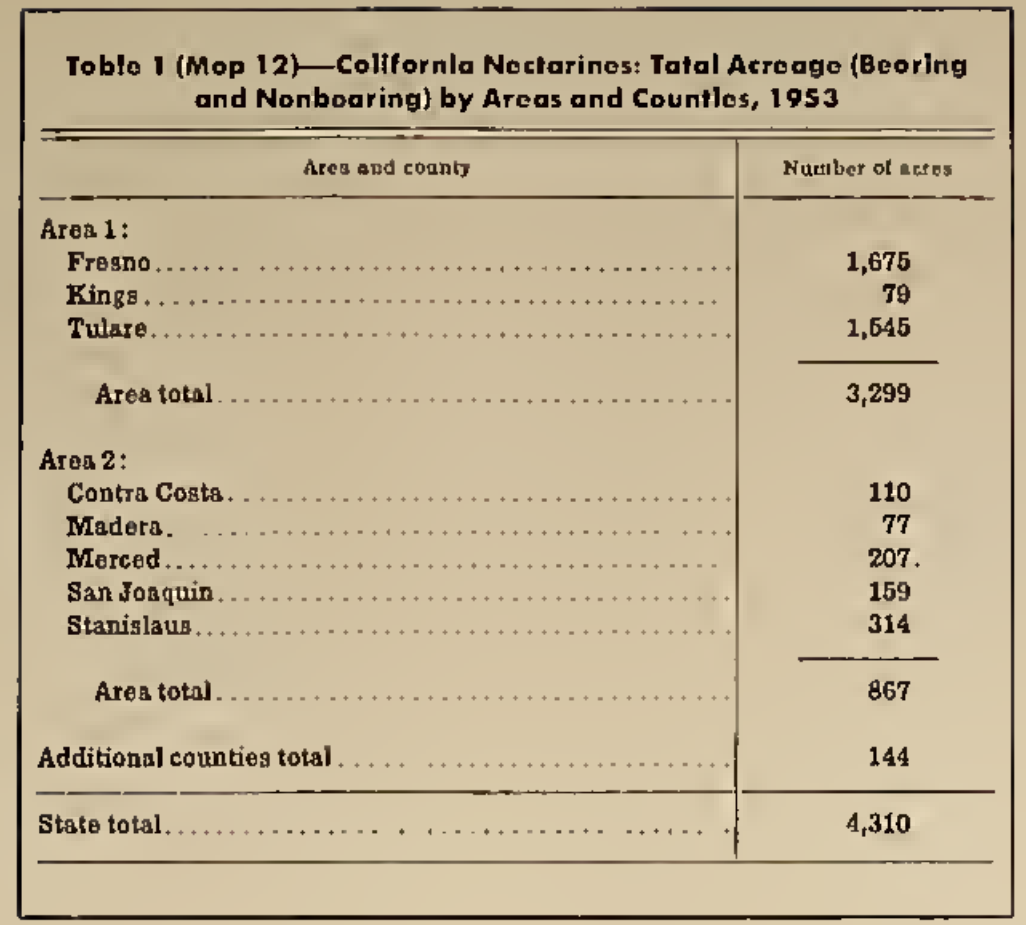

Tablo 2 (Mop 12)-Colifornia Nectarines: Principal Vorietles, Acreoge, ond

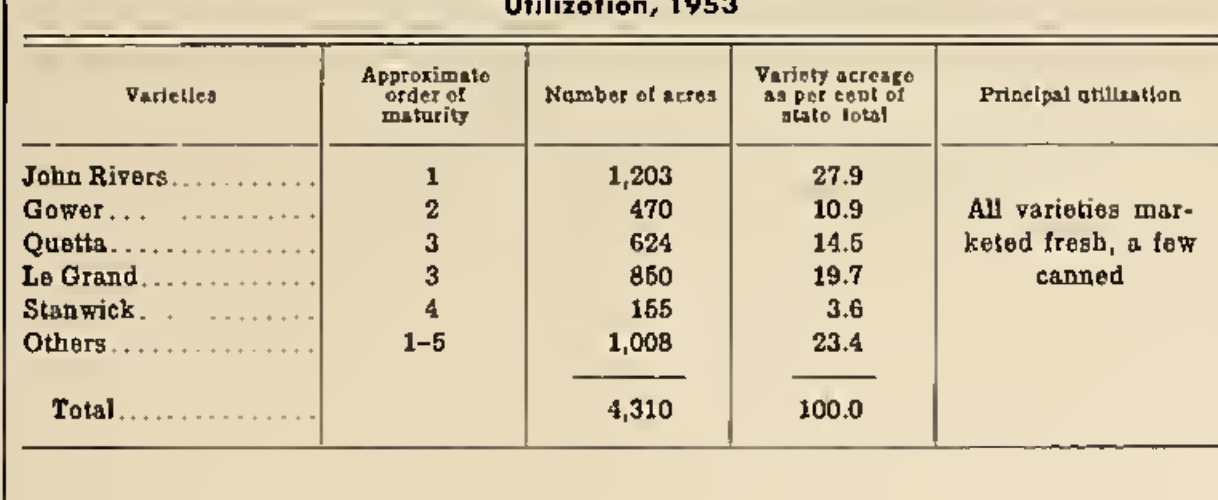

Sources of dalo: Acreoge estimates in Tables 9 ond 2 ore bosed on 1953 totol acreoge estimates (beoring ond nonbeo ing) mode by the Colitornia Crop and Livestack Reporting Service, Socromento. 
MAP 13 - PRODUCTION AREAS AND HARVEST SEASONS FOR CALIFORNIA

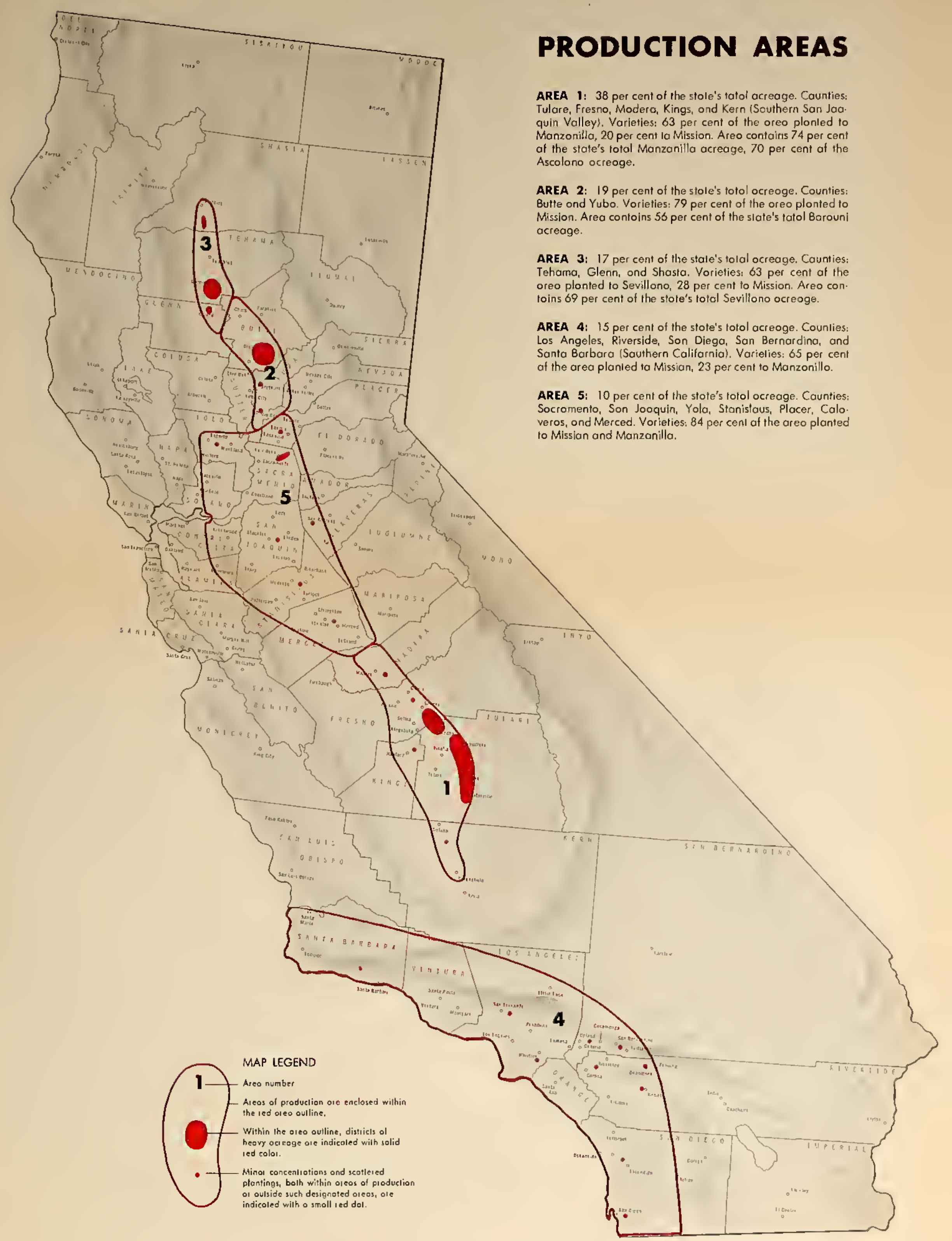

\section{USUAL HARVEST SEASONS}

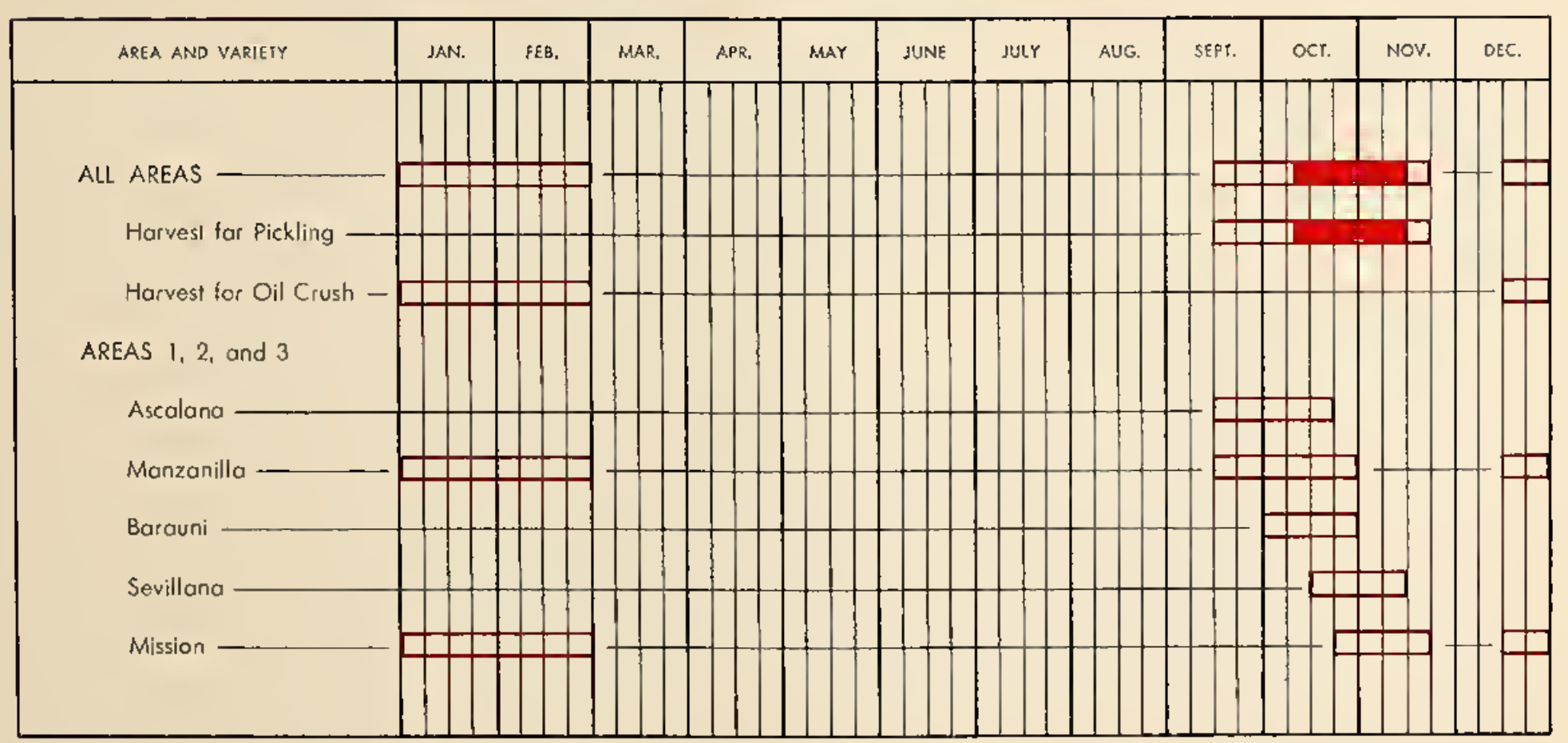

Eol indicoles lotol halvesl scoson; solio ponl lion, peok horesest. 


\begin{tabular}{|c|r||r|r|}
\hline \multicolumn{3}{|c|}{ Tablo I (Map 13)-Callfarnia Olives: Tatal Acreage (Bearling and Nonbearing) } \\
by Areas and Cauntles, 1953
\end{tabular}

\begin{tabular}{|c|c|c|c|c|}
\hline \multicolumn{5}{|c|}{ Tabie 2 (Map 13)-Califarnia Olives: Principal Varieties, Maturity, Acreage, } \\
and Utilizatlan, 1953
\end{tabular}

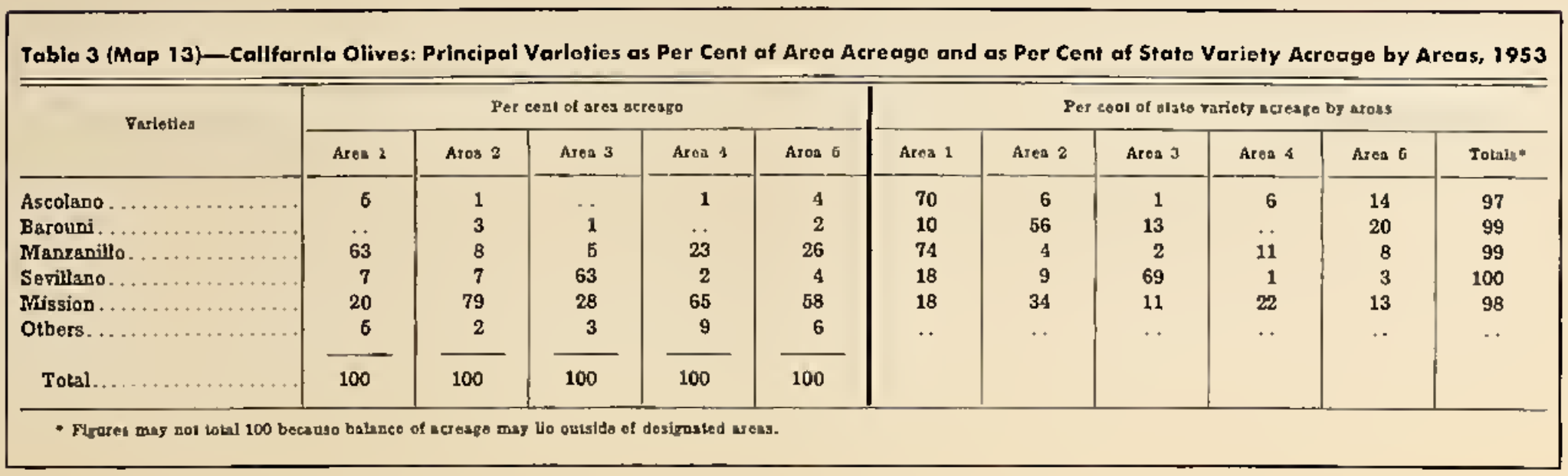

Sources of dala: Acreage estimater in Tobles 1 and 2 are based on 1953 latal acreage estimates (bearing ond nanbearing) made by the Colifornio Crop and Livestock Reporting Service, Sacromento. Table 3 was compiled fram unpub- 


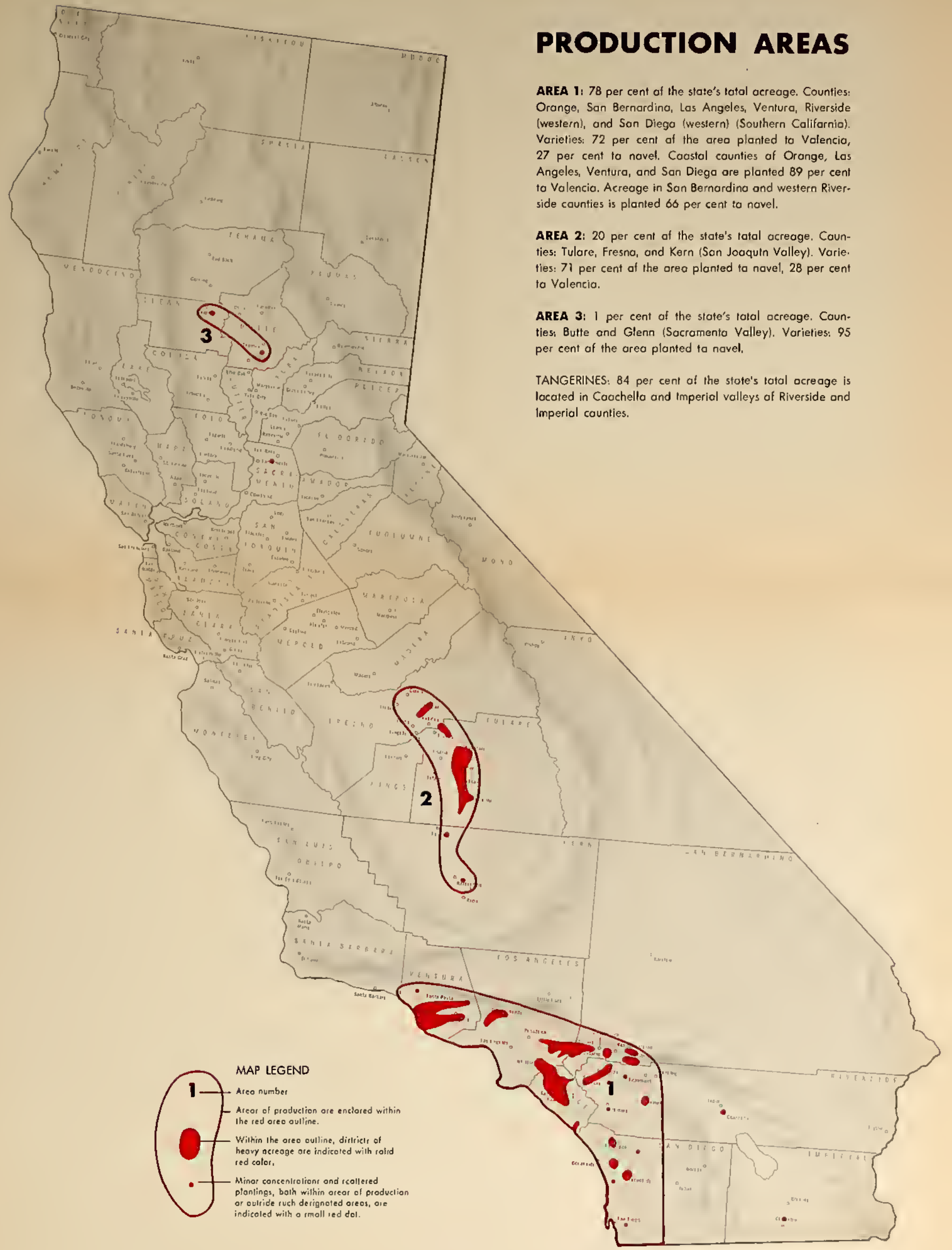

\section{USUAL HARVEST SEASONS}

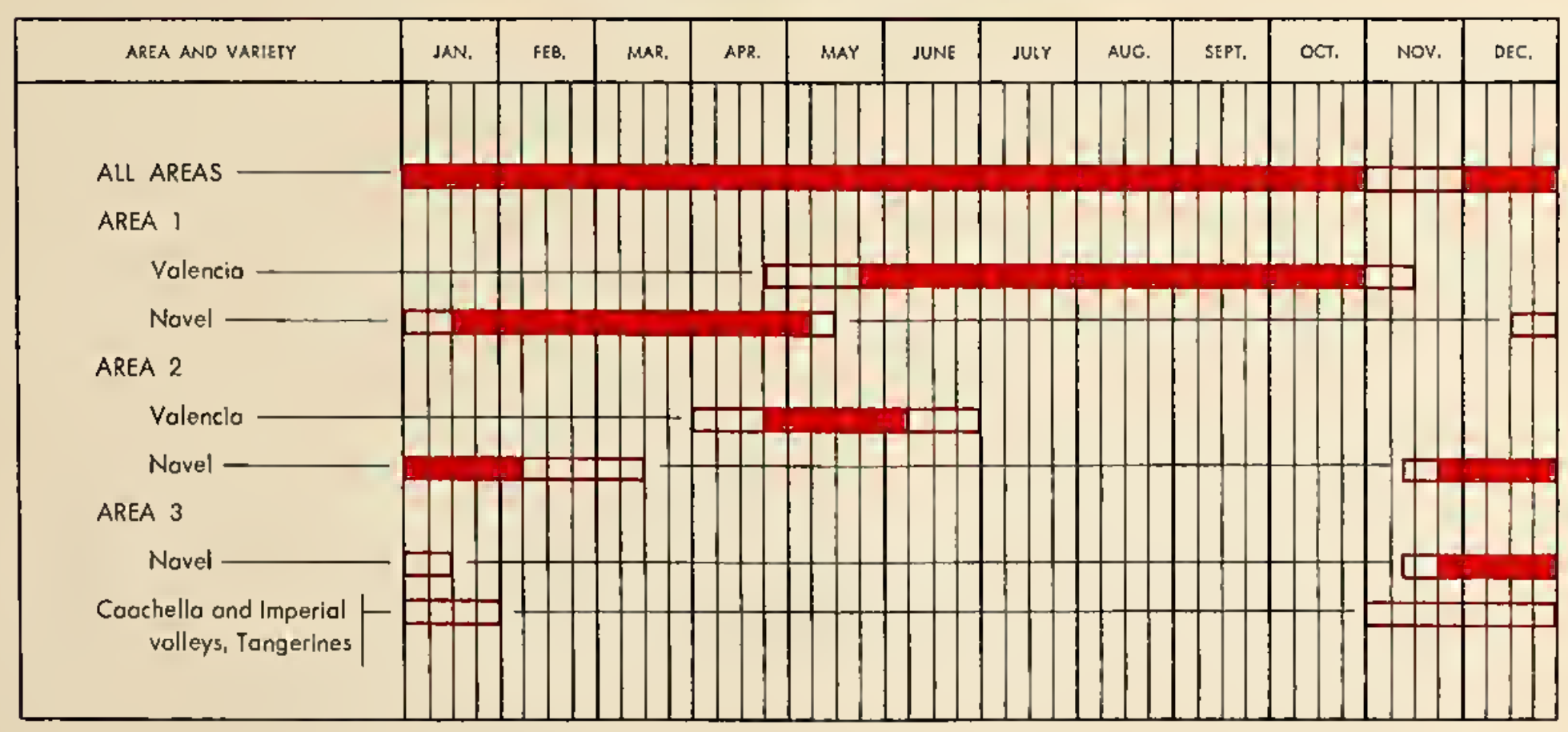




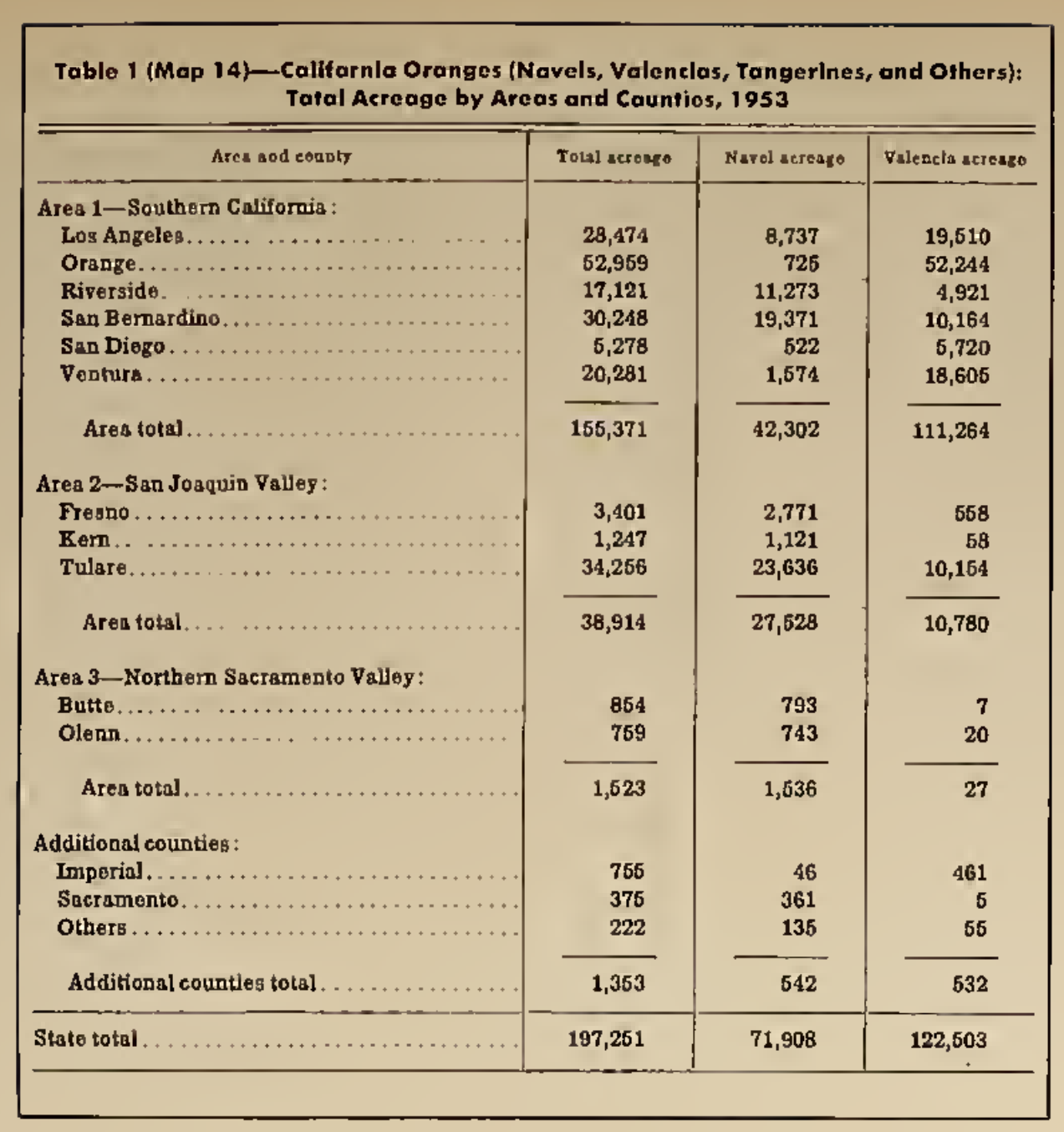

\begin{tabular}{|c|l|c|c|c|}
\hline \multicolumn{5}{|c|}{ Table 2 (Map 14)—Califarnla Oranges: Principal Varloties, Maturity, Acreage, } \\
and Utilizatian, 1953
\end{tabular}


MAP 15 - PRODUCTION AREAS AND HARVEST SEASONS FOR CALIFORNIA
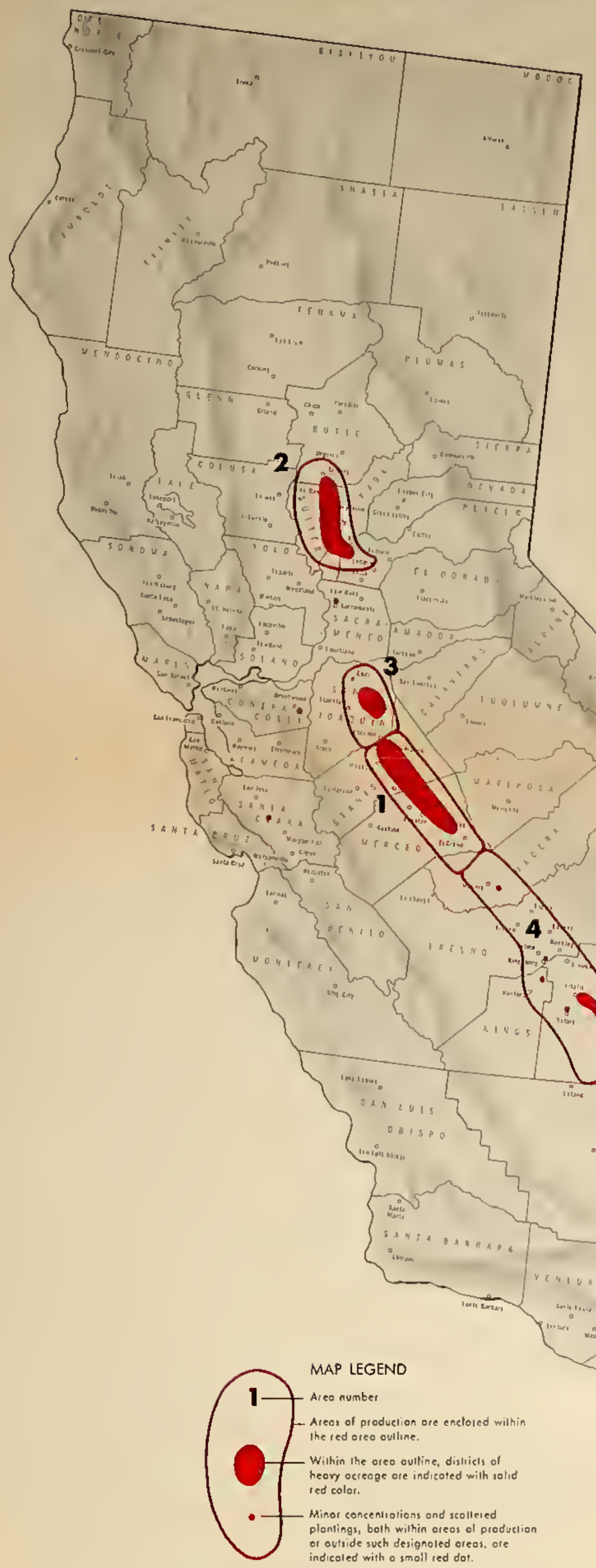

USUAL HARVEST SEASONS

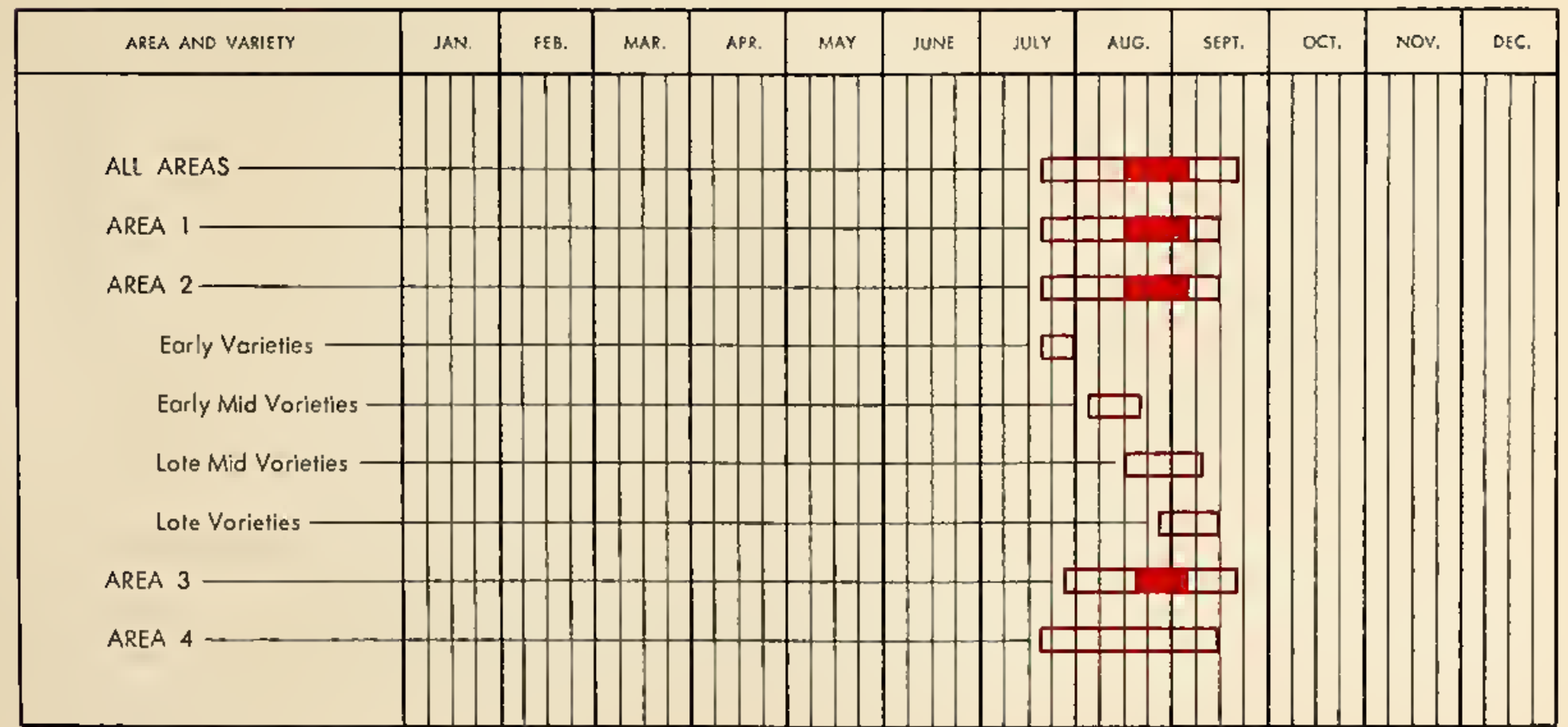
NOTE, Eorly vorieties include Fortuno ond Shosto; eorly mid vorieties, Cortez, Walton Poloro, Peok, Johnson, ond Andoro; lote mid vorieties, Goume,
Libbee, Sims, Sullivon \#1, Corolyn, Stonford, Holford, ond Willioms; lote vorieties, Philfips Cling, Stuort.Gomes, Wiser, Giblin, Corono, ond Levi.

\section{PRODUCTION AREAS}

AREA 1: 37 per cent of the stote's totol acreage. Coun. ties: Slanislaus and Merced. Varielies: 75 per cent af the Games, Peak, and Cartez.

AREA 2: 37 per cent ol the state's total acreage. Caunties: Sutter, Yubo, Butte, and Placer. Varieties: 76 per cent Sims, Corolyn, Palara, Cortez, ond Fortuno.

AREA 3: 13 per cent af the stole's lotol acreage. County San Joaquin. Vorieties: 66 per cent of the orea planted to Halfard, Palara, Gaume, ond Stuart.Games.

AREA 4: 9 per cent al the state's total ocreoge. Counries: Tulare, Kings, Fresna, and Madera. Varieties: 55 per Fartuna. 


\begin{tabular}{|c|c|c|c|}
\hline \multicolumn{4}{|c|}{$\begin{array}{c}\text { Table } 1 \text { (Map 15)-Callfarnio Clingstóne Peoches: Tatal Acreage (Bearing and } \\
\text { Nonbearing) by Areas and Counties, 1953 }\end{array}$} \\
\hline Area and county & Number of acroa & Area and county & Nambor of acres \\
\hline $\begin{array}{l}\text { Ares 1: } \\
\quad \text { Merced.... }\end{array}$ & 4,143 & $\begin{array}{l}\text { Ares 4: } \\
\text { Fresno.. }\end{array}$ & 496 \\
\hline Stanislaus. & 17,004 & $\begin{array}{l}\text { Madera } \\
\text { Kingo }\end{array}$ & 465 \\
\hline Ares total. . & 21,147 & Tulare... & $\begin{array}{l}3,804 \\
3,846\end{array}$ \\
\hline Ares 2: & & Ares total.. & 5,360 \\
\hline Butte.. & 2,713 & & \\
\hline Placer. & $\begin{array}{r}635 \\
1438\end{array}$ & $\begin{array}{l}\text { Additional counties : } \\
\text { Sng Berpardino }\end{array}$ & \\
\hline $\begin{array}{l}\text { Sutter.. } \\
\text { Tuth }\end{array}$ & 14,348 & San Bernardino ... & $\begin{array}{l}668 \\
381\end{array}$ \\
\hline Yuba... & 3,546 & $\begin{array}{l}\text { Riverside....... } \\
\text { Sacramnto.... }\end{array}$ & $\begin{array}{l}381 \\
225\end{array}$ \\
\hline Ares total. & 21,142 & Santa Clara... & $\begin{array}{l}129 \\
105\end{array}$ \\
\hline $\begin{array}{l}\text { Ares 3: } \\
\text { An }\end{array}$ & 7,344 & Additionsl counties totsl. & 1,698 \\
\hline Area total & 7,344 & State total....... & 56,691 \\
\hline & & & \\
\hline
\end{tabular}

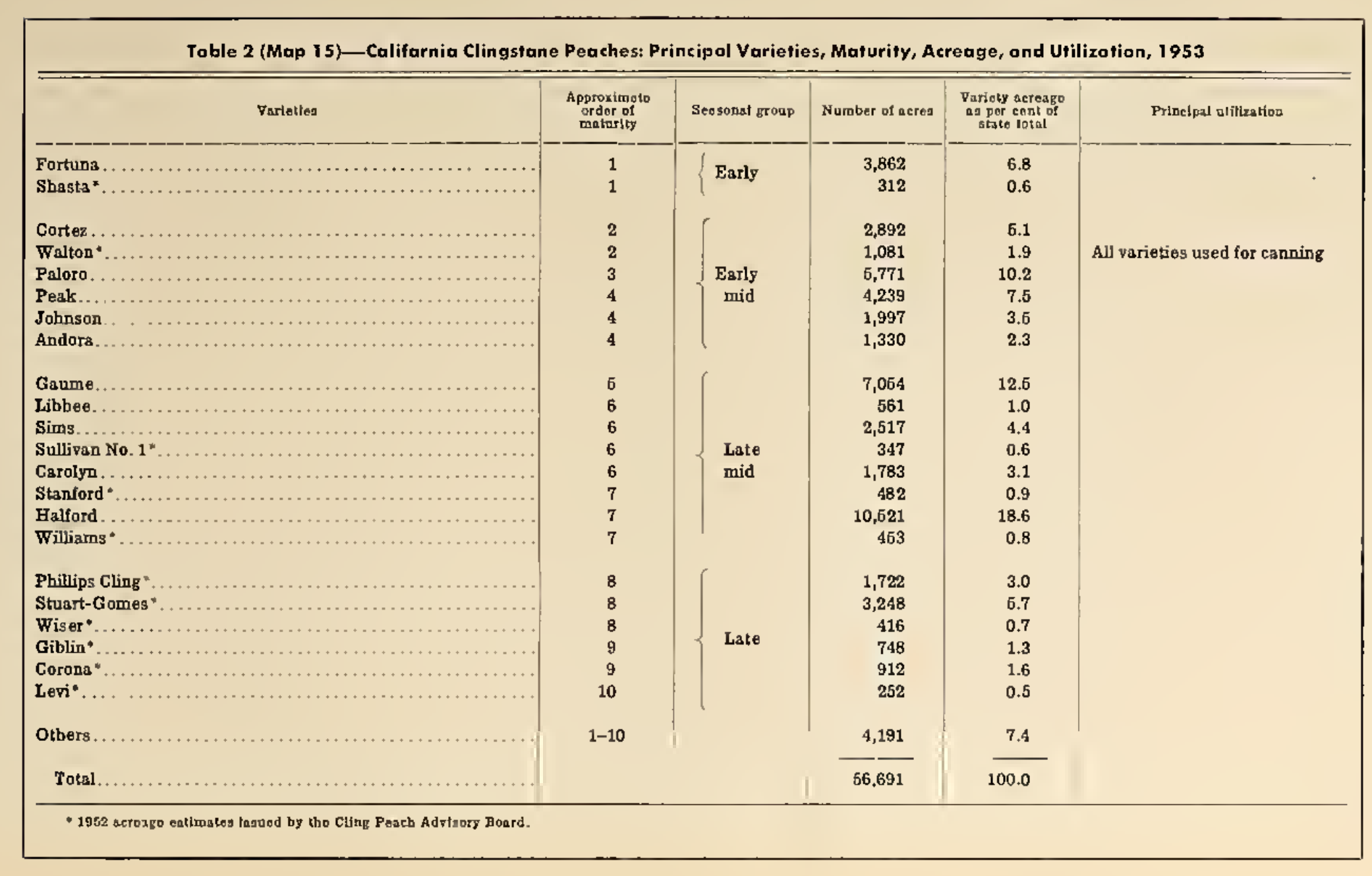

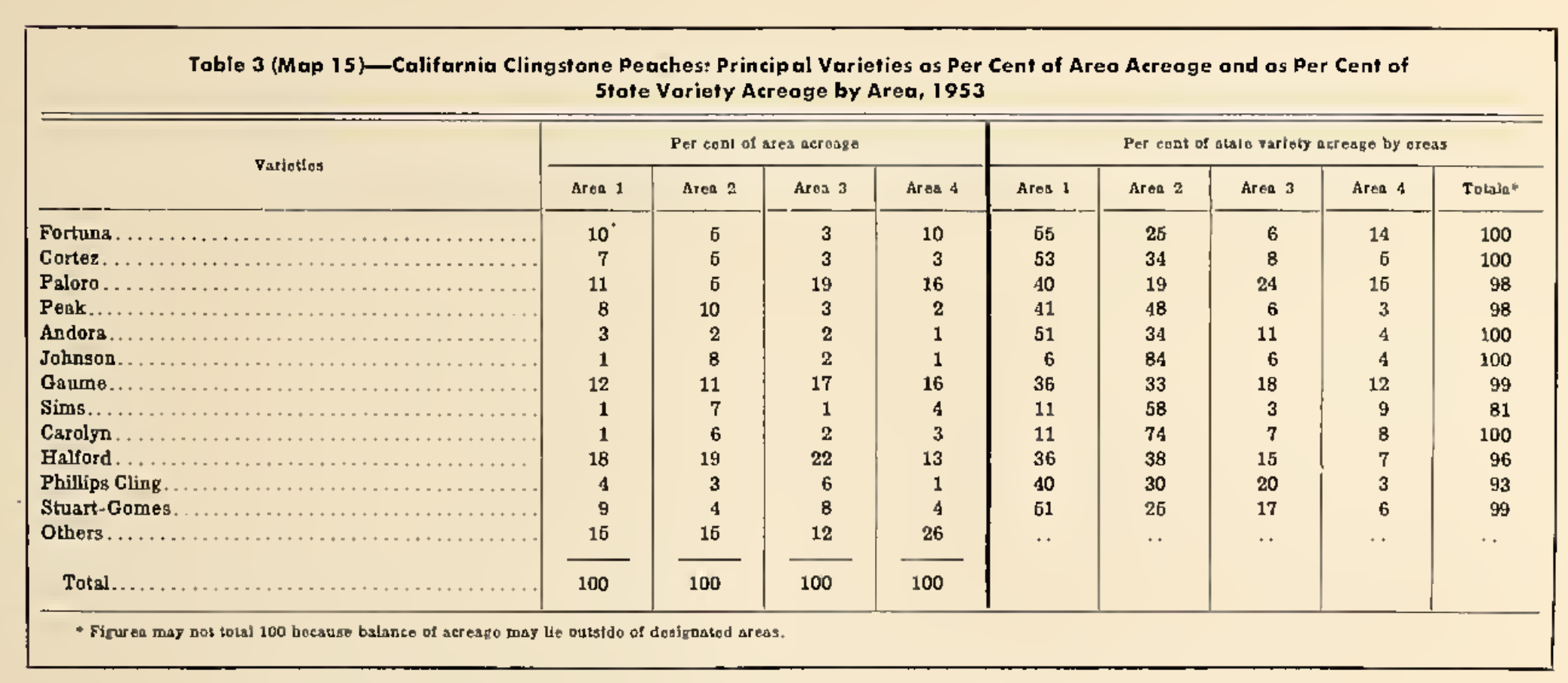

Sources of dato: Arreoge estimotes in Tabtes 1 and 2 are bosed on 1953 potol ocreoge estimotes (beoring ond nonbeor. ing) mode by the Colifirnio Crop ond livestock Reporting Service, Sacromento. Toble 3 wos compiled from unpub.
lished dota of the sime ogencry. 
MAP 16 - PRODUCTION AREAS AND HARVEST SEASONS FOR CALIFORNIA

\section{FREESTONE PEACHES}

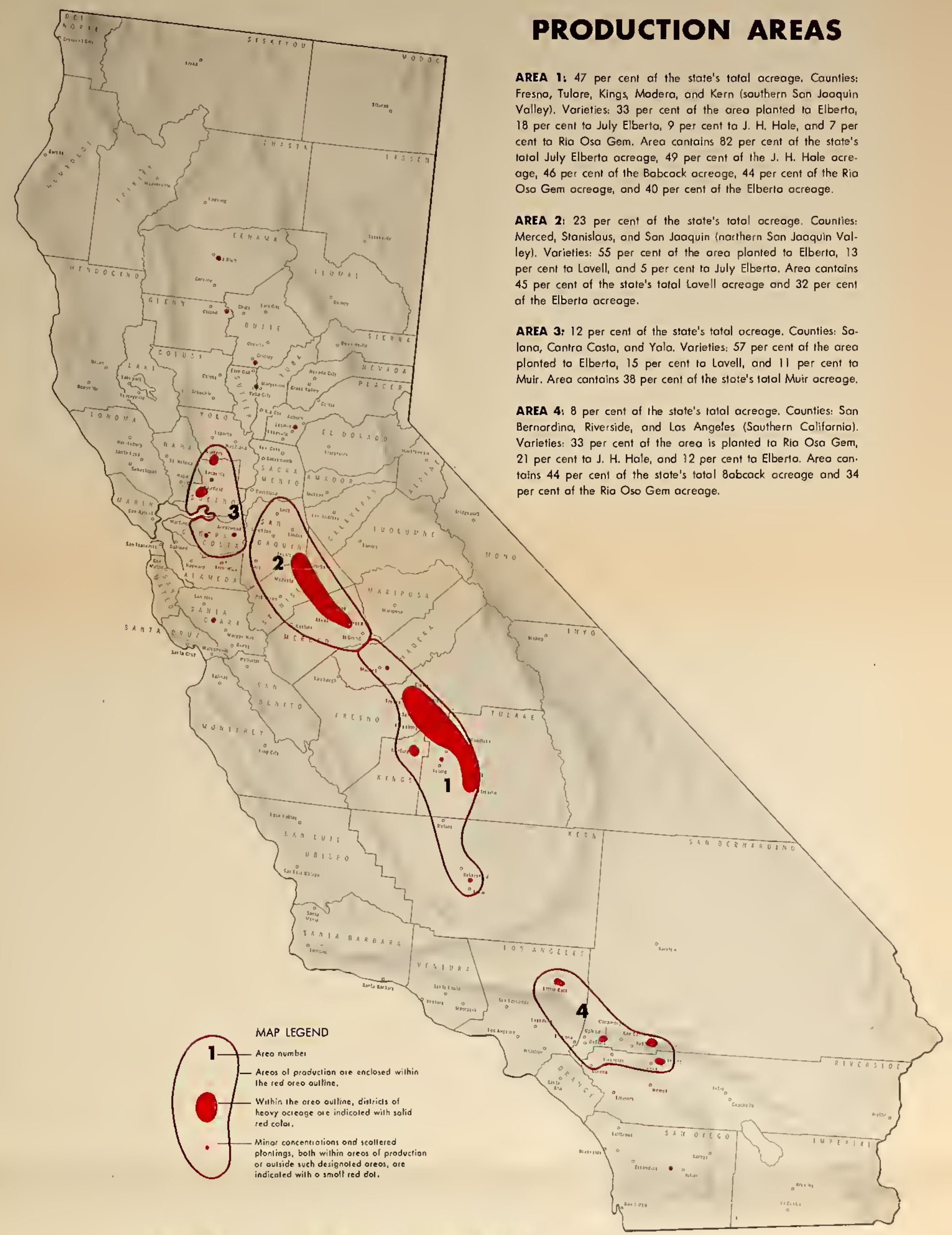

USUAL HARVEST SEASONS

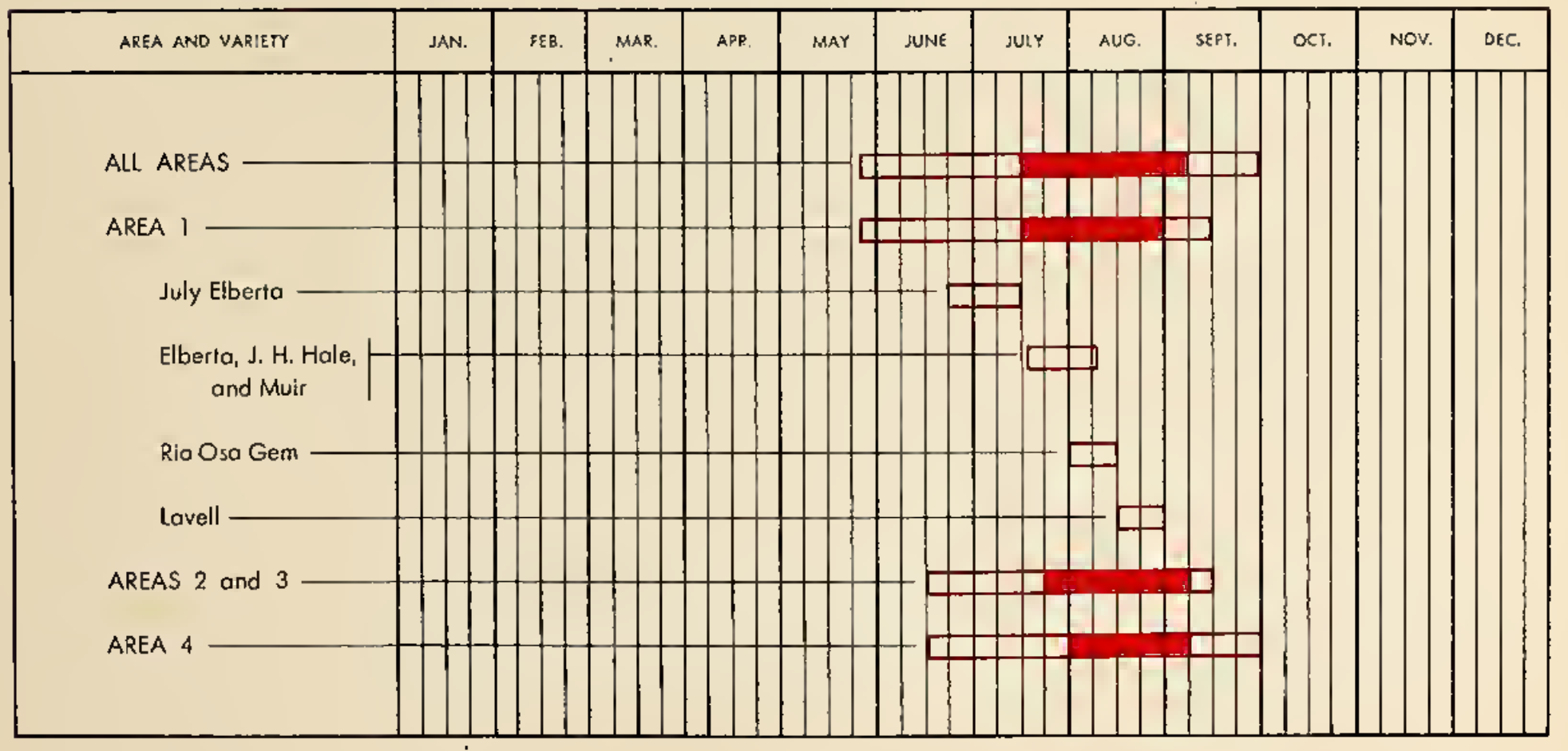

Bor indicotes lotol horvess seosons solld portion peok harvest. 


\begin{tabular}{|c|c|c|c|}
\hline \multicolumn{4}{|c|}{$\begin{array}{l}\text { Table I (Mop 16)-Californla Freestane Peachess: Totol Acreage (Bearing and } \\
\text { Nonbeoring) by Areas and Countios, 1953 }\end{array}$} \\
\hline Area and ceuniy & Number of secos & Aros snd county & Namber of acres \\
\hline $\begin{array}{l}\text { Ares 1-Southern San } \\
\text { Josquin Volloy: }\end{array}$ & & Ares 4-Southern Californin: & \\
\hline Fresso ............. & 9,908 & $\begin{array}{l}\text { Riverside. . . . } \ldots \ldots \ldots \ldots \ldots \ldots \\
\end{array}$ & 1,126 \\
\hline Kern ... & 191 & San Bernardino. & 1,307 \\
\hline Kings... & 1,336 & & \\
\hline Madera. & 1,043 & Ares total... & 3,227 \\
\hline Tulare.. & 6,081 & Addiltional counties: & \\
\hline Area total. & 18,569 & Tehuma........ & 879 \\
\hline & & Placer....... & 668 \\
\hline Area 2-Northern $S_{a n}$ & & San Diego. & 427 \\
\hline Josquin Valley: & & Butte...... & 407 \\
\hline Merced. . . . . . . & 3,373 & Sutter....... & 290 \\
\hline San Joaquin. & 2.451 & Sarts Clara. & 170 \\
\hline Stanislaus. . & 3.065 & $\begin{array}{l}\text { Olens...... } \\
\text { Others" }\end{array}$ & $\begin{array}{l}136 \\
746\end{array}$ \\
\hline Ares total. & 8,889 & & 3.723 \\
\hline Area 3: & & & \\
\hline Contra Costa. & 903 & State total. . & 38,960 \\
\hline $\begin{array}{l}\text { Solano... } \\
\text { Yolo... }\end{array}$ & $\begin{array}{r}3,144 \\
615\end{array}$ & & \\
\hline Area total. . & 4,662 & & \\
\hline
\end{tabular}

\begin{tabular}{|c|c|c|c|c|}
\hline Variotlce & 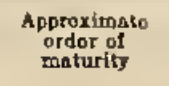 & Number of acroe & 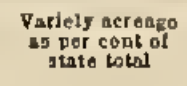 & Prtncipast niltizalion \\
\hline 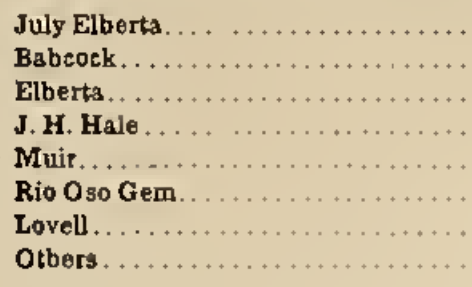 & $\begin{array}{c}3 \\
4 \\
6 \\
6 \\
6 \\
7 \\
8 \\
1-10\end{array}$ & $\begin{array}{c}4,032 \\
627 \\
16,461^{*} \\
3,285 \\
1,339 \\
3,055 \\
2,604 \\
8,664\end{array}$ & $\begin{array}{r}10.4 \\
1.4 \\
39.7 \\
8.4 \\
3.4 \\
7.8 \\
6.7 \\
22.2\end{array}$ & $\begin{array}{l}\text { Fresh market; a few canned and dried } \\
\text { Fresb market } \\
\text { Frest market, canned; ; few dried and frozen } \\
\text { Fresh markat, canned, and frozen } \\
\text { Dried } \\
\text { Fresh market, frozen; ; few canned } \\
\text { Dried } \\
\text { Fresh market }\end{array}$ \\
\hline Total.. & & 38,960 & 100.0 & \\
\hline
\end{tabular}

\begin{tabular}{|c|c|c|c|c|c|c|c|c|c|}
\hline \multirow{3}{*}{ Vartelion } & $\begin{array}{l}\text { Tone Pec } \\
\text { Stat }\end{array}$ & $\begin{array}{l}\text { es: Pring } \\
\text { oriety A }\end{array}$ & $\begin{array}{l}\text { o Vorief } \\
\text { age by }\end{array}$ & $\begin{array}{l}\text { os Per } \\
05,195\end{array}$ & it of Are & Acreage & d os Per & int of & \\
\hline & \multicolumn{4}{|c|}{ Per cenl of area acroago } & \multicolumn{5}{|c|}{ Per cenl of taslo variety acreage by arean } \\
\hline & Area 1 & Area 2 & Area 3 & Aren 4 & Aros 2 & Area 2 & Ares 3 & Arca 1 & Tolatsa \\
\hline \multirow{2}{*}{$\begin{array}{l}\text { July Elberta. . } \\
\text { Babcock..... }\end{array}$} & 18 & 5 & . & 4 & 82 & 10 & .. & 3 & 95 \\
\hline & 1 & & & 7 & 46 & 2 & & 44 & 92 \\
\hline Elbertis........ & 33 & 55 & 57 & 12 & 40 & 32 & 17 & 3 & 92 \\
\hline \multirow{2}{*}{$\begin{array}{l}\text { J. H. Hale . . } \\
\text { Muir }\end{array}$} & 9 & 4 & 9 & 21 & 49 & 12 & 12 & 20 & 93 \\
\hline & 2 & 4 & 11 & & 27 & 28 & 38 & & 93 \\
\hline $\begin{array}{l}\text { Muir.......... } \\
\text { Rio Oso Gem. }\end{array}$ & 7 & 4 & 2 & 33 & 44 & 12 & 3 & 34 & 93 \\
\hline \multirow{2}{*}{$\begin{array}{l}\text { Lovell......... } \\
\text { Others....... }\end{array}$} & 3 & 13 & 15 & & 22 & 45 & 27 & 1 & 95 \\
\hline & 27 & 16 & 6 & 23 & & .. & .. & & \\
\hline Total......... & 100 & 100 & 100 & 100 & & & & & \\
\hline
\end{tabular}

Sources of dalo: Acreage estimoles in Tobles 1 and 2 are based an 1953 todol acreage estimates (bearing ond nanbearing) made by the Colitionio Crop and livestack Reparting Serviec. Sacramento. Tabte 3 was compiled from unput thed dato of the same ogento 
MAP 17 - PRODUCTION AREAS AND HARVEST SEASONS FOR CALIFORNIA

PEARS

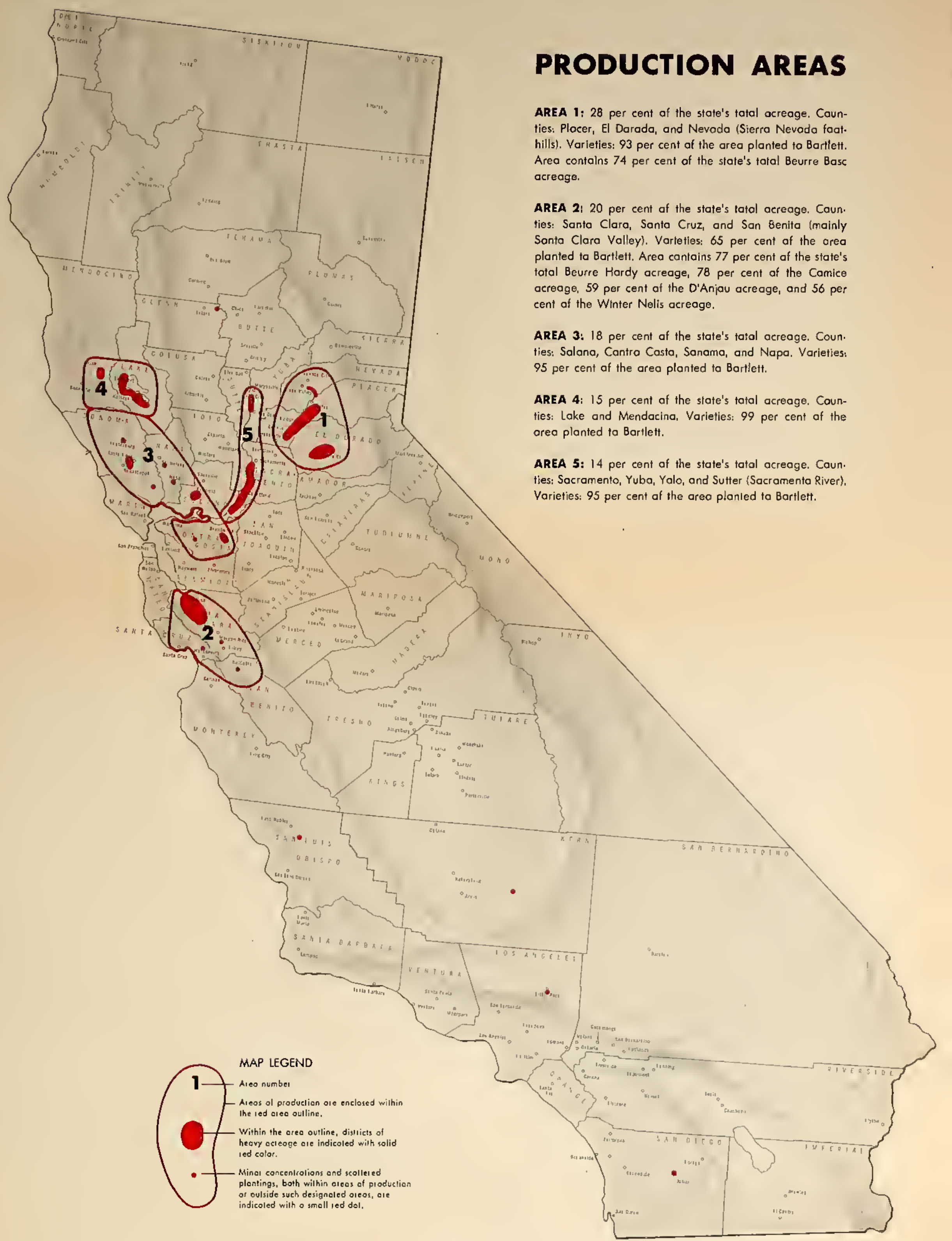

USUAL HARVEST SEASONS

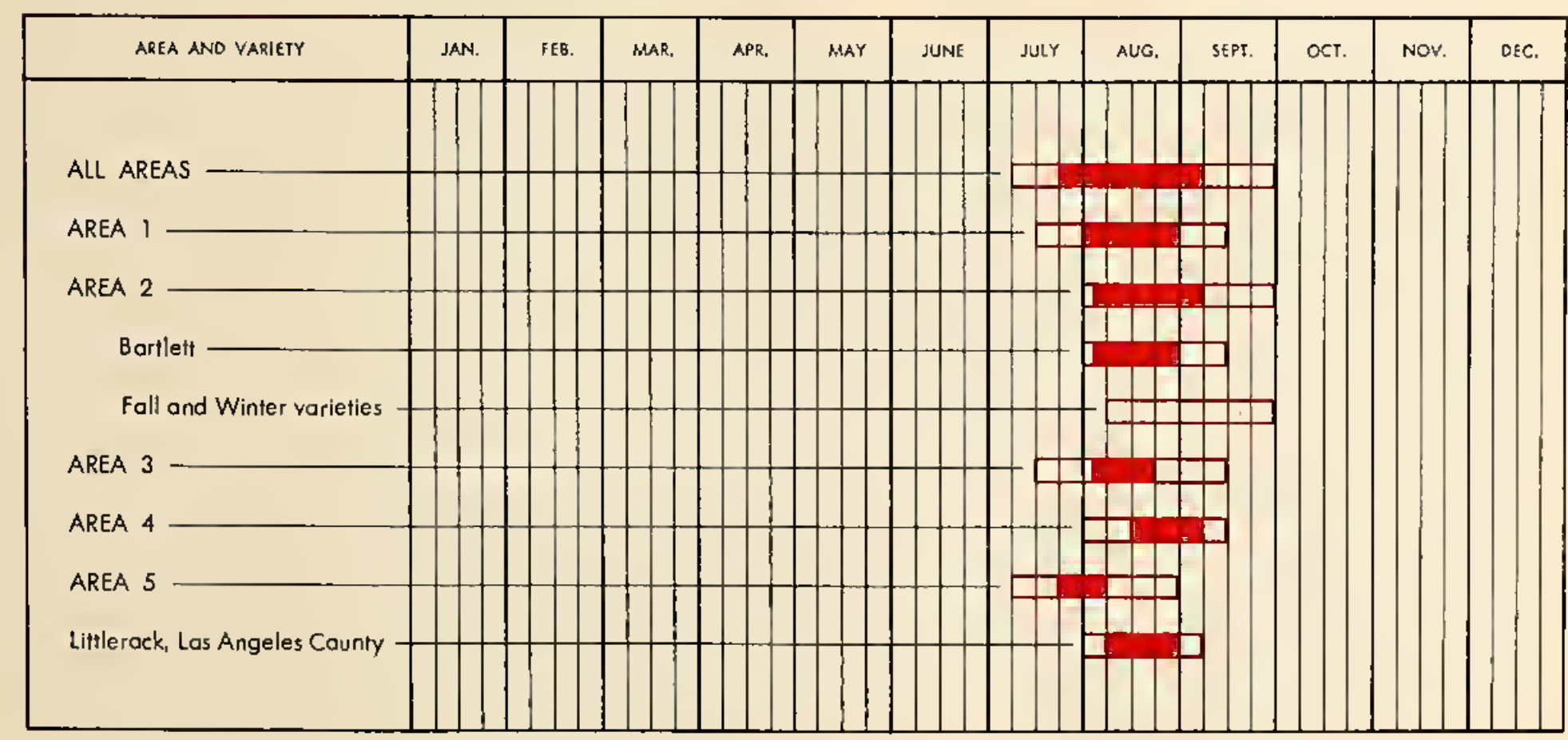

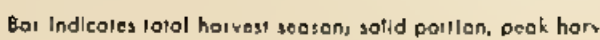




\begin{tabular}{|c|r||c|c|}
\hline \multicolumn{3}{|c|}{ Table 1 (Map 17) Callfarnla Pearss Taral Acreage (Bearlng and Nanbearlng) } \\
by Araas and Caunfies, 1953
\end{tabular}

\begin{tabular}{|c|c|c|c|}
\hline \multicolumn{4}{|c|}{ Table 2 (Map 17)-Callfarnia Pears, Principal Varioties, Acreage, and } \\
Utillyation, 1953
\end{tabular}

\begin{tabular}{|c|c|c|c|c|c|c|c|c|c|c|c|}
\hline \multirow{2}{*}{ Variotiog } & \multicolumn{5}{|c|}{ Por cent ol aroa seroago } & \multicolumn{6}{|c|}{ Por ceot of otato farioty acrosgo by aroas } \\
\hline & Ares 1 & Aros 2 & Area 3 & Area 4 & Area 5 & Ares 1 & Area 2 & Area 3 & Arra 4 & Aros 5 & Tobats" \\
\hline Bartlett... . . & 93 & 65 & 95 & 99 & 95 & 29 & 15 & 19 & 17 & 15 & 95 \\
\hline Beurre Bosc... & 3 & 1 & & & & 74 & 23 & & & & 97 \\
\hline Beurre Hвrdy. & 1 & 18 & 2 . & .. & 3 & 6 & 77 & 6 & .. & 9 & 98 \\
\hline Comice....... & & 7 & 1 & .. & .. & 5 & 78 & 11 & & & 94 \\
\hline D'Anjou...... & 1 & 2 & .. & .. & 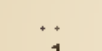 & 26 & 59 & & 10 & S & 95 \\
\hline Winter Nelis . & 1 & 5 & & & 1 & 19 & 56 & 1 & .. & 6 & 82 \\
\hline Others....... & 1 & 2 & 2 & 1 & 1 & & .. & .. & .. & & \\
\hline Total.. & 100 & 100 & 100 & 100 & 100 & & & & & & \\
\hline
\end{tabular}

Sources of dato: Acreage estimales in Tobles 1 and 2 are based on 1953 lotal acreage estimates (bearing ond nonbea ing) mode by ithe Colifornia Crap and Livestock Reporting Service. Sacramenta. Toble 3 was compiled from unpub- 


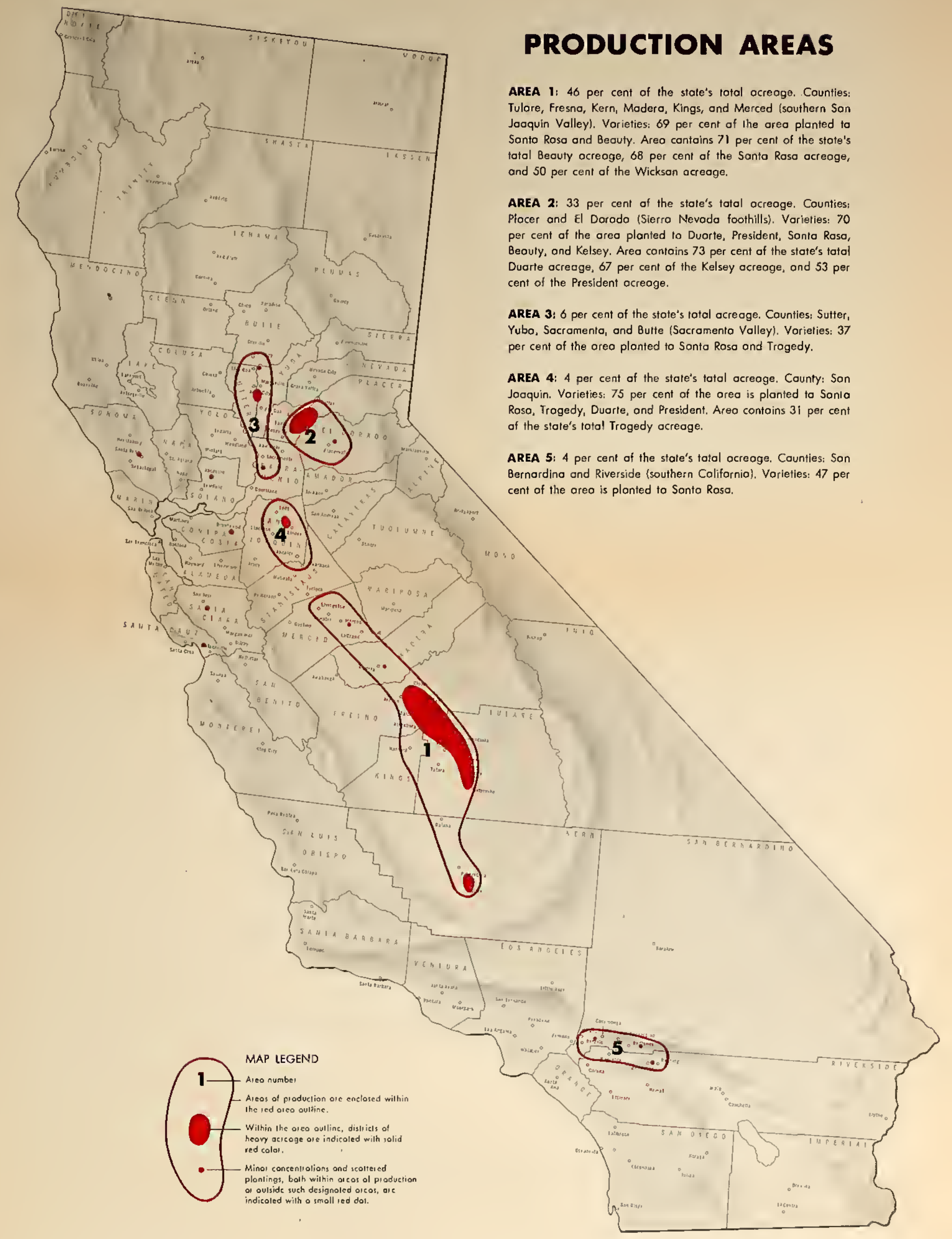

USUAL HARVEST SEASONS

\begin{tabular}{|c|c|c|c|c|c|c|c|c|c|c|c|c|}
\hline AREA ANO VARILTY & JAN. & FEB. & MAR. & APR. & MAY & JUNE & JutY & AUG. & sEPY. & ост. & Nov. & DEC. \\
\hline ALL AREAS - & & & & & & & & & & & & \\
\hline AREA $1-$ & & & & & & & & & & & & \\
\hline AREA 2 - & & & & & & & 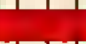 & & & & & \\
\hline AREA 3 & & & & & & & & & & & & \\
\hline AREA 4 & & & & & & & & & & & & \\
\hline AREA 5 & & & & & & & & & & & & \\
\hline & & & & & & & & & & & & \\
\hline
\end{tabular}

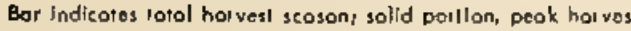




\begin{tabular}{|c|c|c|c|}
\hline Ares and conaty & Namber of aeros & Asea and connty & Nember of acros \\
\hline $\begin{array}{l}\text { Ares } 1 \text {-Souther San Joaquin Valley: } \\
\text { Fresno }\end{array}$ & 3,829 & $\begin{array}{l}\text { Area 4: } \\
\text { San Josquin.. }\end{array}$ & 984 \\
\hline & 1,808 & & \\
\hline Kings... & $\begin{array}{l}146 \\
272\end{array}$ & Ares total. . & 984 \\
\hline Madere. & $\begin{array}{l}2722 \\
130\end{array}$ & Ares 6-Southern Galifornia : & \\
\hline \multirow[t]{2}{*}{$\begin{array}{l}\text { Merced. } \\
\text { Tulare.. }\end{array}$} & 6,306 & 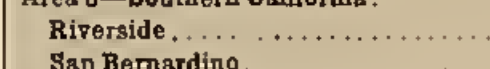 & 416 \\
\hline & 11,406 & San Bernardino. & 472 \\
\hline 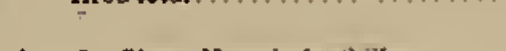 & & Area total. . & 888 \\
\hline \multirow{2}{*}{ 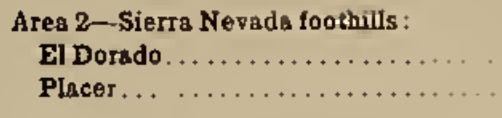 } & & Additional counties: & \\
\hline & 8,073 & $\begin{array}{l}\text { Solano............ } \\
\text { Santa Crue ...... }\end{array}$ & $\begin{array}{l}599 \\
440\end{array}$ \\
\hline Area total. & 8,234 & $\begin{array}{l}\text { Santa Clara.. } \\
\text { Contre Costa. }\end{array}$ & $\begin{array}{l}182 \\
162\end{array}$ \\
\hline Area 3-Sacrusnento Valley : & & Sonoma.... & 146 \\
\hline \multirow{2}{*}{ 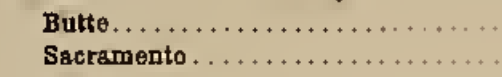 } & 116 & Others ".. & 379 \\
\hline & $\begin{array}{l}228 \\
900\end{array}$ & Additional counties to:al. . & 1,908 \\
\hline 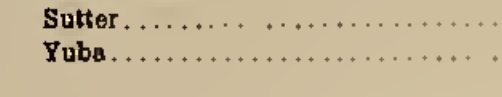 & 367 & State totall. & 25,120 \\
\hline Area total.... & 1,610 & & \\
\hline
\end{tabular}

Table 2 (Map 18)-Callifarnla Plums: Princlpal Varieties, Maturlty, Acreage, and Utllixatlon, 1953

\begin{tabular}{|c|c|c|c|c|}
\hline Tarietiles & 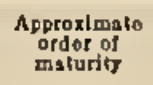 & Number ol scros & 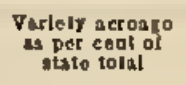 & Prtnclpal stilieanllon \\
\hline 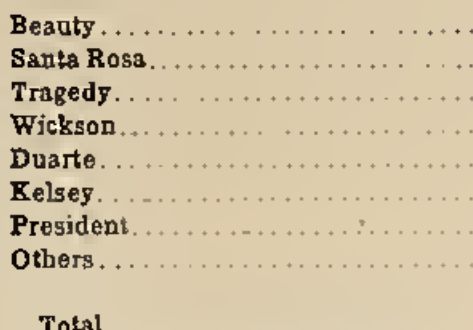 & $\begin{array}{c}1 \\
2 \\
3 \\
4 \\
6 \\
6 \\
6 \\
1-7\end{array}$ & $\begin{array}{r}2,641 \\
8,909 \\
798 \dagger \\
696 \\
3,813 \ddagger \\
699 \\
1,960 \\
6,704 \\
26,120\end{array}$ & $\begin{array}{r}10.1 \\
35.4 \\
3.2 \\
2.8 \\
16.2 \\
2.8 \\
7.8 \\
22.7 \\
-100.0\end{array}$ & $\begin{array}{l}\text { All varieties used for fresh market; } \theta \text { fe } \\
\text { are also sold for processing }\end{array}$ \\
\hline
\end{tabular}

\begin{tabular}{|c|c|c|c|c|c|c|c|c|c|c|c|}
\hline \multirow{2}{*}{ Varieties } & \multicolumn{5}{|c|}{ Por cent of area acreaso } & \multicolumn{6}{|c|}{ Per cenl ol atasto variety ocreago by areas } \\
\hline & Arou 1 & Area 2 & Aroa 3 & Aroa 4 & Aroa 5 & Area 1 & Area 2 & Area 3 & Area 4 & Aros $G$ & Totalal $3^{*}$ \\
\hline Beauty...... & 16 & 6 & 2 & & & 71 & 19 & 1 & & & 91 \\
\hline Santa Rosa $†$. & 63 & 11 & 26 & 28 & 47 & 68 & 10 & 6 & 3 & 6 & 91 \\
\hline Tragedy..... & 1 & 1 & 11 & 26 & 1 & 17 & 10 & 22 & 31 & 1 & 81 \\
\hline Wickson. & 3 & 3 & 1 & & & 60 & 39 & 3 & & 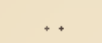 & 92 \\
\hline Duarte. & 6 & 34 & 11 & 12 & 1 & 17 & 73 & 4 & 3 &.. & 97 \\
\hline Belsey... & 2 & 6 & 2 & & & 26 & 67 & 5 & 1 & & 99 \\
\hline President. & 4 & 13 & 4 & 10 & 4 & 26 & 53 & 3 & 5 & 2 & 89 \\
\hline Others... & 15 & 26 & 43 & 26 & 47 & & & & . & & .. \\
\hline Total. & 100 & 100 & 100 & 100 & 100 & & & & & & \\
\hline
\end{tabular}




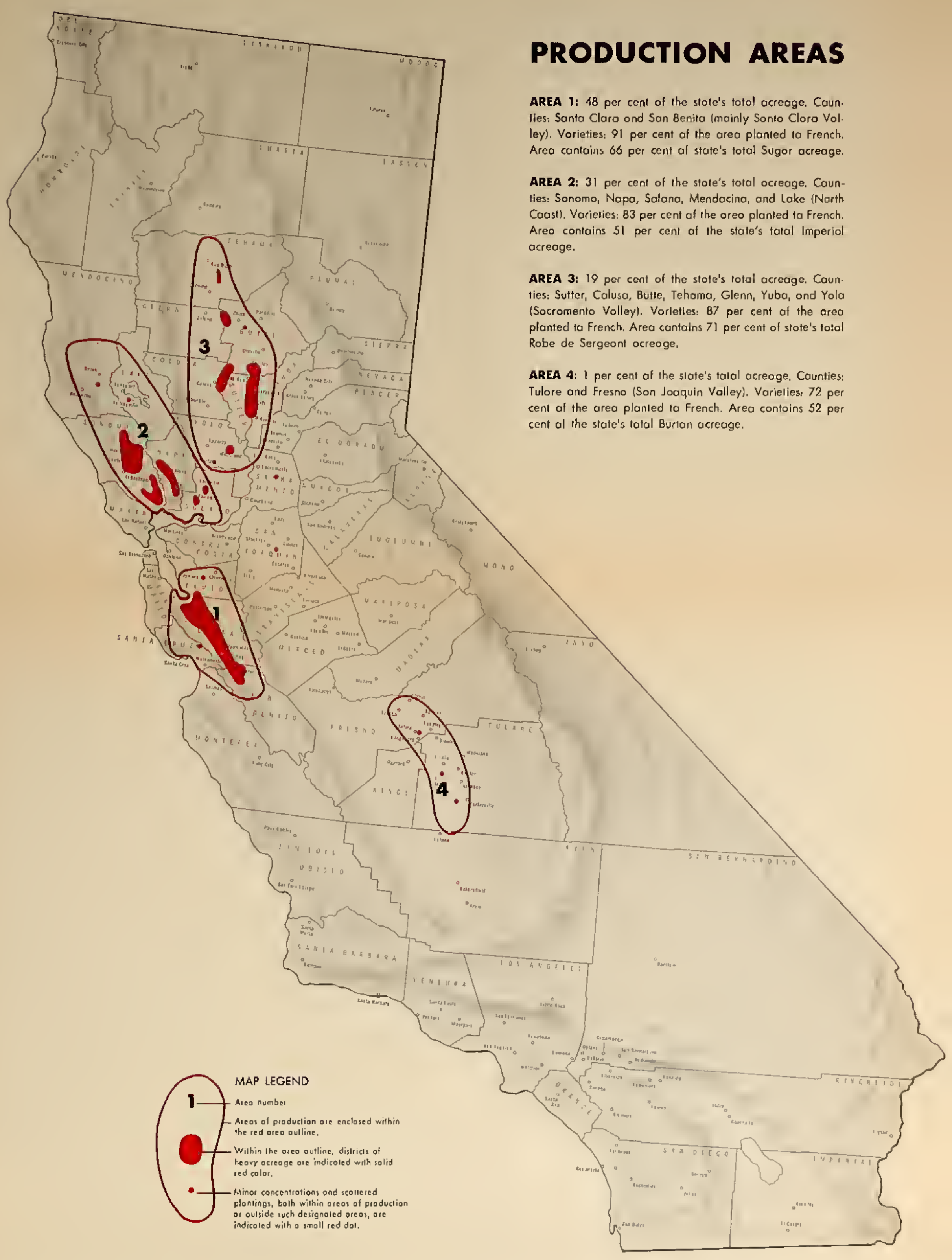

USUAL HARVEST SEASONS

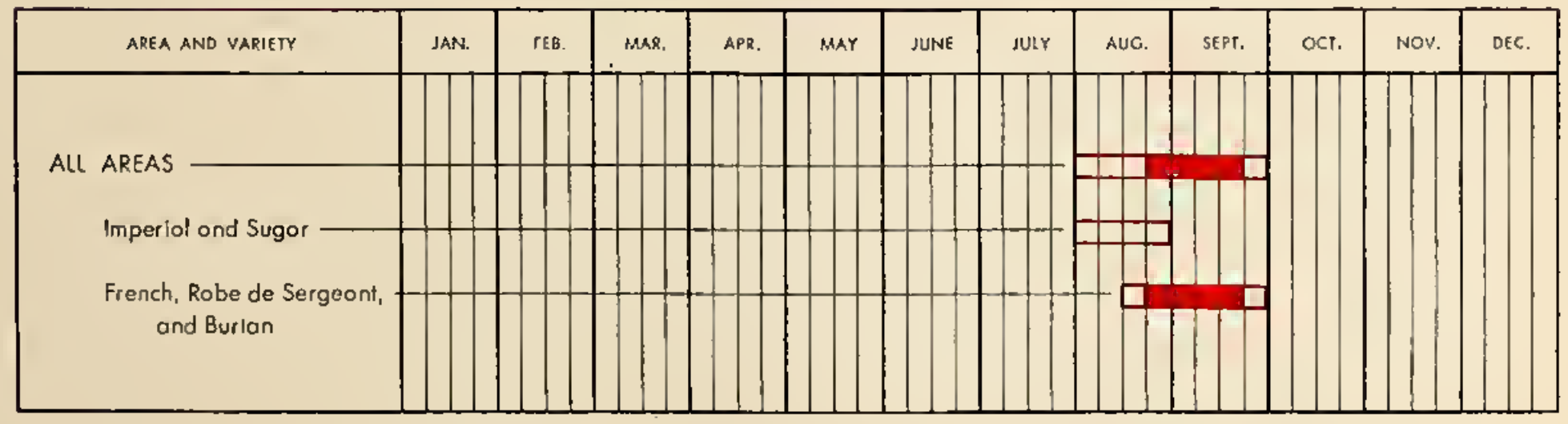

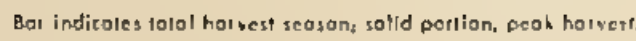




\begin{tabular}{|c|c|c|c|}
\hline \multicolumn{4}{|c|}{$\begin{array}{c}\text { Tabie } 1 \text { (Map 19)-Callfornia Prunes: Tatal Acreage (Boaring and Nanbearing) } \\
\text { by Areas and Cauntios, } 1953\end{array}$} \\
\hline Arez and coanty & Namber of aeres & Area and county & Namber of acres \\
\hline $\begin{array}{l}\text { Ares 1: } \\
\text { Alamed }\end{array}$ & & $\begin{array}{l}\text { Area 3-continued: } \\
\text { Sutter }\end{array}$ & \\
\hline San Benito.. & 3,249 & & $\begin{array}{l}6,111 \\
2,353\end{array}$ \\
\hline Santz Clars & 43,940 & Yolo..... & 1,079 \\
\hline Santa Cruz.. & 670 & Yubs.. & 1,201 \\
\hline Area total.. & 47,972 & Area total. & 19,075 \\
\hline Area 2: & & Area 4: & \\
\hline $\begin{array}{l}\text { Lalke. . . . } \\
\text { Mendocino }\end{array}$ & $\begin{array}{l}723 \\
825\end{array}$ & $\begin{array}{l}\text { Fresno.... } \\
\text { Tulare..... }\end{array}$ & $\begin{array}{l}313 \\
730\end{array}$ \\
\hline Napa.......... & 8,565 & & \\
\hline \multirow{2}{*}{ Sonoma. } & 4,192 & Areas total. . & 1,043 \\
\hline & 17,256 & & \\
\hline \multirow[t]{2}{*}{ Ares total. . } & 31,561 & Sacramento....... & 431 \\
\hline & & San Josquin....... & 239 \\
\hline \multirow{3}{*}{$\begin{array}{c}\text { Ares 3: } \\
\text { Butte... } \\
\text { Colusa... } \\
\text { Glean .. }\end{array}$} & & Others ${ }^{\circ} \ldots$ & 684 \\
\hline & $\begin{array}{l}3,091 \\
3,600\end{array}$ & Additional counties total. & 1,354 \\
\hline & 1,640 & State total. . & 101,005 \\
\hline
\end{tabular}

\begin{tabular}{|c|c|c|c|c|}
\hline \multicolumn{5}{|c|}{$\begin{array}{c}\text { Table } 2 \text { (Map 19)-Califarnia Prunes: Principai Varieties, Maturity, Acreage, } \\
\text { and Utllizatian, } 1953\end{array}$} \\
\hline Varieties & 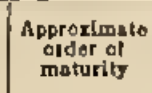 & Sumber of atres & 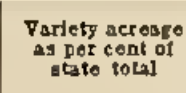 & Princtpal culflestion \\
\hline 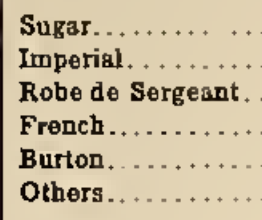 & $\begin{array}{r}1 \\
2 \\
3 \\
4 \\
4 \\
1-4\end{array}$ & $\begin{array}{r}3,382 \\
7,006 \\
1,325 \\
88,419 \\
240 \\
633\end{array}$ & $\begin{array}{r}3.4 \\
6.9 \\
1.3 \\
87.6 \\
0.2 \\
0.6\end{array}$ & $\begin{array}{l}\text { Dried, some for fresh marke } \\
\text { Dried } \\
\text { Dried } \\
\text { Dried } \\
\text { Dried }\end{array}$ \\
\hline Total. & & 101,005 & 100.0 & \\
\hline
\end{tabular}

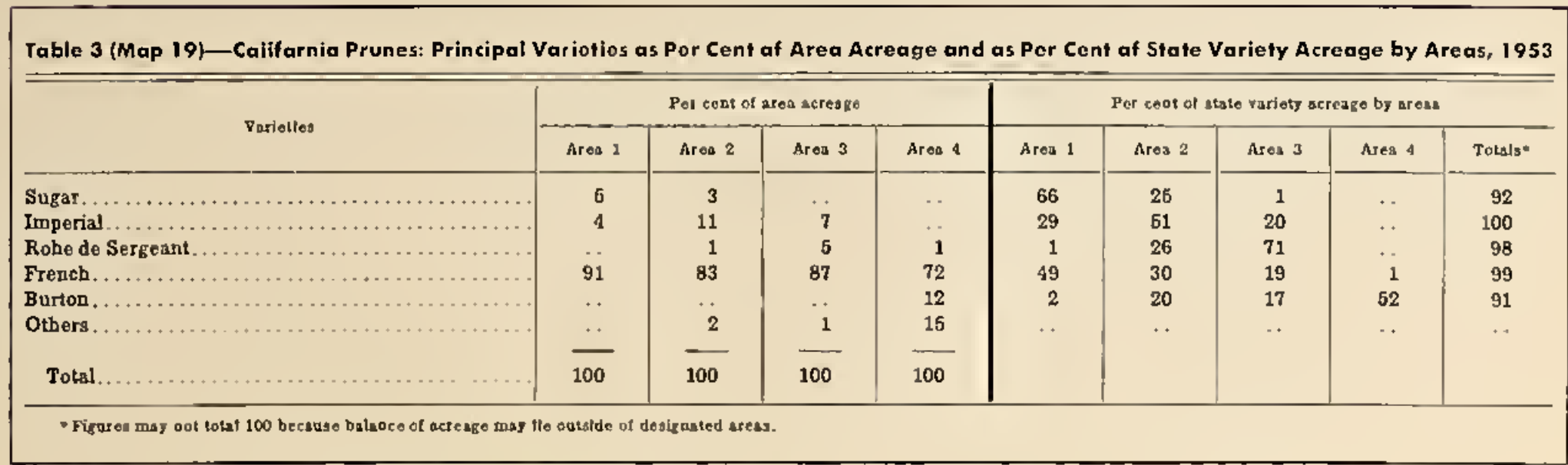

Saurces af data: Acreage estimates in Tables 1 and 2 are based an 1953 latal acreage estimates (bearing and nanbear. ing) made by the Califarnia Crap and Livestack Reparting Service, Sacramenta. Table 3 was campiled fram unpub- 


\section{WALNUTS}

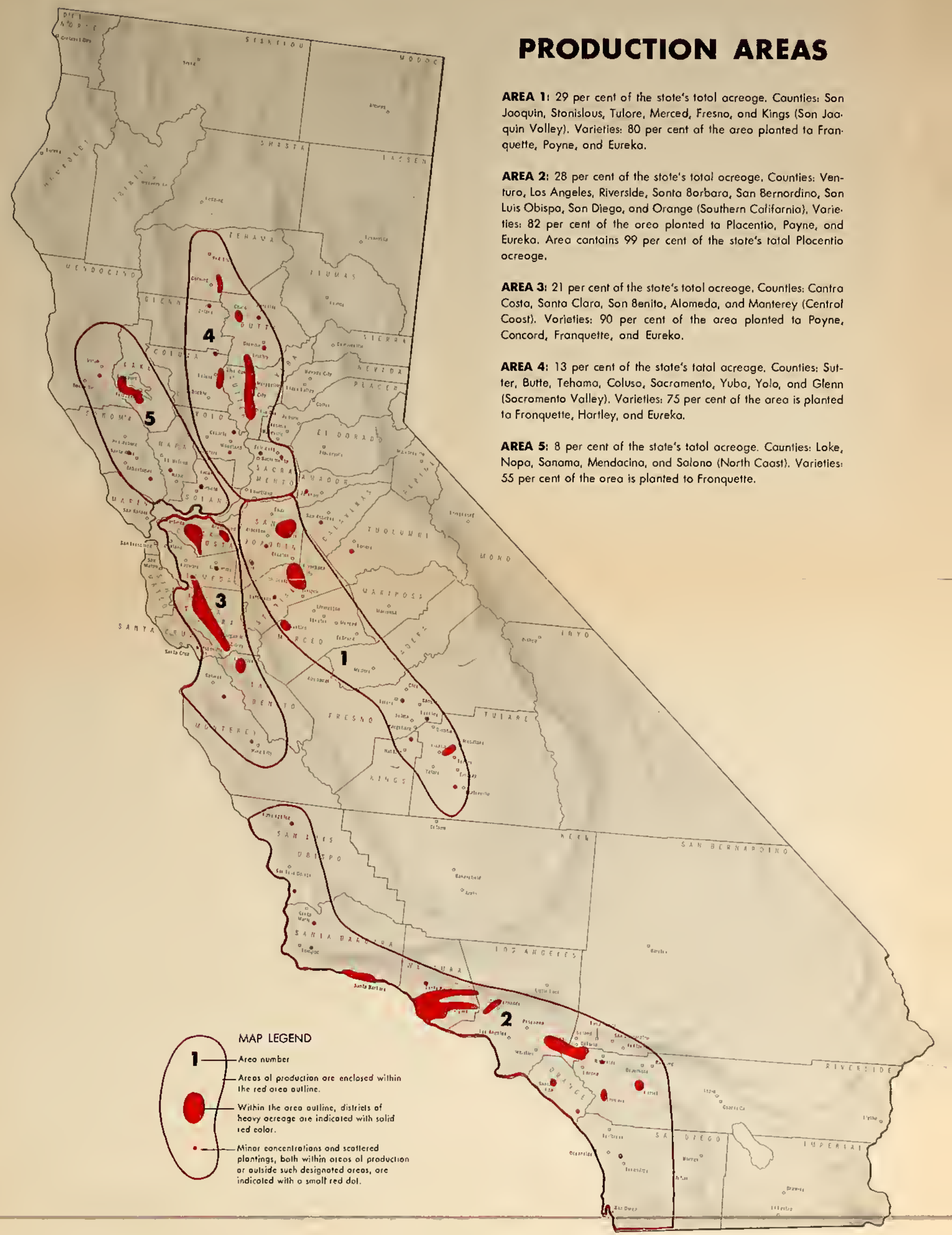

\section{USUAL HARVEST SEASONS}

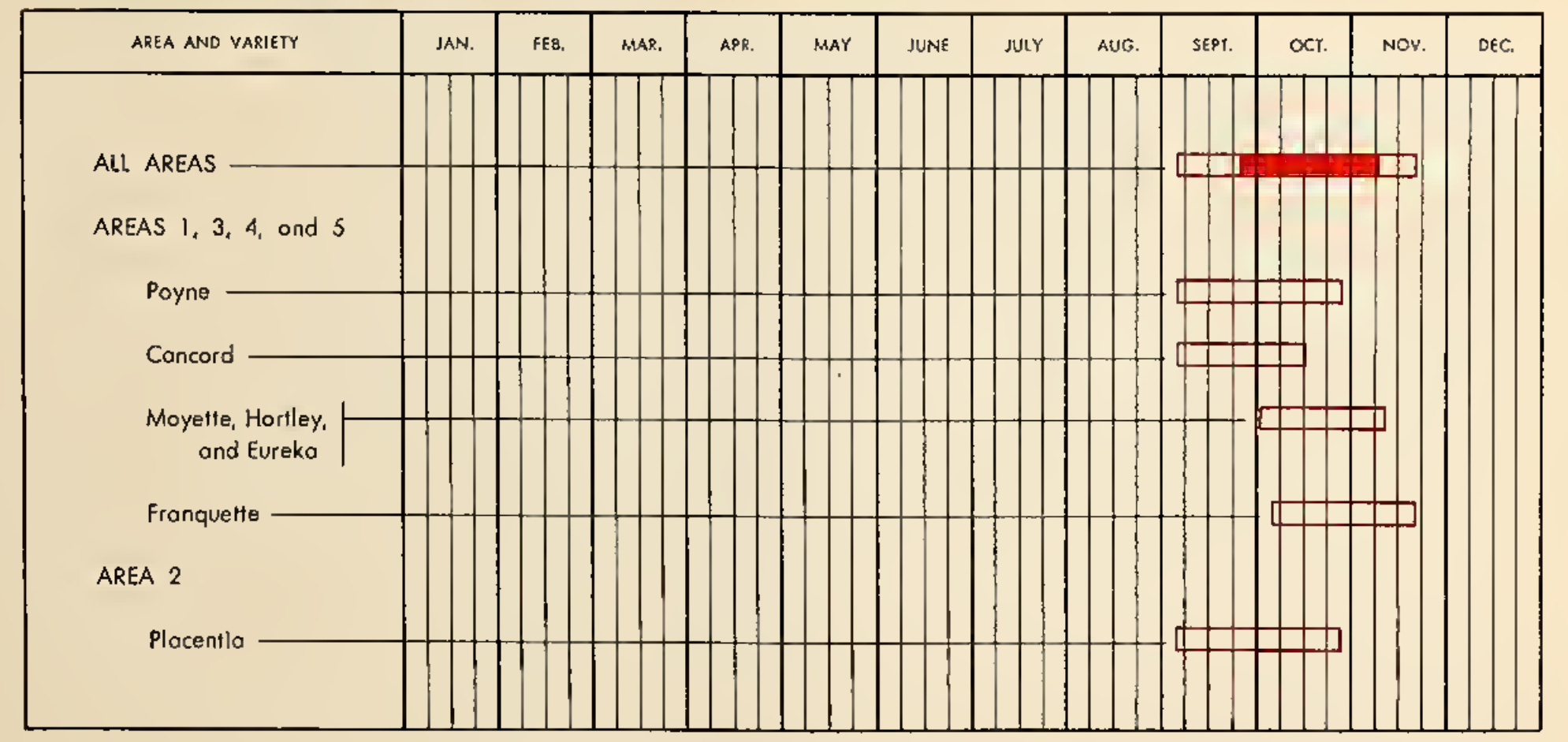




\begin{tabular}{|c|c|c|c|}
\hline \multicolumn{4}{|c|}{$\begin{array}{c}\text { Toblo } 1 \text { (Mop 20)—California Wolnuts: Jatal Acreage (Beoring and Nonbearing) } \\
\text { by Areas and Countios, } 1953\end{array}$} \\
\hline Aroes sod connty & Nutuber of acros & Area and county & Number of acres \\
\hline Area 1-San Joaquin Valley: & & Area 4-Sacramento Valley: & \\
\hline & 1,880 & & 3,676 \\
\hline Kings.... & 912 & Colusa. & 1,407 \\
\hline Madera. & 41 & Glenл..... & 852 \\
\hline & $\begin{array}{r}3,111 \\
13506\end{array}$ & Sscramento. & 1,381 \\
\hline San Josquin. & 13,586 & Sutter...... & 6,118 \\
\hline $\begin{array}{l}\text { Stanislaus... } \\
\text { Tutare. }\end{array}$ & 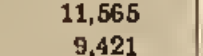 & Tehama. & 2,109 \\
\hline \multirow[b]{2}{*}{ Area total. . } & & Yubs... & $\begin{array}{r}878 \\
1,038\end{array}$ \\
\hline & 40,516 & & \multirow{2}{*}{17,459} \\
\hline Area 2-Southern California: & & Aró & \\
\hline Los Angeles ............... & 6,650 & Area 5-North Cosst: & \\
\hline Orange................... & 968 & Lake............. & 0,095 \\
\hline Riverside......... & 5,436 & Mendoçino. & 670 \\
\hline San Bernardino. & 2,591 & Napa. ...... & 1,992 \\
\hline San Diego....... & 1,001 & Solano.. & 627 \\
\hline San Lusis Obispo. & 1,039 & Sonoma. & 1,716 \\
\hline Sents Barbara... & 2,909 & & \\
\hline Vontura....... & 17,968 & Ares total.... & 11,100 \\
\hline \multirow[t]{2}{*}{ Ares total. . } & \multirow[t]{3}{*}{38,562} & Additional counties : & \\
\hline & & Calaveras.......... & 454 \\
\hline Ares 3-Central Coast: & & Amador... & 230 \\
\hline Alameda............. & 1,212 & El Dorado. & 162 \\
\hline Contra costn & $\begin{array}{r}14,963 \\
1,17\end{array}$ & Otbers ${ }^{\circ} \ldots$ & 580 \\
\hline \multirow{2}{*}{$\begin{array}{l}\text { San Benito... } \\
\text { Santa Clara.. }\end{array}$} & $\begin{array}{l}1,117 \\
2,071\end{array}$ & Additional counties total. & 1,426 \\
\hline & 9,188 & \multirow{2}{*}{ State total. . } & \multirow{2}{*}{137,614} \\
\hline Area total. . & 28,551 & & \\
\hline
\end{tabular}

\begin{tabular}{|c|c|c|c|c|}
\hline \multicolumn{5}{|c|}{ Toble 2 (Mop 20)—California Walnuts: Principal Varietles, Maturlty, Acreoge, } \\
ond Utllization, 1953
\end{tabular}

\begin{tabular}{|c|c|c|c|c|c|c|c|c|c|c|c|}
\hline \multicolumn{8}{|c|}{ Toble 3 (Map 20)—Colifornio Walnuts: Principal Variefies os Per Cent of Area Acreago and os Per Cent of } \\
State Variety Acreage by Areas, 1953 \\
\hline \hline
\end{tabular}

Sources of doio: Acreoge estimoies in Tobles 1 and 2 are bosed on 1953 folol ocreoge estimoies tbeoring ond nonbear ing) mode by the Colifornio Crop and livestock Reporting Service, Sactamento. Table 3 was compiled from unpub- 